\title{
Runtime revision of sanctions in normative multi-agent systems
}

\author{
Davide Dell'Anna $^{1}$ (D) Mehdi Dastani $^{1} \cdot$ Fabiano Dalpiaz $^{1}$
}

Published online: 16 June 2020

(c) The Author(s) 2020

\begin{abstract}
To achieve system-level properties of a multiagent system, the behavior of individual agents should be controlled and coordinated. One way to control agents without limiting their autonomy is to enforce norms by means of sanctions. The dynamicity and unpredictability of the agents' interactions in uncertain environments, however, make it hard for designers to specify norms that will guarantee the achievement of the system-level objectives in every operating context. In this paper, we propose a runtime mechanism for the automated revision of norms by altering their sanctions. We use a Bayesian Network to learn, from system execution data, the relationship between the obedience/violation of the norms and the achievement of the system-level objectives. By combining the knowledge acquired at runtime with an estimation of the preferences of rational agents, we devise heuristic strategies that automatically revise the sanctions of the enforced norms. We evaluate our heuristics using a traffic simulator and we show that our mechanism is able to quickly identify optimal revisions of the initially enforced norms.
\end{abstract}

Keywords Multiagent systems $\cdot$ Norm revision $\cdot$ Norm enforcement

\section{Introduction}

Multiagent systems (MASs) comprise autonomous agents that interact in a shared environment [57]. To achieve the system-level objectives of a MAS, the behavior of the autonomous agents should be controlled and coordinated [11]. For example, a smart traffic system is a MAS that includes autonomous agents like cars, traffic lights, etc. The objectives of the system include avoiding the occurrence of traffic jams as well as minimizing the number of accidents.

Davide Dell'Anna

d.dellanna@uu.nl

Mehdi Dastani

m.m.dastani@uu.nl

Fabiano Dalpiaz

f.dalpiaz@uu.nl

1 Utrecht University, Utrecht, The Netherlands 
One way to control the behavior of the agents in a MAS without limiting their autonomy is norm enforcement [1,47]. Norm enforcement via sanctions is traditionally contrasted with norm regimentation; the latter alternative prevents the agents from reaching certain states of affairs. For example, in a smart traffic system, a regimentation strategy is to close a road to prevent cars from entering that road, while a sanctioning strategy is to impose sanctions on cars that drive through the road.

Due to the dynamicity and unpredictability of the behaviours of interacting agents in uncertain environments, it is difficult for the designers who engineer a MAS to specify norms that, when enforced, will guarantee the achievement of system-level objectives in every operating context. To cope with this issue, the enforced norms need to be revised at runtime. Existing research has investigated the offline revision of the enforced norms [3], proposed logics that support norm change [4, 33, 34], and examined the legal effects of norm change [28].

In [23], we proposed a framework for engineering normative MASs that, using observed data from MAS execution, revises the norms in the MAS at runtime to maximize the achievement of the system objectives. In that work, we made the simplistic assumption that norms are regimented and we introduced algorithms for switching among alternative predefined norms. In [24] we extended the framework to support the revision of norm enforced via sanctioning. In addition to observed data from MAS execution, we used an estimation of the preferences of the agents to guide the runtime norm revision. However, we considered MASs where only one norm at a time was enforced.

In this paper, we significantly extend our previous work by supporting MASs where multiple norms are enforced. We formalize different types of rational agents that behave according to their preferences and we discuss their properties. We use Bayesian Networks to learn the norm effectiveness from data observed from MAS execution and to inform the runtime norm revision mechanism that revises the sanctions of multiple norms.

The contributions of this paper are as follows:

- We provide a formal definition of different types of rational preferences of agents, specified in terms of desired states of affairs and the maximum payment that the agent is willing to make to achieve such states of affairs. We prove that such preferences satisfy the basic rationality requirements [37].

- We build on and extend the general architecture proposed in [23, 24], and study in detail the relationships between estimated agents' preferences, sanctions, and systemlevel objectives. We use a framework where the normative MAS is flanked by a norm monitoring and enforcement component, and we introduce a norm revision component that uses observed data from MAS execution and an estimation of agents' preferences to modify norm sanctions at runtime.

- We propose six heuristic strategies for the revision of multiple norms that leverage probabilistic information learned from observed data from MAS execution and an estimation of the preferences of agents.

- We report on an evaluation through a traffic simulator that shows the effectiveness and efficiency of our revision strategies in identifying optimal sanctions for multiple norms.

Organization Section 2 reports on related work. Section 3 presents our framework to characterize norms and agents' preferences. Section 4 explains the overall approach for the supervision of normative MAS based on probabilistic reasoning over norm effectiveness and agents' preferences. Section 5 introduces six strategies for revising norms by combining agents' preferences with the achievement of the system-level objectives. Section 6 
evaluates our work through simulation experiments. Section 7 discusses the results and the assumptions, limitations and future directions of our work. Section 8 presents our conclusions.

\section{Related work}

In the MAS literature, norms have been proposed as a way to regulate the behavior of the agents in order to achieve system-level properties without limiting the autonomy of the agents $[1,47,52]$.

Many approaches focus on the design-time construction of robust normative MASs. Several techniques enable proving the correctness of normative systems through the model checking of formulas that describe liveness or safety properties [2, 22, 32]. These works are useful for the initial design of a MAS, but they cannot cope with the runtime unpredictability of the system that stems from the autonomy and heterogeneity of the agents.

In order to successfully supervise and regulate dynamic MASs, researchers have studied the revision of norms. Some frameworks formalize norm dynamics thereby allowing the assessment of the impact of norms on the specification of a MAS, i.e., whether the designed MAS will be norm compliant. Aucher et al. [4] introduce a dynamic context logic to describe the operations of contraction and expansion of theories that occur when removing or adding new norms. Governatori et al. [28] investigate how the application of theory revision leads to legal abrogations and annulments. Knobbout et al. [34] propose a dynamic logic to characterize the dynamics of state-based and action-based norms. Both in Knobbout's work [33,34] and in Alechina et al.'s approach [2], norm change is restricted to norm addition. This family of approaches focus on the impact of revising a norm on an existing normative system. In this paper, instead we study the relationship at runtime between the enforced norms and the achievement of system-level objectives, and suggest mechanisms to determine how to revise the (sanctions of the) current norms.

Jiang et al. [30] discuss the contextualization of norms. They explicitly represent the context of application of a norm and they use such context to organize norms during the design of a MAS. In our work, we also enforce different norms in different contexts. Unlike them, however, we determine the most appropriate context for different norm sets at runtime and based on observed data from MAS execution.

Miralles et al. [38] present a framework for the adaptation of MAS regulations at runtime. Their approach is complementary to ours. They represent conditional norms via norm patterns and describe an adaptation mechanism based on case-based reasoning. Adaptation is performed at runtime individually by a number of assistant agents and then, via a voting mechanism, a final adaptation is approved. The decision on how to adapt norms is taken based on similar previously seen cases. In their work, however, they do not consider sanctions. In our work, we focus on the revision of sanctions, we perform norm revision through a centralized component, and we make use of an estimation of agents' preferences to guide norm revision.

Cardoso et al. [12] present a framework for the runtime adaptation of sanctions associated with obligations. In their work, they assume that norm violations are bad for the system-level objectives. In our work, we relax such assumption, as agents ability to violate norms can be useful [13]. We evaluate the effectiveness of a norm at runtime based on observed data from MAS execution. Furthermore, they assume that the strength of a sanction should be directly proportional to its application frequency, and they constantly try 
to lower sanctions in order to give agents maximum autonomy. In our work, we base the revision of norms on an estimation of the preferences of the agents, and we determine the appropriate value of their sanctions based on the relationship between obedience of norms and achievement of system-level objectives determined at runtime.

In MASs, agents' preferences have been mainly used as a way to choose at runtime between different plans or actions to execute [20, 31, 41, 53]. Preferences are usually interpreted as constraints that, if satisfied by a certain plan (or action), increase the desirability of executing such plan (or action). Formal languages have been proposed and used for expressing preferences (e.g., $\mathcal{L P \mathcal { P }}[6,9]$ or $L T L$ [11]). In this work we focus on strategies for sanctions' revision. For this reason, we make use of a high-level representation of preferences, without restricting ourselves to, but supporting, any specific language. In particular, we consider preferences that satisfy the basic rationality requirements [37] and order different alternative states of affairs that agents may desire to achieve. Our agents are rational and norm-aware [50], in the sense that they always try to aim at the most preferred state of affairs for which they have enough budget, taking also into account the possible sanctions they would incur when violating some of the enforced norms. Furthermore, our agents are autonomous, in the sense that they are able to make decisions without the intervention of human users but in line with their preferences $[5,21]$. As we aim to investigate the process of norm revision, we assume that we have an accurate estimation of the agents' preferences. In future work, we can relax this assumption and investigate norm revision based on inaccurate estimations of the agents' preferences.

Chopra et al. [16] study how agents' preferences-expressed in terms of goals-interact with norms - represented as commitments. In particular, they propose a framework for the agents to adapt their behavior. We take an orthogonal approach, for we study how to change the norms without altering the agent construction. In particular, we study how to alter the sanctions used to enforce the norms on the agents, so to guarantee at runtime the system-level objectives. Our proposed mechanisms, therefore, relate also to the idea of adjustable autonomy [39]. The proposed runtime mechanism of revision of the sanctions of the norms can be seen as an automated mechanism to adjust the decisions' options of the agents (thus their degree of autonomy) so to maximize the objectives of the system and its operators.

Cranefield et al. [18] present a Bayesian approach to norm identification. They show that agents can internalize norms that exist in an environment, by learning from the behavior that complies with or violates certain norms. This work is a valuable addition to ours, for it shows that it is possible for agents to learn norms even when they are not explicitly communicated to them.

Tumer et al. [48] use multi-agent reinforcement learning in a smart traffic simulation to determine the behavior of the car agents that maximizes the utility of the city designer and of the individual agents. Their interesting work focuses on regimentation; instead, we focus on enforcement that does not violate agents' autonomy.

\section{Normative multiagent systems}

This section presents a generic framework for specifying normative multiagent systems in which the agents behave in line with their preferences while norms are enforced on them via sanctions. This framework allows us to analyze the interplay between norms and agents' preferences in normative multiagent systems. 
Fig. 1 Two lanes ring road. Rectangles are vehicles, moving in counter clockwise direction

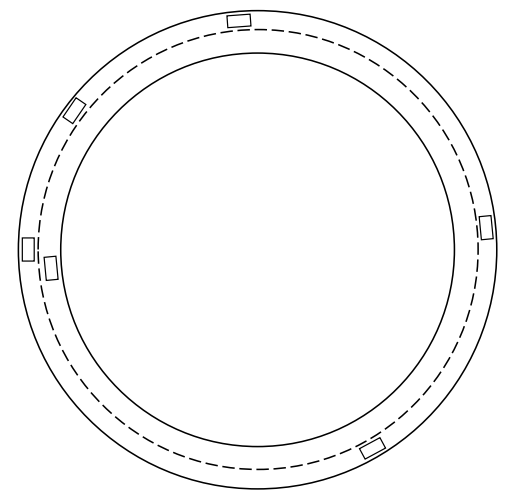

\subsection{Illustrative example}

Consider the two-lanes ring road depicted in Fig. 1. In a ring road, a population of vehicles moves continuously in a circle. Every vehicle is autonomous and acts according to its own preferences. For example, vehicles have preferences about, among other things, their speed, based on which they determine their willingness to risk sanctions for violating traffic norms. Such preferences and their corresponding willingness to risk sanctions allow the vehicles to autonomously decide when and how to accelerate or decelerate or to change lane. If a fast vehicle is using the outer line and a slower vehicle blocks its way, the fast vehicle may move to the inner line to overtake the slow vehicle. Since all vehicles share the same environment, their local decisions have an effect on the (emergent) system-level behavior of the vehicles driving on the ring road [46]. For example, based on contextual factors such as the density of vehicles on the ring road, the vehicles' behavior may provoke traffic jams and the average speed may vary, as well as the average time to complete a loop of the ring road. The ring road is a simple example of a MAS. Although far from realistic traffic situations, the ring road illustrates the fundamental phenomena of emergent systemlevel properties, caused by the local decisions of individual agents, and the importance of mechanisms to control and steer such system-level behaviors.

We assume that the main stakeholder of the ring road (the city council) has two systemlevel objectives: to minimize the average time to complete a loop of the ring road and to minimize the number of halted cars. Despite interdependence, the stakeholder desires to evaluate the two objectives independently due to their distinct nature. We consider two contextual variables that may influence the achievement of the system-level objectives, together with the vehicles' behavior: the density of vehicles and the presence of an obstacle on the ring road. The higher is the density of the vehicles on the ring road, the higher is the risk of breaking waves and slowdowns. The presence of an obstacle may force vehicles to halt and wait for an adequate moment to take over the obstacle. If the density of vehicles on the ring road is high enough, this may also cause queues after the obstacle. To achieve the objectives, the behavior of the agents is regulated by enforcing norms concerning (i) the speed limit, such as the norm every vehicle on the ring road shall not exceed a speed of $50 \mathrm{~km} / \mathrm{h}$, otherwise it will receive a sanction of $100 €$, and (ii) the minimum safety distance between cars, such as the norm every vehicle on the ring road shall keep a minimum distance of $2 m$, otherwise it will receive a sanction of $20 €$. Regulating speed and safety distance of the cars on the ring road is expected to help achieving the system-level objectives 
in the traffic contexts represented by the contextual variables. A car that keeps a sufficient safety distance from the car ahead, is less likely affected by sudden deceleration of the car ahead. More space between cars may also favour surpasses of slow cars when necessary. An opportune safety distance, together with opportune cars speed, may reduce jams in the presence of obstacles or the effect of breaking waves.

The ring road above described is a normative MAS. Vehicles are autonomous agents, each acting according to their own preferences. Each agent belongs to an agent type that can be characterized by the agent's preferences. For instance a cautious agent is a type of agent that prefers to go slow rather than fast on the ring road and prefers to maintain the appropriate safety distance. A brave agent is a type of agent that prefers to go fast rather than slow, and to approach cars closer than the minimum safety distance, even if it has to pay some money to do so.

\subsection{Norms}

The focus of this paper is the runtime revision of the sanctions of the norms enforced in the MAS. In order to focus on this aspect, we propose a simple but extensible language for norms. Consider a set of propositional atoms $L=\left\{p_{1}, \ldots, p_{k}\right\}$, each representing a fact that can hold or not in a system state ${ }^{1}$ (e.g., propositional atom $s p_{100}$ indicates that the speed of a vehicle on the ring road is $\leqslant 100 \mathrm{~km} / \mathrm{h}$ ).

Let $A L=\left(L_{1}, \ldots, L_{n}\right)$ be an ordered list of $n$ disjoint subsets of $L$, s.t. $L_{i}$ contains atoms related to an aspect $i$ of the system ${ }^{2}$ (e.g., $L_{i}=\left\{s p_{100}, s p_{50}\right\}$, in the ring road scenario, contains atoms related to the speed of the cars).

We consider a norm as a pair $N=(p, s)$, where $p \in L$ and $s \in \mathbb{N}$, indicating that $p$ should hold in the current system state for all agents, otherwise sanction $s$ will incur. For instance, a norm $N=\left(s p_{50}, 100\right)$ indicates that every vehicle on the ring road shall not exceed a speed of $50 \mathrm{~km} / \mathrm{h}$, otherwise it will receive a sanction of $100 €$.

In the following we consider an ordered set of norms $\mathcal{N}=\left\langle N_{1}, N_{2}, \ldots, N_{n}\right\rangle$ and assume that (i) norms are non-conflicting, i.e., obeying a norm $N_{i}$ does not prevent an agent from obeying or violating any other norm in $\mathcal{N}$; and (ii) each norm regulates a different aspect of the system, so that the $i$-th norm $N_{i}=(p, s)$ in $\mathcal{N}$ is a pair where $p \in L_{i}$ (with $L_{i} i$-th set in $A L)$ and $s \in \mathbb{N}$. For instance, if $A L=\left(L_{1}, L_{2}\right), L_{1}=\left\{s p_{50}, s p_{100}\right\}$ and $L_{2}=\left\{\right.$ dist $_{1}$, dist $\left._{2}\right\}$, then $N_{1}=\left(s p_{50}, 100\right)$ is a norm concerning the speed limit and $N_{2}=\left(\right.$ dist $\left._{2}, 100\right)$ is a norm concerning the minimum safety distance.

Note that, despite these assumptions, norms can still influence each others by means of the behavior that they cause on the agents. For instance, if the density of vehicles on the ring road is high, in order to obey a norm concerning the minimum safety distance from the car ahead, an agent may need to decrease its speed, therefore obeying also a norm concerning the maximum speed limit. We distinguish, however, such influence from the concept of conflict, in the sense that the norm concerning the minimum safety distance does not prevent, a priori, an agent to either obey or violate the norm concerning the maximum speed limit, and vice-versa.

\footnotetext{
1 A system state is assumed to consist of the state of individual cars (e.g., speed and position of the cars) as well as the state of the environment (e.g., density of vehicles in the ring road).

2 We use the term aspect to indicate any particular characteristics of the behavior of the agents, such as the speed of the cars, that is both monitorable by an organization that enforces norms in the MAS, and over which agents have control.
} 


\subsection{Rational agents and their preferences}

In MASs, agents are often assumed to be autonomous and possibly heterogeneous. Moreover, it is common to assume that the internal states of the agents such as their beliefs, preferences, and decision making mechanisms are unknown or partly known to other agents or to the institutions that regulate their behaviour. In line with the theory of economic rationality [37], in this paper we consider rational agents that behave according to their rational preferences, which determine an ordering between different alternative states of affairs (simply alternatives in the following). A rational agent aims to achieve its most preferred states of affairs: when a rational agent believes it is possible to achieve a certain state of affairs $s$, the agent will never aim to achieve states of affairs that are less preferred than $s$. For example, a cautious agent that prefers to go slow on the ring road and maintain appropriate safety distance, may be less prone to surpass other cars or to change lane, and may exhibit more moderate acceleration or deceleration than less cautious agents. The behavior of such a cautious agent, however, can vary significantly, based on contextual conditions. For example, a sudden break from the car ahead may force also the cautious agent to brusquely decelerate.

In this work, we assume we have an estimation of the preferences of the agents concerning the $n$ different aspects of the system that we aim to regulate by a norm, as per Sect. 3.2. In the rest of the paper, when we refer to the preferences of the agents, we refer therefore to such an estimation of their preferences. We do not assume access to the agents' internals such as their beliefs or their preferences regarding other aspects of the system (e.g., information about fuel reserve or the preference on road types). Having an estimation of the preferences of the agents should not be seen as a violation of the autonomy of agents or access to their internals. Having some knowledge of agents' preferences is realistic in most MAS settings. For example, in some cooperative settings, agents may be requested to declare their true preferences prior entering the system and agents can autonomously decide whether to join or not, while in other settings the preference of agents can be learned from their behaviors [8]. Note that we do not focus on the process of preference elicitation, which is essential for deriving and formulating agents' preferences, but beyond the scope of this paper. Several techniques for the elicitation of preferences have been proposed in literature, including both automated methods and methods that directly involve the end-user (see for example [10, 15, 44]). Here, we rely on such techniques and we just assume that some relevant part of agents' preferences is already given or estimated.

We represent the alternatives over which the agents have preferences as lists of pairs such as $\left(\left\langle p_{1}, b_{1}\right\rangle, \ldots,\left\langle p_{n}, b_{n}\right\rangle\right)$, indicating that for a state of affairs where $p_{1}, \ldots$, and $p_{n}$ hold, the agent is willing to spend, if necessary, a budget $b_{1}$ to achieve $p_{1}$, a budget $b_{2}$ to achieve $p_{2}$, etc. We focus on finite preferences, therefore we constrain the budgets expressed in the alternatives to be member of a budget set $\mathcal{B} \subset \mathbb{N}$.

We denote by $\operatorname{Pref}(a)=(A, \geq)$ the preference of an agent $a \in A g$, where $A g=\left\{a_{1}, \ldots, a_{n}\right\}$ is a set of agents, $A$ is a set of alternatives defined as per Definition 1, and $\geq$ is a partial order on $A$. We write $x \geq y$ to denote the fact that the agent either prefers alternative $x$ to alternative $y$ or is indifferent between $x$ and $y$.

Definition 1 (Preference Alternatives) Let $A L=\left(L_{1}, \ldots, L_{n}\right)$ be a list as per Sect. 3.2. Given a set of budget lists $B L \subseteq \mathcal{B}^{n}$ (with $\mathcal{B}^{n}$ the $n$-ary Cartesian power of $\mathcal{B}$ ), the set of alternatives $A$ is the set $\left\{\left(\left\langle p_{1}, b_{1}\right\rangle, \ldots,\left\langle p_{n}, b_{n}\right\rangle\right) \mid p_{i} \in L_{i} \&\left(b_{1}, \ldots, b_{n}\right) \in B L\right\}$. 
Notation Before continuing, we provide here a summary of the notation that we will use in the rest of the paper in the context of preferences. Given a preference Pref $(a)=(A, \geq)$, an alternative $x=\left(\left\langle p_{1}, b_{1}\right\rangle, \ldots,\left\langle p_{n}, b_{n}\right\rangle\right) \in A$, and a set of budget lists $B L \subseteq \mathcal{B}^{n}$, we call:

- $\operatorname{prop}(x)=\left(p_{1}, \ldots, p_{n}\right)$, the list of propositional atoms in $x$

- $\operatorname{bud}(x)=\left(b_{1}, \ldots, b_{n}\right) \in B L$, the list of budgets associated to each propositional atom in $x$.

- $\quad r e q \_b u d(x)=\sum_{b \in b u d(x)} b$ the budget required by alternative $x$ (required budget, in the following), i.e., the sum of all budgets in $x$.

- $x\left[B^{\prime}\right]$ a new alternative $x^{\prime}=\left(\left\langle p_{1}, b_{1}^{\prime}\right\rangle, \ldots,\left\langle p_{n}, b_{n}^{\prime}\right\rangle\right)$ with same propositional atoms as $x$, but using budgets $B^{\prime}=\left(b_{1}^{\prime}, \ldots, b_{n}^{\prime}\right) \in B L$ instead of budgets $B=\left(b_{1}, \ldots, b_{n}\right)$.

Furthermore, in the rest of the paper, unless specified otherwise, when we provide an example concerning preferences or norms, we make use of $L$ defined as the set $\left\{s p_{50}, s p_{100}\right.$, dist $_{1}$, dist $\left._{2}\right\}$ with $A L=\left(L_{1}, L_{2}\right)$ and $L_{1}=\left\{s p_{50}, s p_{100}\right\}$ and $L_{2}=\left\{\right.$ dist $_{1}$, dist $\left._{2}\right\}$ so that $\mathcal{N}=\left\langle N_{1}, N_{2}\right\rangle$ with $L_{1}$ related to $N_{1}$ (norm concerning speed limit) and $L_{2}$ related to $N_{2}$ (norm concerning safety distance), and we use $n$ to indicate the number of norms in $\mathcal{N}$.

In the following we define the types of preferences that we consider in this paper. We first define two basic types of preferences. Then, after providing some examples of such preferences, we define more complex preferences that combine the two basic types.

\subsubsection{Basic preferences}

We define here two types of basic preferences. The first kind of preference orders the alternatives based on their budgets, while the second type orders the alternatives based on the propositional atoms (i.e., states).

Definition 2 (Basic Preference) Given a list $A L=\left(L_{1}, \ldots, L_{n}\right)$ and a set $B L \subseteq \mathcal{B}^{n}$, an agent is said to have a basic preference $(A, \succeq)$ when for all alternatives $x$ and $y$ in $A$, the partial order $\geq$ satisfies one of the following two clauses:

(a) $x \geq y$ iff

req_bud $(x) \leqslant$ req_bud $(y) \&$

$\forall v, w \in A, \forall B, B^{\prime} \in B L: v[B]>w[B] \Rightarrow v\left[B^{\prime}\right]>w\left[B^{\prime}\right]$

(b) $x \geq y$ iff

if $\operatorname{prop}(x)=\operatorname{prop}(y)$ then $r e q \_b u d(x) \leqslant \operatorname{req} \_b u d(y)$

else $\forall B, B^{\prime} \in B L: x[B] \geq y\left[B^{\prime}\right]$

In the rest of the paper, we write $x \sim y$ when $x \geq y$ and $y \geq x$. We write $x>y$ when $x \geq y$ but not $y \geq x$.

If an agent's preference adheres to Definition 2a, then the required budget determines the order of the alternatives. In particular, Definition 2 a determines a preference where alternatives that require a lower budget are preferred to alternatives that require higher budget (first condition of Definition 2a) and the relative order between two alternatives with different propositional atoms is the same for all possible budgets (second condition of Definition 2a). Note that in a basic preference that adheres to Definition 2a, two alternatives $x$ and $y$ such that $r e q \_b u d(x)>r e q \_b u d(y)$ cannot be equally preferred. In fact, if $x \sim y$ we have that req_bud $(x) \leqslant r e q \_b u d(y)$ and $r e q \_b u d(y) \leqslant r e q \_b u d(x)$. As a consequence, all alternatives 
with required budget 0 are strictly preferred to all the other alternatives, and all alternatives with same required budget are equally preferred.

If an agent's preference adheres to Definition $2 b$, then the propositional atoms determine the order of the pairs. If a set of propositional atoms is preferred to another, then it is preferred regardless of the required budget. In a preference that adheres to Definition $2 b$ though, the alternatives with required budget 0 are strictly preferred to all the other alternatives with same propositional atoms.

We would like to emphasize that the basic preferences as we defined here are different than lexicographic ordering [27]. An agent's preference, as per Definition 2, satisfies, instead, the basic rationality requirements [37], as per Proposition 1.

Proposition 1 A basic preference Pref $(a)=(A, \succeq)$ for an agent $a \in A g$ is

- transitive $\forall x, y, z \in A$ if $x \geq y$ and $y \geq z$ then $x \geq z$; and

- complete $\forall x, y \in A$ either $x \geq y$ or $y \geq x$ or $x \sim y$.

Proof See "Appendix 1".

\subsubsection{Examples of basic preferences}

Given $\mathcal{B}=\{0,1\}$ and $B L=\mathcal{B}^{2}$, an example of basic preference defined according to Definition $2 \mathrm{a}$ is the following.

$$
\begin{aligned}
& \left(\left\langle s p_{100}, 0\right\rangle,\left\langle\text { dist }_{1}, 0\right\rangle\right) \geq\left(\left\langle s p_{100}, 0\right\rangle,\left\langle\text { dist }_{2}, 0\right\rangle\right) \geq \\
& \left.\left(\left\langle s p_{50}, 0\right\rangle,\left\langle\text { dist }_{1}, 0\right\rangle\right) \geq\left(\left\langle s p_{50}, 0\right\rangle,\left\langle\text { dist }_{2}, 0\right\rangle\right)\right\rangle \\
& \left(\left\langle s p_{100}, 0\right\rangle,\left\langle\text { dist }_{1}, 1\right\rangle\right) \geq\left(\left\langle s p_{100}, 1\right\rangle,\left\langle\text { dist }_{1}, 0\right\rangle\right) \geq \\
& \left(\left\langle s p_{100}, 0\right\rangle,\left\langle\text { dist }_{2}, 1\right\rangle\right) \succeq\left(\left\langle s p_{100}, 1\right\rangle,\left\langle\text { dist }_{2}, 0\right\rangle\right) \succeq \\
& \left(\left\langle s p_{50}, 0\right\rangle,\left\langle\text { dist }_{1}, 1\right\rangle\right) \geq\left(\left\langle s p_{50}, 1\right\rangle,\left\langle\text { dist }_{1}, 0\right\rangle\right) \geq \\
& \left.\left(\left\langle s p_{50}, 0\right\rangle,\left\langle\text { dist }_{2}, 1\right\rangle\right) \geq\left(\left\langle s p_{50}, 1\right\rangle,\left\langle\text { dist }_{2}, 0\right\rangle\right)\right\rangle \\
& \left(\left\langle s p_{100}, 1\right\rangle,\left\langle\text { dist }_{1}, 1\right\rangle\right) \geq\left(\left\langle s p_{100}, 1\right\rangle,\left\langle\text { dist }_{2}, 1\right\rangle\right) \geq \\
& \left(\left\langle s p_{50}, 1\right\rangle,\left\langle\text { dist }_{1}, 1\right\rangle\right) \geq\left(\left\langle s p_{50}, 1\right\rangle,\left\langle\text { dist }_{2}, 1\right\rangle\right)
\end{aligned}
$$

Note that in preference (1), alternatives with lower required budget are preferred over alternatives with higher required budget and the agents' prefers $s p_{100}$ over $s p_{50}$ for every safety distance, and dist $t_{1}$ over dist $_{2}$ for every speed.

Given $\mathcal{B}=\{0,1\}$ and $B L=\mathcal{B}^{2}$, an example of basic preference defined according to Definition $2 b$ is the following. 


$$
\begin{aligned}
& \left(\left\langle s p_{100}, 0\right\rangle,\left\langle\text { dist }_{1}, 0\right\rangle\right) \succ\left(\left\langle s p_{100}, 0\right\rangle,\left\langle\text { dist }_{1}, 1\right\rangle\right) \geq \\
& \left.\left(\left\langle s p_{100}, 1\right\rangle,\left\langle\text { dist }_{1}, 0\right\rangle\right) \succ\left(\left\langle s p_{100}, 1\right\rangle,\left\langle\text { dist }_{1}, 1\right\rangle\right)\right\rangle \\
& \left.\left(\left\langle s p_{50}, 0\right\rangle,\left\langle\text { dist }_{1}, 0\right\rangle\right)\right\rangle\left(\left\langle s p_{50}, 0\right\rangle,\left\langle\text { dist }_{1}, 1\right\rangle\right) \geq \\
& \left.\left.\left(\left\langle s p_{50}, 1\right\rangle,\left\langle\text { dist }_{1}, 0\right\rangle\right)\right\rangle\left(\left\langle s p_{50}, 1\right\rangle,\left\langle\text { dist }_{1}, 1\right\rangle\right)\right\rangle \\
& \left.\left(\left\langle s p_{100}, 0\right\rangle,\left\langle\text { dist }_{2}, 0\right\rangle\right)\right\rangle\left(\left\langle s p_{100}, 0\right\rangle,\left\langle\text { dist }_{2}, 1\right\rangle\right) \geq \\
& \left.\left.\left(\left\langle s p_{100}, 1\right\rangle,\left\langle\text { dist }_{2}, 0\right\rangle\right)\right\rangle\left(\left\langle s p_{100}, 1\right\rangle,\left\langle\text { dist }_{2}, 1\right\rangle\right)\right\rangle \\
& \left.\left(\left\langle s p_{50}, 0\right\rangle,\left\langle\text { dist }_{2}, 0\right\rangle\right)\right\rangle\left(\left\langle s p_{50}, 0\right\rangle,\left\langle\text { dist }_{2}, 1\right\rangle\right) \geq \\
& \left(\left\langle s p_{50}, 1\right\rangle,\left\langle\text { dist }_{2}, 0\right\rangle\right)>\left(\left\langle s p_{50}, 1\right\rangle,\left\langle\text { dist }_{2}, 1\right\rangle\right)
\end{aligned}
$$

Notice that in preference (2) states of affairs where $s p_{100}$ and dist hold are preferred over states of affairs where $s p_{50}$ and dist $t_{1}$ hold, regardless of the budget. Analogously, regardless of the budget, states of affairs where $s p_{50}$ and dist $t_{1}$ hold are preferred over states of affair where $s p_{100}$ and dist 2 hold, which, in turn, are preferred over states of affair where $s p_{50}$ and dist $_{2}$ hold. Such preference describes an agent type that prefers to drive fast rather than slow and that prefers to have a short safety distance rather than high, for whom maximizing speed and minimizing safety distance have priority over minimizing the budget to be spent, and, finally, who gives more importance to having a short safety distance rather than driving fast.

Finally, an example of a preference that does not satisfy Definition 2 is $\left(\left\langle s p_{50}, 1\right\rangle,\left\langle\right.\right.$ dist $\left.\left.\left._{1}, 1\right\rangle\right)\right\rangle\left(\left\langle s p_{50}, 0\right\rangle,\left\langle\right.\right.$ dist $\left.\left._{1}, 0\right\rangle\right)>\cdots$. This is because the first two alternatives share the same propositional atoms but the alternative with higher required budget is preferred to the alternative with lower required budget.

\subsubsection{Preferences}

The basic preference as defined in Definition 2 may not be expressive enough to capture some realistic cases. In order to cover more cases and to make our approach applicable to model more realistic scenarios, we consider more complex types of agents' preferences that combines the two basic types of preferences (defined in Definitions $2 a$ and $2 b$ ).

Intuitively, a rational agent may exhibit different preferences when the required budget increases. For example, consider a brave agent that prefers to drive fast and to keep a short safety distance rather than long, e.g., as per preference (2). Suppose, however, that such an agent is ready to pay only up to $1 €$ for driving fast and for keeping short safety distance. In such a case, the agent would prefer to drive fast and to keep a short safety distance, compared to other alternatives (e.g., to drive slow and keep a long safety distance), if the required budget is lower than $1 €$. For example, in preference (2), ordered according to Definition $2 \mathrm{~b}$, we have $\left(\left\langle s p_{100}, 1\right\rangle,\left\langle\right.\right.$ dist $\left.\left.\left._{1}, 1\right\rangle\right)\right\rangle\left(\left\langle s p_{50}, 0\right\rangle,\left\langle\right.\right.$ dist $\left.\left._{1}, 0\right\rangle\right)$. If the required budget for either driving fast or keeping a short safety distance is higher than 1 , however, the agent may instead give priority to spending the least possible. For example, $\left(\left\langle s p_{50}, 0\right\rangle,\left\langle\right.\right.$ dist $\left.\left._{1}, 2\right\rangle\right)$, not reported in preference (2), would be preferred to $\left(\left\langle s p_{100}, 1\right\rangle,\left\langle d i s t_{1}, 2\right\rangle\right)$, adhering to Definition $2 \mathrm{a}$ instead of Definition $2 \mathrm{~b}$. In other words, a rational agent may use different criteria to order the alternatives in a preference depending on the required budget.

We formalize this intuition by defining a type of preference $(A, \geq)$ that is a sequence of $k$ basic preferences, with $1 \leqslant k \leqslant|\mathcal{B}|$. We call such a complex preference simply preference. Each of the $k$ basic preferences adhere to either Definition 2a or Definition 2b, and the alternatives in the different basic preferences have increasing budgets. In particular, the set of 
possible budget lists $B L_{i} \subseteq \mathcal{B}^{n}$ for an alternative in the $i$-th basic preference $\left(A_{i}, \geq_{i}\right)$, for $i \leqslant k$, is determined as per Definition 3.

Definition 3 (Budget Lists of the $i$-th Basic Preference) Consider a set $\mathcal{B} \subset \mathbb{N}$, and $k$ disjoint subsets of $\mathcal{B}$, i.e., $\mathcal{B}_{1}, \ldots, \mathcal{B}_{k}$, such that each element of $\mathcal{B}_{i}$ is bigger than each element of $\mathcal{B}_{j}$, for $j<i \leqslant k$. In a preference composed by $k$ basic preferences, the set of possible budget lists for the alternatives in the $i$-th basic preference $\left(A_{i}, \geq_{i}\right)$, for $i \leqslant k$, is $B L_{i}=\left(\bigcup_{j \leqslant i} \mathcal{B}_{j}\right)^{n} \backslash \bigcup_{j<i} B L_{j}$

For instance, given the set $\mathcal{B}=\{0,1,2\}$ and $k=2$ two possible subsets of $\mathcal{B}$ as per Definition 3 are $\mathcal{B}_{1}=\{0,1\}$ and $\mathcal{B}_{2}=\{2\}$. The possible budget lists for the alternatives of 2 basic preferences are therefore $B L_{1}=\{(0,0),(0,1),(1,0),(1,1)\}$ and $B L_{2}=\{(0,2),(1,2),(2,0),(2,1),(2,2)\}$. In other words, the budgets in the alternatives of the $i$-th basic preference are always lower or equal to $\max \left(\mathcal{B}_{i}\right)$. This means that the required budget of every alternative in $A_{i}$ is always lower or equal to $n \cdot \max \left(\mathcal{B}_{i}\right)$, while the required budget of every alternative in $A_{i+1}$ is always higher or equal to $n \cdot \max \left(\mathcal{B}_{i}\right)$.

Definition 4 (Preference) Let $\left(A_{1}, \geq_{1}\right), \ldots,\left(A_{k}, \geq_{k}\right)$ be $k$ basic preferences as per Definition 2, such that alternatives in $A_{i}$ are defined with respect to a set of budget lists $B L_{i}$ as per Definition 3. An agent is said to have a preference $(A, \geq)$, iff $A=\bigcup_{i=1}^{k} A_{i}$ and $\succeq=\bigcup_{i=1}^{k} \geq_{i} \cup\left\{(x, y) \mid x \in A_{j} \& y \in A_{i} \& 1 \leqslant j<i \leqslant k\right\}$.

Note that a preference $(A, \succeq)$ that is composed by only one basic preference $\left(A_{1}, \geq_{1}\right)$ so that $A=A_{1}$ for $B L_{1} \subseteq \mathcal{B}^{n}$, and $\geq=\geq_{1}$, is a basic preference. If a preference is composed by more than one basic preference, every basic preference $\left(A_{i}, \geq_{i}\right)$ composing the preference adheres to either Definition 2a or Definition $2 \mathrm{~b}$, and for every pair of alternatives $x, y \in A$ such that $x \in A_{i}, y \in A_{j}$ and $i<j$, it holds that req_bud $(x) \leqslant r e q \_b u d(y)$. Furthermore, notice that the sets $A_{1}, \ldots, A_{k}$ of alternatives of the $k$ basic preferences composing a preference $(A, \geq)$ are disjoint subsets of $A$, since the possible budget lists of the $k$ basic preferences are disjoint subsets of $\mathcal{B}^{n}$.

Again, we note that a preference as per Definition 4 is transitive and complete.

Proposition 2 A preference Pref $(a)=(A, \geq)$ for an agent $a \in A g$ is

- transitive $\forall x, y, z \in A$ if $x \geq y$ and $y \geq z$ then $x \geq z$; and

- complete $\forall x, y \in A$ either $x \geq y$ or $y \geq x$ or $x \sim y$.

Proof See “Appendix 1".

\subsubsection{Examples of preferences}

An example of a preference composed by two basic preferences $\left(A_{1}, \geq_{1}\right)$ and $\left(A_{2}, \geq_{2}\right)$ is given in Eq. (3), given $\mathcal{B}=\{0,1,2\}$. 
\#from here ordered by Definition $2 b$

$$
\begin{aligned}
& \left(\left\langle s p_{100}, 0\right\rangle,\left\langle\text { dist }_{1}, 0\right\rangle\right)>\left(\left\langle s p_{100}, 0\right\rangle,\left\langle\text { dist }_{1}, 1\right\rangle\right) \geq \\
& \left.\left(\left\langle s p_{100}, 1\right\rangle,\left\langle\text { dist }_{1}, 0\right\rangle\right)>\left(\left\langle s p_{100}, 1\right\rangle,\left\langle\text { dist }_{1}, 1\right\rangle\right)\right\rangle \\
& \left(\left\langle s p_{50}, 0\right\rangle,\left\langle\text { dist }_{1}, 0\right\rangle\right)>\cdots>\left(\left\langle s p_{50}, 1\right\rangle,\left\langle\text { dist }_{1}, 1\right\rangle\right)>\cdots> \\
& \left(\left\langle s p_{50}, 1\right\rangle,\left\langle\text { dist }_{2}, 1\right\rangle\right)>
\end{aligned}
$$

\#from here ordered by Definition $2 a$

$$
\begin{aligned}
& \left(\left\langle s p_{100}, 0\right\rangle,\left\langle\text { dist }_{1}, 2\right\rangle\right) \geq\left(\left\langle s p_{100}, 2\right\rangle,\left\langle\text { dist }_{1}, 0\right\rangle\right) \geq \cdots> \\
& \left(\left\langle s p_{100}, 2\right\rangle,\left\langle\text { dist }_{1}, 2\right\rangle\right)>\left(\left\langle s p_{50}, 0\right\rangle,\left\langle\text { dist }_{1}, 2\right\rangle\right) \geq \cdots \geq \\
& \left(\left\langle s p_{50}, 2\right\rangle,\left\langle\text { dist }_{2}, 2\right\rangle\right)
\end{aligned}
$$

In such preference, the budget lists of the alternatives in $A_{1}$ are elements of $B L_{1}=\{(0,0),(0,1),(1,0),(1,1)\}$ for $\mathcal{B}_{1}=\{0,1\}$, and the alternatives are ordered by Definition $2 \mathrm{~b}$. The budget lists of the alternatives in $A_{2}$, instead, are elements of $B L_{2}=\{(0,2),(1,2),(2,0),(2,1),(2,2)\}$, for $\mathcal{B}_{2}=\{2\}$, and they are ordered by Definition $2 \mathrm{a}$. The required budget of every alternative in $A_{1}$ is lower or equal to 2 , while the required budget of every alternative in $A_{2}$ is higher or equal to 2 and lower or equal to 4 .

\subsubsection{Consistent preferences}

The preferences above described allow to express a multitude of possible orderings between different states of affairs. In the following we define an additional property that a preference can exhibit. We call such property consistency [29].

Intuitively a preference is consistent if when a state of affairs where a propositional atom $p$ holds is preferred to a state of affair where $q$ holds, then states of affairs where $p$ holds are preferred to states of affairs where $q$ holds also when a third atom $r$ is considered. For instance, if $\left(\left\langle p, b_{1}\right\rangle,\left\langle x, b_{2}\right\rangle\right) \geq\left(\left\langle q, b_{1}\right\rangle,\left\langle x, b_{2}\right\rangle\right)$, then in a consistent preference this holds for every propositional atom $x$.

Notice that preferences as per Definition 4 are not necessarily consistent. An example of a preference that is not consistent (i.e., does no exhibit the consistency property) is the following:

$$
\begin{aligned}
& \left(\left\langle s p_{100}, 0\right\rangle,\left\langle\text { dist }_{1}, 0\right\rangle\right)>\left(\left\langle s p_{80}, 0\right\rangle,\left\langle\text { dist }_{1}, 0\right\rangle\right)>\left(\left\langle s p_{80}, 0\right\rangle,\left\langle\text { dist }_{2}, 0\right\rangle\right)> \\
& \left(\left\langle s p_{50}, 0\right\rangle,\left\langle\text { dist }_{1}, 0\right\rangle\right)>\left(\left\langle s p_{50}, 0\right\rangle,\left\langle\text { dist }_{2}, 0\right\rangle\right)>\left(\left\langle s p_{100}, 0\right\rangle,\left\langle\text { dist }_{2}, 0\right\rangle\right)>\cdots
\end{aligned}
$$

Notice that, given dist $1, s p_{100}$ is preferred to $s p_{80}$, but given $d i s t_{2}, s p_{80}$ is preferred to $s p_{100}$.

We define consistent preferences by means of an enumeration condition over the propositional atoms of the alternatives. In particular, if two alternatives $x$ and $y$ with same budget lists differ exactly for one propositional atom, then if $x$ is preferred to $y$, this has to hold also for all other pairs of alternatives with same budget lists differing exactly for the same propositional atoms as $x$ and $y$. Intuitively the enumeration condition imposes an ordering on the alternatives that corresponds to an ordering that can be obtained by systematically enumerating the possible combinations of propositional atoms. For instance, if, given dist ${ }_{1}$, the proposition $s p_{100}$ from the set $\left\{s p_{100}, s p_{50}\right\}$ is enumerated before proposition $s p_{50}$ (i.e, $\left(\left\langle\right.\right.$ dist $\left.\left.\left._{1}, b_{1}\right\rangle,\left\langle s p_{100}, b_{2}\right\rangle\right)\right\rangle\left(\left\langle\right.\right.$ dist $\left.\left.\left._{1}, b_{1}\right\rangle,\left\langle s p_{50}, b_{2}\right\rangle\right)\right)$, then in a consistent preference $s p_{100}$ is enumerated before $s p_{50}$ also given dist $_{2}\left(\right.$ i.e, $\left(\left\langle\right.\right.$ dist $\left.\left.\left._{2}, b_{1}\right\rangle,\left\langle s p_{100}, b_{2}\right\rangle\right)\right\rangle\left(\left\langle\right.\right.$ dist $\left.\left._{2}, b_{1}\right\rangle,\left\langle s p_{50}, b_{2}\right\rangle\right)$ ) . 
Definition 5 A preference $\operatorname{Pref}(a)=(A, \geq)$ is consistent if and only if for all alternatives $x, y$ in $A$ s.t. their lists of propositional atoms differ exactly for one element, the following enumeration condition holds.

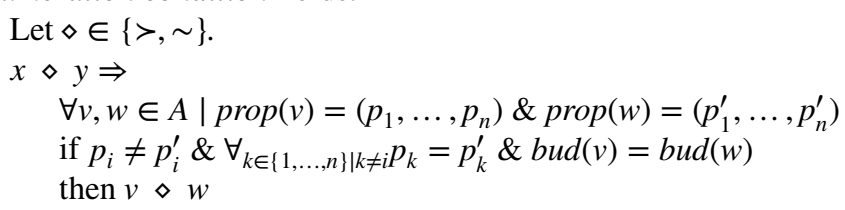

\subsection{Norms and agents' preferences}

As above mentioned, in this paper we assume that norms and agents' preferences are comparable. Consider $A L=\left(L_{1}, \ldots, L_{n}\right)$ and a norm set $\mathcal{N}=\left\langle N_{1}, \ldots, N_{n}\right\rangle$ as per Sect. 3.2. Given an alternative $\left(\left\langle p_{1}, b_{1}\right\rangle, \ldots,\left\langle p_{n}, b_{n}\right\rangle\right)$ in an agent's preference, we have that both the proposition $p_{i}$ of $i$-th pair $\left\langle p_{i}, b_{i}\right\rangle$ and the proposition $p$ of the $i$-th norm $N_{i}=(p, s)$ in $\mathcal{N}$ belong to $L_{i}$. Furthermore, since both the sanctions of the norms and the agents' budgets of agent's preferences are natural numbers, they also are commensurable. This makes it possible to analyze an agent's preference in the context of a norm to determine whether the preference motivates an agent to comply with a norm or to violate it.

Intuitively, in the context of a set of enforced norms, an agent that follows its preference aims at realizing a state of affairs that can be compliant with some of the enforced norms and violating other norms for which he is willing to pay the corresponding sanctions.

Given a set $\mathcal{N}$ of $n$ norms and a preference $(A, \geq)$, we say that an alternative $x \in A$ such that $x=\left(\left\langle p_{1}, b_{1}\right\rangle, \ldots,\left\langle p_{i}, b_{i}\right\rangle, \ldots,\left\langle p_{n}, b_{n}\right\rangle\right)$ is a violating alternative w.r.t. the $i$-th norm $N_{i}=(p, s)$ in $\mathcal{N}$, and we write $\operatorname{viol}\left(x, N_{i}\right)$, if and only if $p_{i}$ (e.g., $\left.s p_{100}\right)$ excludes $^{3} p$ (e.g., $s p_{50}$ ); otherwise $x$ is said to be a complying alternative w.r.t. norm $N_{i}$. An alternative that is compliant w.r.t. all norms in $\mathcal{N}$ is said fully compliant. Note that any rational preference, due to its completeness property as per Proposition 2, always contains at least one fully compliant alternative. This means that agents always have a choice to aim at a state of affairs that does not violate any norm.

Definition 6 (Most Preferred Alternatives to Act Upon) Given a preference $(A, \geq)$ and a set $\mathcal{N}$ of $n$ norms, a subset $A^{\prime} \subseteq A$ of alternatives is called the set of most preferred alternatives to act upon in the context of $\mathcal{N}$ if and only if for all alternatives $x \in A \backslash A^{\prime}$ it holds that for all alternatives $y \in A^{\prime}$ either $y>x$ or $x \geq y$ and there exists a norm $N_{j}=(p, s)$ in $\mathcal{N}$ s.t. $\operatorname{viol}\left(x, N_{j}\right) \& b_{j}<s$ (with $b_{j}$ budget of the $j$-th pair in $x$ ).

The set of most preferred alternatives to act upon in the context of $\mathcal{N}$ is the set of alternatives $A^{\prime} \subseteq A$ such that every other alternative $x \in A \backslash A^{\prime}$ is either strictly less preferred (i.e., $y>x \forall y \in A^{\prime}$ ), or is an alternative that violates at least a norm $N_{j}$ but the budget is not enough to pay the sanction (i.e., $\operatorname{viol}\left(x, N_{j}\right) \& b_{j}<s$ ). This means that the alternatives in $A^{\prime}$ are either fully compliant or they violate some norms and the budget is enough to pay

\footnotetext{
${ }^{3}$ In this paper we assume that information about exclusion between propositional atoms (e.g., in the sense of material implication) is given as background knowledge. A formal definition of violation of a norm depends on the specific language used to specify the norms and is out of the scope of the paper.
} 
the sanction, and there is no other alternative that satisfies such conditions that is strictly preferred to them.

A rational agent always acts upon one of its most preferred alternatives. We say that an agent $a$ has a reason to violate a norm $N$ whenever the agent's preference $\operatorname{Pref}(a)$ is so that, among the set of most preferred alternatives, there is at least one alternative $x$ such that $\operatorname{viol}(x, N)$. When different alternatives are equally preferred by an agent, the agent can freely choose to aim at any of them. This means that an agent that has a reason to violate a norm will not necessary aim to violate it: if another alternative is equally preferred to the violating state of affairs, the agent may decide to aim at to obeying state of affairs, despite it has a reason to violate the norm. Consider for example an agent type characterized by the preference in Eq. (1) and a norm $N=\left(s p_{50}, 0\right)$ that prohibits agents to drive faster than $50 \mathrm{~km} / \mathrm{h}$. Given $N$, the agents' most preferred alternatives to act upon are $\left(\left\langle s p_{100}, 0\right\rangle,\left\langle\right.\right.$ dist $\left.\left._{1}, 0\right\rangle\right),\left(\left\langle s p_{100}, 0\right\rangle,\left\langle\right.\right.$ dist $\left.\left._{2}, 0\right\rangle\right),\left(\left\langle s p_{50}, 0\right\rangle,\left\langle\right.\right.$ dist $\left.\left._{1}, 0\right\rangle\right)$ and $\left(\left\langle s p_{50}, 0\right\rangle,\left\langle\right.\right.$ dist $\left.\left._{2}, 0\right\rangle\right)$. Some of these alternatives violate the norm $N$ (e.g., $\left.\left(\left\langle s p_{100}, 0\right\rangle,\left\langle d i s t_{1}, 0\right\rangle\right)\right)$, therefore the agent has a reason to violate $N$. However, some of the other most preferred alternatives are compliant with the norm (e.g., $\left(\left\langle s p_{50}, 0\right\rangle,\left\langle\right.\right.$ dist $\left.\left.\left._{2}, 0\right\rangle\right)\right)$. Since all most preferred alternatives are equally preferred, the agent may rationally decide to aim at any of them.

We introduce the notion of maximum budget for norm violation as the maximal payment that an agent is willing to pay for violating a given norm according to its preference. Let $N_{i}=(p, s)$ be the $i$-th norm in $\mathcal{N}$, and let $\operatorname{Pref}(a)=(A, \geq)$ be the preference of agent $a$. Let $x \in A$ be the agent's most preferred fully compliant alternative, and $A^{\prime}=\{y \in A \mid y \geq x\}$ be the set of alternatives in $A$ that are (equally) preferred to $x$. The maximum budget that $a$ is willing to pay for the violation of $N_{i}$, denoted as $\max B\left(a, N_{i}\right)$, is the highest budget $b$ that occurs in the $i$-th pair of the alternatives in $A^{\prime}$. Note that if the maximum budget for violating a norm is lower than the sanction of norm $N_{i}$, then the most preferred alternatives to act upon are necessarily alternatives compliant w.r.t. $N_{i}$. For instance if $N=\left(s p_{50}, 3\right)$ and an agent $a$ has $\max B(a, N)=2$, then all alternatives $x$ in the set of most preferred alternatives are compliant to $N$, i.e., $\operatorname{viol}(x, N)$ does not hold, and it does not exists a pair $\langle p, b\rangle \in x$ with $b \geqslant 3$, since $b \leqslant \max B(a, N)<3$.

Finally, it is worth noting that in case of preference composed by more than one basic preference as per Definition 4, it is always the case that if the first basic preference is strictly preferred to the remaining ones then the set of most preferred alternatives to act upon in the context of $\mathcal{N}$ never contains any alternatives from any basic preference apart from the first one. This is because the first basic preference necessarily contains an alternative that is fully compliant (due to completeness of every basic preference $\left(A_{i}, \succeq_{i}\right)$ w.r.t. $A L$ and $B L_{i}$ for $1 \leqslant i \leqslant k$ and $k$ number of basic preferences composing the preference), and such alternative is strictly preferred to any other alternative that belongs to the remaining basic preferences.

\section{Norm-based supervision}

In this section we present the key concepts of a norm-based supervision of a multi-agent system. We build on the runtime norm-based supervision mechanism for multiagent systems as proposed in [23] and sketched in Fig. 2. Such mechanism corresponds to a control loop that continuously monitors the behavior of a multiagent system, evaluates the enforcement of the norms w.r.t. the system-level objectives, and, when needed, intervenes by revising the norms. 


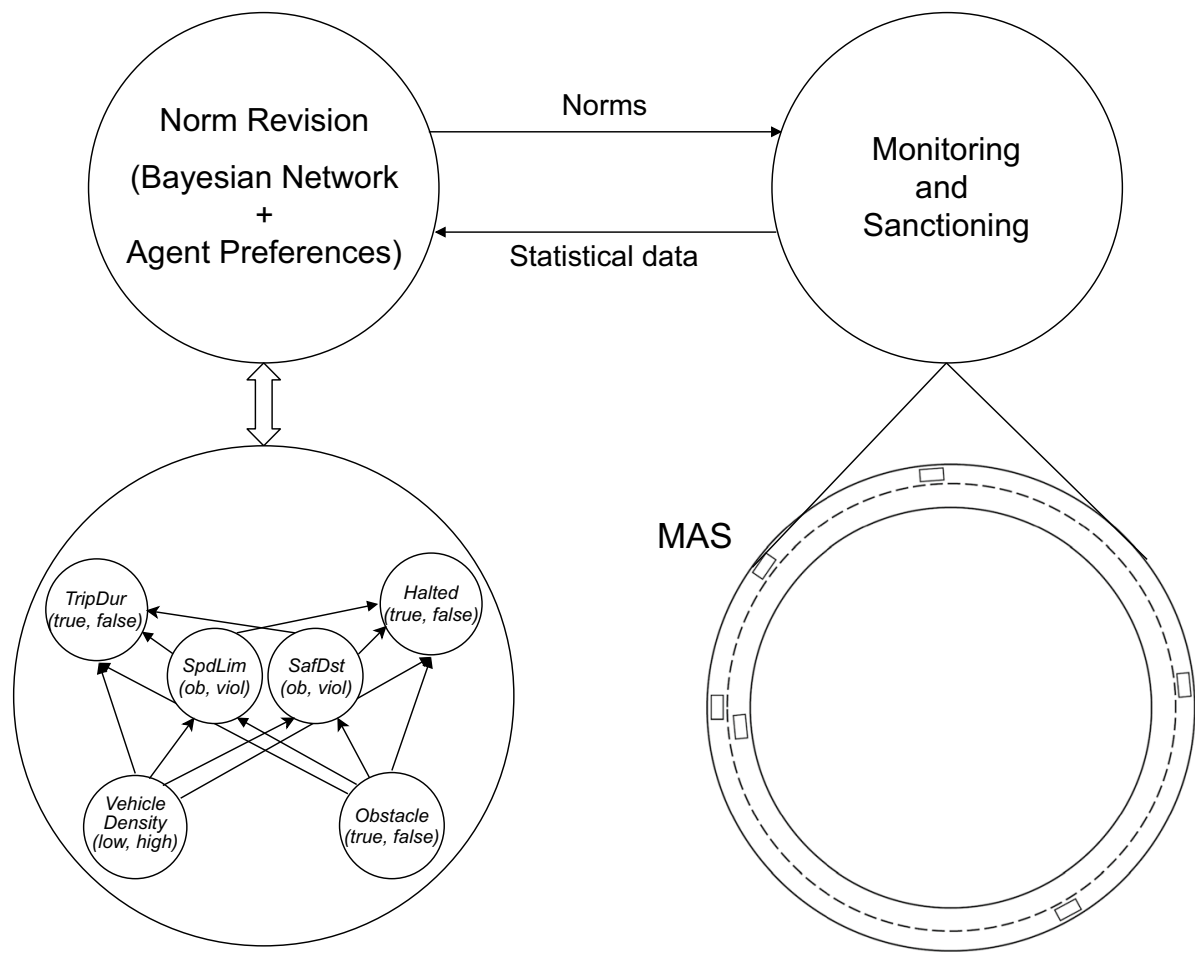

Fig. 2 Illustration of the MAS supervision mechanism

Consider an ordered set $\mathcal{N}=\left\langle N_{1}, \ldots, N_{n}\right\rangle$ of norms and a set $\mathcal{C}$ of all possible operating contexts of the multiagent system (e.g, a context $c \in \mathcal{C}$ in the ring road scenario could be "low vehicle density and no obstacle"). We call system configuration an assignment of a sanction $s \in \mathbb{N}$ to each norms in $\mathcal{N}$ in each of the MAS operating contexts.

For example, given two possible operating contexts $c_{1}$ and $c_{2}$, and given a norm set $\mathcal{N}=\left\{N_{1}, N_{2}\right\}$, a possible system configuration is $\left\{\left(c_{1},\left(N_{1}, 1\right),\left(N_{2}, 0\right)\right)\right.$, $\left.\left(c_{2},\left(N_{1}, 0\right),\left(N_{2}, 1\right)\right)\right\}$, meaning that in context $c_{1}$ norms $N_{1}$ and $N_{2}$ are enforced respectively with sanctions 1 and 0 , while in context $c_{2}$ they are enforced respectively with sanctions 0 and 1 .

The control loop of the supervision mechanism sketched in Fig. 2 starts with an initial system configuration. A Monitoring and Sanctioning component collects, at runtime, perfect information about the obedience or violation of the norms in the contexts in which they are evaluated and sanctions agents that violate the norms. Such component also provides a Boolean evaluation of the system-level objectives (e.g., whether the number of halted cars is below a certain threshold or not, in the ring road scenario).

The collected information is used to automatically train a Bayesian Network called Norm Bayesian Network (described in Sect. 4.1) that is used to learn and reason at runtime about the correlation between norm obedience or violation and the achievement of the system-level objectives. For example, the Norm Bayesian Network helps answering questions like how well, and in which contexts, does the norm $\left(s p_{50}, 100\right)$ help achieve the objective of avoiding halted cars? 
A Norm Revision component makes use of the learned knowledge, encoded in the Bayesian Network, to determine whether some norms should be revised and how. Revising a norm $N=(p, s)$ means modifying either the proposition $p$ or the sanction $s$, or both. In this paper we focus on the revision of the sanctions of the norms. The norm revision process generates as output a (possibly) new system configuration, replacing the current one.

In previous work [23], we proposed an implementation of the control loop above described as a variation of the hill climbing optimization technique. In this paper we follow the same approach. We consider the system configurations as possible solutions to explore in order to find an optimal one. The quality of a solution is determined, by means of the observed data from MAS execution, as the probability of achieving the system-level objectives. Instead of terminating the exploration of the space when a local optimum is found, as in traditional hill climbing, we use as stopping criterion a constraint defined by the system designer that determines whether or not the current solution is acceptable. In particular we use, as stopping criterion, a minimum desired value of the probability of achieving the system-level objectives. We call such value $t_{\text {oa }}$. We use the Norm Revision component to determine the next solution to try, when the current one is not acceptable.

In [23] we proposed heuristic algorithms for suggesting norm revisions that alter the regimented norms. In this paper, differently from the earlier work, we make use of some additional information concerning the preferences of the agents in order to determine how to revise the norms, and we focus on the revision of sanctions. In [24], we used the same framework of [23] to revise the way one norm is enforced by modifying its sanction. In this paper, we significantly extend our previous work by devising several new strategies for the revision of the sanctions of multiple norms enforced at the same time.

In the rest of the section we first provide some background concerning the Norm Bayesian Network, then we analyze some properties of the relationships between norms, agents' preferences and system-level objectives.

\subsection{Norm Bayesian network}

Consider some monitorable environmental properties such as the density of vehicles or the presence of an obstacle on the ring road. Each of these properties is called contextual variable, and is associated to a domain of values. For example, Vehicles density can be either low or high, while Obstacle can be true or false. Given a set of contextual variables, a context assigns a value to each contextual variable. For instance, given Vehicles density and Obstacle, four possible contexts exist: high-true, high-false, low-true, low-false.

A Norm Bayesian Network $\mathcal{N B N}=(\mathcal{X}, \mathcal{A}, \mathcal{P})[23]$ is a Bayesian Network where:

- $\mathcal{X}=\mathbf{N} \cup \mathbf{O} \cup \mathbf{C}$ are nodes that represent random variables in probability theory. $\mathbf{N}, \mathbf{O}$ and $\mathbf{C}$ are disjoint sets. $\mathbf{N}$ consists of norm nodes; each node $N \in \mathbf{N}$ corresponds to a norm and has a discrete domain of 3 possible values: obeyed, violated and disabled. $\mathbf{O}$ consists of objective nodes; each node $O \in \mathbf{O}$ corresponds to a Boolean objective and has a discrete domain of 2 values: true and false. Finally, $\mathbf{C}$ consists of context nodes; each node $C \in \mathbf{C}$ corresponds to a contextual variable and can have a discrete or continuous domain of values.

- $\mathcal{A} \subseteq(\mathbf{C} \times \mathbf{N}) \cup(\mathbf{C} \times \mathbf{O}) \cup(\mathbf{N} \times \mathbf{O})$ is the set of arrows that connect pairs of nodes. If there is an arrow from node $X$ to node $Y, X$ is called parent of $Y$.

- $\mathcal{P}$ is a set of conditional probability distributions. These are encoded into conditional probability tables (CPTs), each one associated with a node in $\mathcal{X}$ and quantifying the 
Fig. 3 A Norm Bayesian Network for the ring road

\begin{tabular}{llllll}
\hline VehicleDensity & Obstacle & SpdLim & SafDst & TripDur & Halted \\
\hline Low & true & viol & ob & true & false \\
Low & false & ob & viol & false & false \\
High & true & viol & ob & true & false \\
High & false & ob & ob & true & true \\
$\ldots$ & & & & & \\
\hline
\end{tabular}

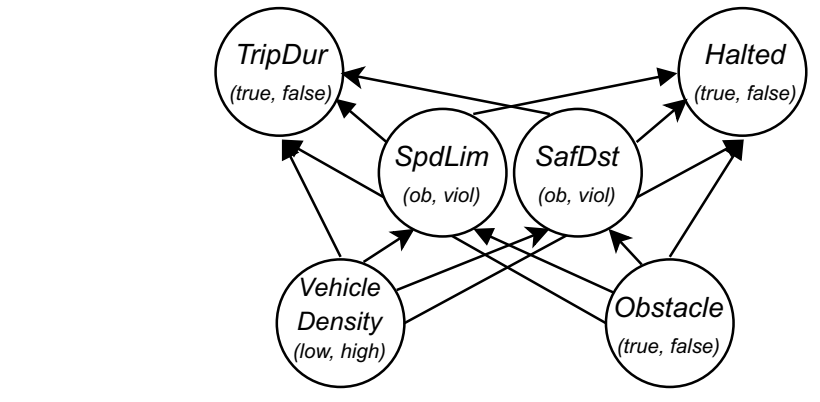

Table 1 Example of part of a dataset used to train the Norm Bayesian Network of Fig. 3 and obtained from monitoring the execution of the MAS

effect of the parents on the node. The conditional probability values in the CPT of a node are the parameters of the network. These parameters are automatically learned from observed data from MAS execution through classic Bayesian learning.

Notation In the rest of the paper, we use the following notation for Bayesian Networks. Italic uppercase $(X, Y, \ldots)$ for random variables; bold uppercase $(\mathbf{X}, \mathbf{Y}, \ldots)$ for sets of random variables; italic lowercase $\left(v_{1}, v_{2}, \ldots\right)$ for values in the domain of a random variable; $N_{v}$ abbreviates $(N=v)$, i.e., an assignment of value $v$ to a norm variable $N ; \mathbf{O}_{v}$ denotes an assignment of value $v$ to all nodes in $\mathbf{O} ; P$ denotes a single probability. An evidence $\mathbf{e}$ is an observed assignment of values for some or all of the random variables in the network. An evidence $\mathbf{c}$ for all the context nodes $\mathbf{C}$ is an observation for a certain context; for example, Vehicles density has value low and Obstacle has value false. For simplicity, we use the term context also to refer to the associated evidence in the Bayesian Network.

Figure 3 reports an example of a Norm Bayesian Network for the running example of the ring road.

Since we focus on revising the sanctions that enforce norms, norms are never disabled, therefore in the following we ignore the disabled value of the nodes in the Bayesian Network. Despite we do not explicitly disable a norm, we consider enforcing a norm with a sanction of 0 as equivalent to disabling the norm, assuming that an agent that violates a norm with sanction of 0 does not incur in any other kind of sanctions (e.g., consequences in the relation between the individual and the other agents due to shared (moral) values [7]).

Finally, the construction and training of the Norm Bayesian Network is a fully automated process. In particular, the structure of the network can be trivially obtained from the the definition of $\mathcal{X}$ and $\mathcal{A}$. The conditional probability distributions $\mathcal{P}$ (i.e., the parameters of the network), instead, are automatically learned through classical Bayesian learning using data collected from MAS execution. Without going into the details of the Monitoring and 
Sanctioning component, which are out of the scope of this paper, Table 1 reports a sample dataset that can be obtained from monitoring norms and objectives for the running example of the ring road. The values that each of the variables assumes belongs to its domain as above specified (e.g., obeyed, violated, for norm nodes, true or false for objective nodes). Such dataset can be used to automatically train the Norm Bayesian Network of Fig. 3 and learn the set of conditional probability distributions $\mathcal{P}$. As in this work we assume that the population of agents do not change over time and that the behavior of agents is consistent over time, the CPTs of the Norm Bayesian Network stabilize after receiving a sufficient number of evidences.

\subsection{Norms, agents' preferences and system-level objectives in MAS}

Consider a set of agent types $\mathcal{T}=\left\{t_{1}, \ldots, t_{k}\right\}$, each type corresponding to a preference as per Sect. 3. In order to focus on the revision of the norms' sanctions, we assume that we possess a correct estimation of the preferences of agents concerning the aspects of the system we aim to regulate. Additionally, we assume that the agents' preferences do not change in different contexts. As we will see in the following, an accurate estimation of agents' preferences is helpful for improving the effectiveness of our heuristics. Our technique, however can be extended to support partial or inaccurate estimations of the agents' preferences. In Sect. 7.1, we sketch some directions for future work to support these aspects.

Take a set of agents $A g=\left\{a_{1}, \ldots, a_{n}\right\}$, each with a specific type from $\mathcal{T}$. We use $\operatorname{Pref}(a) \in \mathcal{T}$ to indicate that agent $a \in A g$ behaves according to a type from $\mathcal{T}$. For simplicity we assume that the behaviors exhibited in the multiagent system are uniformly distributed over all the agents: at every time instant every agent either violates or obeys each of the enforced norms.

Given these assumptions and a set of norms $\mathcal{N}$, we say that a norm $N$ in $\mathcal{N}$ is well defined in the context of $\mathcal{N}$ (simply well defined, for brevity) if the probability that $N$ is violated, denoted as $P\left(N_{\text {viol }}\right)$, is never higher than the percentage of agents in the MAS with a reason to violate $N$ in the context of $\mathcal{N} .{ }^{4}$ In other words, the upper bound of the probability $P\left(N_{\text {viol }}\right)$ in the context of $\mathcal{N}\left(\right.$ denoted as $\left.U B\left(N_{\text {viol }}, \mathcal{N}\right)\right)$ is the percentage of the agents with a reason to violate $N$ in the context of $\mathcal{N}$.

Let $N$ be a norm in $\mathcal{N}$, and let $\delta=\left(d_{1}, \ldots, d_{k}\right)$ be a distribution over the agent types $\mathcal{T}=\left\{t_{1}, \ldots, t_{k}\right\}$, where $d_{i} \in[0,1]$ is the percentage of population of agents of type $t_{i}$, with $\sum_{i=1}^{k} d_{i}=1$. The percentage of agents with a reason to violate $N$ (as per Sect. 3.4) in the context of $\mathcal{N}$ is $\sum_{i=1}^{k}\left(d_{i} \cdot\right.$ hasReason $\left.(i, N, \mathcal{N})\right)$, with hasReason $(i, N, \mathcal{N})=1$ if agent type $t_{i}$ has a reason to violate $N$ in the context of $\mathcal{N}, O$ otherwise.

Consider, as an example, a norm set $\mathcal{N}=\left\langle N_{1}, N_{2}\right\rangle$, with $N_{1}=\left(s p_{50}, s_{1}\right)$ and $N_{2}=\left(\right.$ dist $\left._{2}, s_{2}\right)$ and $\mathcal{B}=\{0,1\}$. Consider the two types of agents $t_{1}$ and $t_{2}$ as per Eq. (1) and Eq. (2), respectively. Assuming a uniform distribution of agents between the two types,

\footnotetext{
${ }^{4}$ Consider a norm $N=$ every vehicle on the ring road shall always exceed $70 \mathrm{~km} / \mathrm{h}$, a type of norm employed in our society, for instance, to prevent vehicles to have negative impact on road throughput and safety. Our framework supports such type of norm if it is well-defined. Suppose that in our running example no agent has reason to violate $N$. If $N$ was well-defined we would expect $P\left(N_{\text {viol }}\right)=0$. However, in our running example, such norm is not well-defined, for in case of high density, for example, the agents may be forced to slow down below the minimum speed, therefore violating the norm and exhibiting $P\left(N_{\text {viol }}\right)>0$. A well-defined norm guarantees agents that have no reason to violate the norm (i.e., their preferred alternatives are compliant with the norm) to be able to obey such norm.
} 
Fig. 4 Upper bound of the probability of violating norms $N_{1}=\left(s p_{50}, s_{1}\right)$ (in red) and $N_{2}=\left(\right.$ dist $\left._{2}, s_{2}\right)$ (in black) with the two types of agents $t_{1}$ and $t_{2}$ as per Eqs. (1) and (2), respectively, uniformly distributed

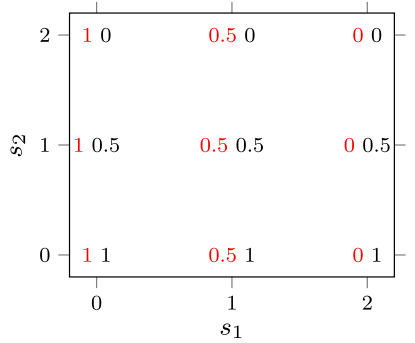

Fig. 4 reports the upper bound of the probability of violating $N_{1}$ and $N_{2}$ for this example with different sanctions (i.e., different values of $s_{1}$ and $s_{2}$ ).

The upper bound of $P\left(N_{\text {viol }}\right)$ describes a worst-case hypothetical situation where all agents behave according to their preferences, and if they have reason to violate a norm they are assumed to violate it, no contextual factor influences agent behavior, and interactions among agents do not prevent them to act according to their preferences. This would happen, for example, when a single car drives on an empty highway with perfect road and car conditions. Note, however, that the actual probability to violate a norm is affected by the agents' decisions, their interactions and by the MAS environment, and it is assumed to be unknown a priori. Even if all agents have a reason to violate a norm, due to their interaction or to environmental circumstances (e.g., large number of cars on the ring-road), none of them may end up violating it. Furthermore, as explained in Sect. 3.4, if an agent equally prefers two states of affairs, one violating a norm, and another obeying the norm, the agent, since autonomous, may decide to obey the norm even if it has a reason to violate it. We call, therefore, the monitored probability of violating (obeying) a norm exhibited norm violation (obedience). We do not assume any prior knowledge about such probability.

Note that, since we consider agent types with rational preferences as per Sect. 3.3, increasing the sanction $s$ of a norm $N=(p, s)$, without changing the sanctions of other norms, does not increase the percentage of agents with a reason to violate $N$. Therefore, given $k$ agent types and $\max B(\mathcal{T}, N)$ as the maximum budget among all agent types to violate a well-defined norm $N=(p, s)$, the percentage of agents with a reason to violate a well-defined norm $N^{\prime}=(p, \max B(\mathcal{T}, N)+1)$ in the context of $\mathcal{N}$ is 0 . This is to say that increasing the sanction of a norm above the maximum budget that any agent is willing to pay causes all agents to comply with the norm. Consequently, given two well-defined norms $N=\left(p, s_{1}\right)$ and $N^{\prime}=\left(p, s_{2}\right)$ such that $s_{2}>s_{1}$, and assuming no change in other norms of $\mathcal{N}$, the upper bound of the probability $P\left(N_{\text {viol }}^{\prime}\right)$ is never bigger than the upper bound of the probability $P\left(N_{\text {viol }}\right)$.

Furthermore, it is possible to prove that, if all agents in the MAS have a consistent preference (as per Definition 5), then given a set of norms $\mathcal{N}=\left\langle N_{1}, \ldots, N_{n}\right\rangle$, increasing the sanction of a norm $N_{j}$ in $\mathcal{N}$ without changing the sanctions of other norms, does not increase the upper bound of the probability $P\left(N_{\text {viol }}\right)$ for every $N$ in $\mathcal{N}$.

Proposition 3 Given an ordered set of norms $\mathcal{N}=\left\langle N_{1}, \ldots, N_{n}\right\rangle$, and a set of t agent types $\mathcal{T}$, each type corresponding to a consistent preference (as per Definition 5), increasing the sanction of a norm $N_{j}$ in $\mathcal{N}$ without changing the sanctions of other norms, does not increase the upper bound of the probability $P\left(N_{\text {viol }}\right)$, i.e., $U B\left(N_{\text {viol }}, \mathcal{N}\right)$, for all $N$ in $\mathcal{N}$.

Proof See “Appendix 1". 
The concept of well-defined norm as described above, concerns the relationship between a norm and the preferences of the agents. In a multiagent system, norms are enforced in order to achieve some system-level objectives. Although setting the sanction of all norms in $\mathcal{N}$ above $\max (\mathcal{B})$ makes all the agents fully compliant (i.e., $P\left(N_{\text {viol }}\right)=0$ and $P\left(N_{o b}\right)=1$ for all $N \in \mathbf{N}$ ), this does not necessarily guarantee the achievement of the system-level objectives, as norms can be ineffective, or even harmful, when obeyed by all agents [23]. Having an estimation of the agents' preferences on its own is therefore not sufficient for an effective supervision of a MAS.

We describe here two properties that, instead, relate a norm with the system-level objectives: the concept of synergy between a norm and the system-level objectives, and the concept of effectiveness of a norm set.

We say that there is a positive synergy between a norm and the system-level objectives if it is more likely to achieve the system-level objectives when the norm is obeyed than when it is violated. A positive synergy between a norm $N$ and a set of Boolean objectives $\mathbf{O}$ exists if $P\left(\mathbf{O}_{\text {true }} \mid N_{o b}\right)>P\left(\mathbf{O}_{\text {true }} \mid N_{\text {viol }}\right)$. We say that there is a negative synergy between $N$ and $\mathbf{O}$ if $P\left(\mathbf{O}_{\text {true }} \mid N_{o b}\right)<P\left(\mathbf{O}_{\text {true }} \mid N_{\text {viol }}\right)$. Finally, we say that there is no synergy between $N$ and $\mathbf{O}$ if $P\left(\mathbf{O}_{\text {true }} \mid N_{\text {ob }}\right)=P\left(\mathbf{O}_{\text {true }} \mid N_{\text {viol }}\right)$.

We say, instead, that a norm set $\mathcal{N}$ is effective if, when norms in $\mathcal{N}$ are enforced, $\mathcal{N}$ guarantees the desired achievement level $t_{o a}$ of the system-level objectives, i.e., when $P\left(\mathbf{O}_{\text {true }}\right) \geqslant t_{\text {oa }}$. Conversely, if, when enforcing a norm set $\mathcal{N}$, we have that $P\left(\mathbf{O}_{\text {true }}\right)<t_{\text {oa }}$, we say that $\mathcal{N}$ is ineffective.

Information such as the exhibited norm obedience, the synergy and the effectiveness described above, are hard to determine while designing a MAS. This is due to several factors, including the complexity of the system, the interaction between autonomous agents, the lack of complete knowledge of the agents' internals, and the uncertainty of the environment. However, they can be learned at runtime by monitoring the MAS execution. In this paper, we learn such properties by means of the Norm Bayesian Network and, in Sect. 5, we propose different strategies to combine these properties with the agents' preferences, in order to revise the sanctions of an ineffective norm set $\mathcal{N}$.

\section{Norm revision}

In this section we propose different heuristic strategies for the revision of the sanctions of a set of norms whose enforcement is currently ineffective (as per Sect. 4.2). Opportune sanctioning of agents is a well-known mechanism to achieve the system-level objectives in MASs [11, 12]. Our strategies leverage the knowledge learned at runtime about norm effectiveness and an estimation of the preferences of the agents in the system, and determine a new set of sanctions to use to enforce the norms.

Take the Norm Bayesian Network in Fig. 3. By analyzing the CPTs of the objectives nodes $\mathbf{O}=\{$ TripDur, Halted $\}$, we can determine whether a norm set $\mathcal{N}$ is effective or not in a context c. If $\mathcal{N}$ is not effective (i.e., $\left.P\left(\mathbf{O}_{\text {true }} \mid \mathbf{c}\right)<t_{o a}\right)$, a norm revision process is triggered. In such a case, in this paper we aim to revise the sanctions of the norms in $\mathcal{N}$. For example, if the two norms $\left(s p_{50}, 1\right)$ and $\left(\right.$ dist $\left._{1}, 1\right)$ are ineffective when on the ring road there is an obstacle and high vehicle density, we aim to identify another set of values for their sanctions. Given a norm set $\mathcal{N}$ consisting of $n$ norms, a set of agent types $\mathcal{T}$ and the maximum possible budget $\max (\mathcal{B})$ among all agent types in $\mathcal{T}$, the possible sets of sanctions that can be used to enforce norms in $\mathcal{N}$ is $\mathcal{S}=\times_{i=1}^{n}\{s \in \mathbb{N} \mid s \leqslant \max (\mathcal{B})+1\}$. When a norm is enforced with a sanction 
0 , agent's decisions are not affected by the norm, since every agent can always afford to violate (if preferred) a norm with sanction 0 . When a norm is enforced with a sanction $\max (\mathcal{B})+1$, instead, no agent can violate such norm, since no agent can afford to pay such sanction, for the maximum possible budget among all agent types is $\max (\mathcal{B})$.

The set $\mathcal{S}$ is the search space within which our heuristic strategies for norm revision search for new sanctions.

In Sect. 5.1, we describe six strategies for the suggestion of a revision of the sanctions of a norm set. Such strategies extend and adapt heuristics presented in previous work [23, 24] by supporting the revision of sanctions of multiple norms. Each strategy suggests how the behavior of agents w.r.t. the aspects of the system regulated by norms should change in order to improve the probability of achieving the system-level objectives. For example, given two norms, one strategy could suggest to reduce the violations of one norm and to increase the violations of the second norm. Based on the upper bound of the violation of norms obtained from agents' preferences (Sect. 4.2), we provide then in Sect. 5.2 an algorithm to explore the search space $\mathcal{S}$ in order to identify a new set of sanctions that satisfies (as much as possible) the suggestions provided by the revision strategies.

It is worth noting that we do not claim that modifying sanctions is always enough in order to achieve the system's objectives. As shown in previous work [23], sometimes the enforced norms (and not their enforcement) need to be revised. In this paper, however, we focus on mechanisms for the revision of the sanctions associated to the norms (i.e., the way norms are enforced). The combination of the mechanisms proposed here with the revision of the content of the norms is left for future work.

\subsection{Norm revision strategies}

We propose six strategies for the suggestion of norm revisions. Each strategy determines a list of $n$ suggestions (one per each norm in $\mathcal{N}$ ). We present three types of strategies: synergybased strategies, sensitivity-based strategies, and category-based strategies.

Each strategy is applied to a context mpc that, in our framework, corresponds to the most problematic context in which the objectives are not achieved. In particular, $\mathbf{m p c}=\operatorname{argmax}_{\mathbf{c} \in \operatorname{all}(\mathbf{c})} P\left(\mathbf{O}_{\text {false }} \mid \mathbf{c}\right)$, where $\operatorname{all}(\mathbf{c})$ is the set of all possible contexts (assignments of a value to each of the context nodes in $\mathcal{N B N}$ ). For simplicity, in the rest of the section, we call such context simply c.

\subsubsection{Synergy-based strategies}

Synergy-based strategies are based on the concept of norm-objectives synergy described in Sect. 4.2. The idea is that, if there is a positive synergy between a norm $N$ and the objectives $\mathbf{O}$ in $\mathbf{c}$, the objectives $\mathbf{O}$ are more likely to be achieved when $N$ is obeyed. In this case, by reducing the violations of $N$, we expect to increase $P\left(\mathbf{O}_{\text {true }} \mid \mathbf{c}\right)$. If there is a negative synergy between $N$ and $\mathbf{O}$ in $\mathbf{c}$, instead, we expect that increasing the violations of $N$, and $P\left(\mathbf{O}_{\text {true }} \mid \mathbf{c}\right)$ would increase. We present two strategies of this type (Naive synergy and Combined synergy), which differ in the way they determine the synergy between norms and objectives.

Naive synergy Consider, for each norm $N \in \mathbf{N}$, its synergy with the objectives $\mathbf{O}$ :

$$
\operatorname{argmax}_{v \in\{\text { ob,viol }\}} P\left(\mathbf{O}_{\text {true }} \mid N_{v} \wedge \mathbf{c}\right)
$$

For instance, for a norm node SpdLim in the Bayesian Network of Fig. 3, where $\mathbf{O}=\{$ TripDur, Halted $\}$, we have that 


$$
\begin{aligned}
& P\left(\mathbf{O}_{\text {true }} \mid N_{v} \wedge \mathbf{c}\right)=P\left(\text { TripDur }_{\text {true }}, \text { Halted }_{\text {true }} \mid\right. \\
& \left.\operatorname{SpdLim}_{v} \wedge \mathbf{c} \wedge \text { SafDst }_{\text {ob }}\right) \cdot P\left(\text { SafDst }_{o b} \mid \mathbf{c}\right)+P\left(\text { TripDur }_{\text {true }}, \text { Halted }_{\text {true }} \mid\right. \\
& \text { SpdLim } \left._{v} \wedge \mathbf{c} \wedge \text { SafDst }_{\text {viol }}\right) \cdot P\left(\text { SafDst }_{\text {viol }} \mid \mathbf{c}\right)
\end{aligned}
$$

To determine the argmax of Eq. (4) means therefore to determine if SpdLim ${ }_{o b}$ is better than SpdLim $_{\text {viol }}$ for the achievement of the objectives TripDur and Halted.

Naive synergy calculates such argmax for each norm node and suggests to decrease violations of norms such that $v=o b$ in Eq. (4), and to increase violations of norms where $v=$ viol in Eq. (4). For instance, given $\mathcal{N}=\langle N 1, N 2\rangle$, if $v=o b$ for $N 1$ and $v=$ viol for $N 2$, then naive synergy suggests to decrease violations of norm $N 1$ and to increase violations of norm $N 2$.

Combined synergy Determine which combination of values obeyed and violated for each norm is the best for the achievement of the objectives $\mathbf{O}$.

Let $\mathbf{o v}$ be the set of all possible assignments of values in the set $\{o b, v i o l\}$ to all norm nodes in $\mathbf{N}$ (e.g., given $\mathbf{N}=\{N 1, N 2\}$, then $\mathbf{o v}=\left\{\left\{N 1_{o b}, N 2_{o b}\right\},\left\{N 1_{o b}, N 2_{\text {viol }}\right\}\right.$, $\left.\left.\left\{N 1_{\text {viol }}, N 2_{o b}\right\},\left\{N 1_{\text {viol }}, N 2_{\text {viol }}\right\}\right\}\right)$. Determine:

$$
\mathbf{n}_{d}=\operatorname{argmax}_{\mathbf{n} \in \mathbf{o v}} P\left(\mathbf{O}_{\text {true }} \mid \mathbf{n} \wedge \mathbf{c}\right)
$$

This strategy suggests to decrease violations of norms with value $o b$ in $\mathbf{n}_{d}$ and to increase violations of norms with value viol in $\mathbf{n}_{d}$. For instance if $\mathbf{n}_{d}=\left\{N 1_{o b}, N 2_{\text {viol }}\right\}$, then combined synergy suggests to increase violations of norm $N 1$ and to decrease violations of norm $N 2$.

It is worth noting that Combined synergy purely determines the best combination of values for the norms, according to the observed data from MAS execution, without considering the prior probability of observing those values (in practice, Combined synergy only compares, one by one, the rows of the CPT of the objective nodes). Naive synergy, instead, when comparing different combinations of values for the norms, takes also into account the probability to observe those values (Naive synergy compares sums of different rows of the CPT of the objectives nodes, multiplied by the prior probability of observing the corresponding values for the norm nodes). Adopting the Naive synergy strategy may have the advantage of providing more precise suggestion w.r.t. the data acquired so far during the system execution. Considering only the CPT of the objective nodes, as per Combined synergy, may help instead determining the actual best combination of values of obedience of the norms for the system-level objectives, without being biased by the current probabilities of violating the norms, which will be modified after the sanctions revision.

\subsubsection{Sensitivity-based strategies}

Sensitivity-based strategies are based on the sensitivity analysis technique from probabilistic reasoning [14]. Such strategies do not only determine the direction of the revision-i.e., increasing or decreasing the probability of violating a norm, as in the case of synergy-based strategies-, but also estimate the required change in such probability in order to make the entire norm set effective in context c. In particular, given a norm node $N$, the probability $P\left(N_{\text {viol }} \mid \mathbf{c}\right)$ is a parameter $\theta_{N_{\text {violl }}}$ of the Norm Bayesian Network. Sensitivity-based strategies try to identify possible changes to the parameter $\theta_{N_{\text {viols }}}$ that can ensure the satisfaction of the constraint $P\left(\mathbf{O}_{\text {true }} \mid \mathbf{c}\right) \geqslant t_{\text {oa }}$. We call required revision strength $(R R S)$ for a norm set $\mathcal{N}=\langle N 1, \ldots N n\rangle$, the set of desired changes $\left\{\Delta \theta_{N 1_{\text {viol } \mid \mathrm{c}}}, \ldots, \Delta \theta_{N n_{\text {viol }] \mathrm{c}}}\right\}$ in the parameters $\theta_{N_{\text {viol }}}$ of each $N$ in $\mathcal{N}$ that ensure the satisfaction 
of the constraint $P\left(\mathbf{O}_{\text {true }} \mid \mathbf{c}\right) \geqslant t_{\text {oa }}$. We present two strategies of this type (Naive sensitivity analysis and $n-C P T$ sensitivity analysis), which differ in the way they determine such set of desired changes for each norm in $\mathcal{N}$.

Naive sensitivity analysis Determine, for each norm $N$, the required revision strength (RRS) $\Delta \theta_{N_{\text {viol }}}$ by solving Eq. (6).

$$
P\left(\mathbf{O}_{\text {true }} \mid \mathbf{c}\right)+\frac{\delta P\left(\mathbf{O}_{\text {true }} \mid \mathbf{c}\right)}{\delta \theta_{N_{\text {viol } \mid \mathbf{c}}}} \cdot \Delta \theta_{N_{\text {viol } \mid \mathbf{c}}} \geqslant t_{o a}
$$

Consider the topology of a Norm Bayesian Network. Following Chan et al. [14], the derivative $\frac{\delta P\left(\mathbf{O}_{\text {true }} \mid \mathbf{c}\right)}{\delta \theta_{N_{\text {viol }} \mathbf{c}}}$ for a norm node $N$ in $\mathcal{N}$ can be computed as follows.

$$
\frac{\delta P\left(\mathbf{O}_{\text {true }} \mid \mathbf{c}\right)}{\delta \theta_{N_{\text {viol| }}}}=\frac{P\left(\mathbf{O}_{\text {true }}, N_{\text {viol }} \mid \mathbf{c}\right)}{P\left(N_{\text {viol }} \mid \mathbf{c}\right)}-P\left(\mathbf{O}_{\text {true }} \mid N_{\text {ob }}, \mathbf{c}\right)
$$

For instance, for a norm node SpdLim in the Bayesian Network of Fig. 3, where $\mathbf{O}=\{$ TripDur, Halted $\}$, the left member of the difference in Eq. (7) is

$$
\begin{aligned}
& \frac{P\left(\mathbf{O}_{\text {true }}, N_{\text {viol }} \mid \mathbf{c}\right)}{P\left(N_{\text {viol }} \mid \mathbf{c}\right)}=\frac{P\left(\text { TripDur }_{\text {true }}, \text { Halted }_{\text {true }}, \text { SpdLim }_{\text {viol }} \mid \mathbf{c}\right)}{P\left(\text { SpdLim }_{\text {viol }} \mid \mathbf{c}\right)} \\
& =\frac{P\left(\operatorname{TripDur}_{\text {true }}, \text { Halted }_{\text {true }} \mid \operatorname{SpdLim}_{\text {viol }}, \mathbf{c}\right) \cdot P\left(\operatorname{SpdLim}_{\text {viol }} \mid \mathbf{c}\right)}{P(\operatorname{SpdLim} \text { viol } \mid \mathbf{c})}=P\left(\operatorname{TripDur}_{\text {true }} \mid \operatorname{SpdLim}_{\text {viol }}, \mathbf{c}\right) \cdot P\left(\text { Halted }_{\text {true }} \mid \operatorname{SpdLim}_{\text {viol }}, \mathbf{c}\right) \\
& =P\left(\text { TripDur }_{\text {true }} \mid \text { SpdLim }_{\text {viol }}, \text { SafDst }_{\text {viol }}, \mathbf{c}\right) \cdot P\left(\text { Halted }_{\text {true }} \mid \text { SpdLim }_{\text {viol }}, \text { SafDst }_{\text {viol }}, \mathbf{c}\right) \cdot P\left(\text { SafDst }_{\text {viol }} \mid \mathbf{c}\right) \\
& +P\left(\text { TripDur }_{\text {true }} \mid \text { SpdLim }_{\text {viol }}, \text { SafDst }_{o b}, \mathbf{c}\right) \cdot P\left(\text { Halted }_{\text {true }} \mid \text { SpdLim }_{\text {viol }}, \text { SafDst }_{o b}, \mathbf{c}\right) \cdot P\left(\text { SafDst }_{o b} \mid \mathbf{c}\right)
\end{aligned}
$$

while the right member of the difference in Eq. (7) is

$$
\begin{aligned}
P\left(\mathbf{O}_{\text {true }} \mid N_{\text {ob }}, \mathbf{c}\right)= & P\left(\text { TripDur }_{\text {true }}, \text { Halted }_{\text {true }} \mid \operatorname{SpdLim}_{o b}, \mathbf{c}\right) \\
= & P\left(\text { TripDur }_{\text {true }} \mid \operatorname{SpdLim~}_{o b}, \text { SafDst }_{\text {viol }}, \mathbf{c}\right) \cdot P\left(\text { Halted }_{\text {true }} \mid \operatorname{SpdLim~}_{o b}, \text { SafDst }_{\text {viol }}, \mathbf{c}\right) \cdot P\left(\text { SafDst }_{\text {viol }} \mid \mathbf{c}\right) \\
& +P\left(\text { TripDur }_{\text {true }} \mid \operatorname{SpdLim~}_{o b}, \text { SafDst }_{o b}, \mathbf{c}\right) \cdot P\left(\text { Halted }_{\text {true }} \mid \operatorname{SpdLim~}_{o b}, \text { SafDst }_{o b}, \mathbf{c}\right) \cdot P\left(\text { SafDst }_{o b} \mid \mathbf{c}\right)
\end{aligned}
$$

Therefore the derivative of Eq. (7) for a norm node SpdLim in the Bayesian Network of Fig. 3 can be computed as:

$$
\begin{aligned}
& P\left(\text { TripDur }_{\text {true }} \mid \text { SpdLim }_{\text {viol }}, \text { SafDst }_{\text {viol }}, \mathbf{c}\right) \cdot P\left(\text { Halted }_{\text {true }} \mid \text { SpdLim }_{\text {viol }}, \text { SafDst }_{\text {viol }}, \mathbf{c}\right) \cdot P\left(\text { SafDst }_{\text {viol }} \mid \mathbf{c}\right) \\
& +P\left(\text { TripDur }_{\text {true }} \mid \text { SpdLim }_{\text {viol }}, \text { SafDst }_{o b}, \mathbf{c}\right) \cdot P\left(\text { Halted }_{\text {true }} \mid \text { SpdLim }_{\text {viol }}, \text { SafDst }_{o b}, \mathbf{c}\right) \cdot P\left(\text { SafDst }_{o b} \mid \mathbf{c}\right) \\
& -P\left(\text { TripDur }_{\text {true }} \mid \operatorname{SpdLim}_{o b}, \text { SafDst }_{\text {viol }}, \mathbf{c}\right) \cdot P\left(\text { Halted }_{\text {true }} \mid \operatorname{SpdLim}_{o b}, \text { SafDst }_{\text {viol }}, \mathbf{c}\right) \cdot P\left(\text { SafDst }_{\text {viol }} \mid \mathbf{c}\right) \\
& -P\left(\text { TripDur }_{\text {true }} \mid \operatorname{SpdLim}_{o b}, \text { SafDst }_{o b}, \mathbf{c}\right) \cdot P\left(\text { Halted }_{\text {true }} \mid \operatorname{SpdLim}_{o b}, \text { SafDst }_{o b}, \mathbf{c}\right) \cdot P\left(\text { SafDst }_{o b} \mid \mathbf{c}\right)
\end{aligned}
$$

The $R R S$ for a norm $N$ determines the change in $P\left(N_{\text {viol }} \mid \mathbf{c}\right)$ that is estimated, based on observed data from MAS execution, to be required in order to make the norm set $\mathcal{N}$ effective.

Naive sensitivity analysis suggests to change (increase or decrease) the violations of norms of the amount determined by the corresponding RRSs. The sign of the required revision strength determines whether it is required to reduce (negative $R R S$ ) or to increase (positive $R R S$ ) violations of a norm, i.e., it determines the direction of the required revision. The value of the $R R S$ determines the intensity of the required change. For instance if $\Delta \theta_{N 1_{\text {viole }}}=+0.2$ and $\Delta \theta_{N 2_{\text {viole }}}=-0.5$, then the suggestion is to increase $P\left(N 1_{\text {viol }}\right)$ of 0.2 and to decrease $P\left(N 2_{\text {viol }}\right)$ of 0.5 .

This strategy computes the $R R S$ for a norm, without considering that a change could be applied, at the same time, also to other norms. In other words, the $R R S$ for a norm $N$ 
is computed as if no change in the probability of violating any other norm could happen (from this the term naive). However, when determining the RRS for a norm, Naive sensitivity analysis considers all possible values of the other norms. Therefore, this strategy may result robust to unexpected changes in the probability of violating other norms when changing the sanctions.

$n-C P T$ sensitivity analysis Determine the required revision strength for all norms together, by solving, following Chan et al. [14], Eq. (8) for the $n$ parameters $\Delta \theta_{N 1_{\text {viol }}}, \ldots, \Delta \theta_{N n_{\text {viol }}}$. Let $c o(\mathbf{N}, i)$ be the set of all possible combinations of $i$ norm nodes from the set $\mathbf{N}$, and, given a set $\mathbf{M}=\{N 1, \ldots, N m\} \subseteq \mathbf{N}$ of norm nodes, let $\frac{\delta^{i}}{\delta \theta \mathbf{M}_{\text {viollc }}}$ be the Leibniz's notation for the $i$-th partial derivative $\frac{\delta^{i}}{\delta \theta N 1_{\text {viollc }} \ldots \delta \theta N m_{\text {viol }}}$ for $N_{j} \in \mathbf{M}$.

$$
P\left(\mathbf{O}_{\text {true }} \mid \mathbf{c}\right)+\sum_{i=1}^{n}\left[\sum_{\mathbf{M} \in c o(\mathbf{N}, i)}\left(\frac{\delta^{i} P\left(\mathbf{O}_{\text {true }} \mid \mathbf{c}\right)}{\delta \theta \mathbf{M}_{\text {viol } \mid \mathbf{c}}} \cdot \prod_{N \in \mathbf{M}} \Delta \theta_{N_{\text {viol } \mid \mathbf{c}}}\right)\right] \geqslant t_{o a}
$$

Solving Eq. (8) means to determine a list of $n$ values $\Delta \theta_{N_{\text {viol }}}$, one for each norm node $N \in \mathbf{N}$. To do so, first of all it is required to compute: the $n$ first partial derivatives $\frac{\delta P\left(\mathbf{O}_{\text {true }} \mid \mathbf{c}\right)}{\delta \theta_{N_{\text {viol }} \mathbf{c}}}$ (one for each norm $N \in \mathbf{N}$ ); the second partial derivatives for the $\left(\begin{array}{l}n \\ 2\end{array}\right)$ possible combinations of two norm nodes from $\mathbf{N}$; the third partial derivatives for the $\left(\begin{array}{l}n \\ 3\end{array}\right)$ possible combinations of three norm nodes from $\mathbf{N}$; and so on until the $n$-th partial derivative $\frac{\delta^{n} P\left(\mathbf{O}_{\text {true }} \mid \mathbf{c}\right)}{\delta \theta_{N 1_{\text {violl }}} \ldots \delta \theta_{N n_{\text {violl }}}}$.

For instance, in the case of $\mathbf{N}=\{N 1, N 2\}$, we have that $n=2$, $\operatorname{co}(\mathbf{N}, 1)=\{\{N 1\},\{N 2\}\}$, and $\operatorname{co}(\mathbf{N}, 2)=\{\{N 1, N 2\}\}$, and inequality (8) corresponds to inequality (9).

$$
\begin{aligned}
& P\left(\mathbf{O}_{\text {true }} \mid \mathbf{c}\right) \\
& +\frac{\delta P\left(\mathbf{O}_{\text {true }} \mid \mathbf{c}\right)}{\delta \theta_{N 1_{\text {viol } \mid \mathbf{c}}}} \cdot \Delta \theta_{N 1_{\text {viol } \mid \mathbf{c}}}+\frac{\delta P\left(\mathbf{O}_{\text {true }} \mid \mathbf{c}\right)}{\delta \theta_{N 2_{\text {viol } \mid \mathbf{c}}}} \cdot \Delta \theta_{N 2_{\text {viol } \mid \mathbf{c}}} \\
& +\frac{\delta^{2} P\left(\mathbf{O}_{\text {true }} \mid \mathbf{c}\right)}{\delta \theta_{N 1_{\text {viol } \mid \mathrm{c}}} \delta \theta_{N 2_{\text {viol] }}}} \cdot \Delta \theta_{N 1_{\text {viol } \mid \mathbf{c}}} \Delta \theta_{N 2_{\text {viol } / \mathrm{c}}} \geqslant t_{o a}
\end{aligned}
$$

The first partial derivatives in Eq. (8) can be computed as per Eq. (7), while the second partial derivative, in the case of two norms (as it is in Eq. (9)), can be computed as per Eq. (10).

$$
\begin{aligned}
& \frac{\delta^{2} P\left(\mathbf{O}_{\text {true }} \mid \mathbf{c}\right)}{\delta \theta_{N 1_{\text {viol } \mid \mathbf{c}}} \delta \theta_{N 2_{\text {viol } \mid \mathbf{c}}}} \\
& \quad=P\left(\mathbf{O}_{\text {true }} \mid N 1_{\text {viol }}, N 2_{\text {viol }}, \mathbf{c}\right)+P\left(\mathbf{O}_{\text {true }} \mid N 1_{o b}, N 2_{o b}, \mathbf{c}\right) \\
& \quad-P\left(\mathbf{O}_{\text {true }} \mid N 1_{\text {viol }}, N 2_{o b}, \mathbf{c}\right)-P\left(\mathbf{O}_{\text {true }} \mid N 1_{o b}, N 2_{\text {viol }}, \mathbf{c}\right)
\end{aligned}
$$

If we consider the running example from Fig. 3, the derivative in Eq. (10) can be computed as follows.

$$
\begin{aligned}
& P\left(\text { TripDur }_{\text {true }} \mid \text { SpdLim }_{\text {viol }}, \text { SafDst }_{\text {viol }}, \mathbf{c}\right) \cdot P\left(\text { Halted }_{\text {true }} \mid \text { SpdLim }_{\text {viol }}, \text { SafDst }_{\text {viol }}, \mathbf{c}\right)+ \\
& P\left(\text { TripDur }_{\text {true }} \mid \text { SpdLim }_{\text {ob }}, \text { SafDst }_{o b}, \mathbf{c}\right) \cdot P\left(\text { Halted }_{\text {true }} \mid \text { SpdLim }_{\text {ob }}, \text { SafDst }_{\text {ob }}, \mathbf{c}\right)- \\
& P\left(\text { TripDur }_{\text {true }} \mid \text { SpdLim }_{\text {viol }}, \text { SafDst }_{\text {ob }}, \mathbf{c}\right) \cdot P\left(\text { Halted }_{\text {true }} \mid \text { SpdLim }_{\text {viol }}, \text { SafDst }_{o b}, \mathbf{c}\right)- \\
& P\left(\text { TripDur }_{\text {true }} \mid \text { SpdLim }_{\text {ob }}, \text { SafDst }_{\text {viol }}, \mathbf{c}\right) \cdot P\left(\text { Halted }_{\text {true }} \mid \text { SpdLim }_{\text {ob }}, \text { SafDst }_{\text {viol }}, \mathbf{c}\right) .
\end{aligned}
$$

After determining the values of the opportune derivatives, as above reported, inequality (8) can be solved by solving the following optimization problem. 


$$
\begin{aligned}
& \text { minimize }_{\mathbf{x} \in \mathbb{R}^{n}} f(\mathbf{x}) \\
& \text { subject to: } t_{\text {oa }}-f(\mathbf{x}) \leqslant 0
\end{aligned}
$$

where $\mathbf{x}=\left(x_{1}, \ldots, x_{n}\right)$ is a vector of real values, such that $x_{i}$ is a possible value for $\Delta \theta_{N i_{\text {viollc }}}$ and $f(\mathbf{x})$ is the left member of inequality (8). Notice that the constraint to which the optimization problem is subject to corresponds to the canonical form of Eq. (8). Solving the optimization problem (11) means to determine the minimum values for the $n$ parameters $\Delta \theta_{N 1_{\text {viol] }}}, \ldots, \Delta \theta_{N n_{\text {violc }}}$ that satisfy the desired constraint of inequality (8) (i.e., the probability of achieving the objectives, after applying the required change in the probability of violating the enforced norms, is above the desired threshold $t_{o a}$ ).

Analogously to naive sensitivity analysis, $n-C P T$ sensitivity analysis suggests to change (increase or decrease) the violations of norms of the amount of the corresponding $R R S$ s determined by solving inequality (8). For instance, in the case of two norms, if $\Delta \theta_{N 1_{\text {violle }}}=+0.2$ and $\Delta \theta_{N 2_{\text {viol } \mathrm{c}}}=-0.5$, then the suggestion is to increase $P\left(N 1_{\text {viol }}\right)$ of 0.2 and to decrease $P\left(N 2_{\text {viol }}\right)$ of 0.5 . Differently from the previous strategy, however, such values are obtained taking into account the change applied at the same time to the probability of violating all norms (instead of applying a change only one norm at a time).

\subsubsection{Category-based strategies}

Category-based strategies classify norms into different categories, based on their exhibited norm violation and on their relationship with the system-level objectives discovered at runtime, and determine an adequate revision for each norm based on their category. We present two strategies of this type (Synergy $+M L E$ and State-based), based on two heuristic strategies presented in [23] and used to suggest a revision of regimented norms. In this paper we adapt them to support the revision of sanctions.

Synergy + MLE This strategy is based on the pureBN strategy presented in [23]. We distinguish between norms that are more useful when obeyed (useful-ob for brevity) or more useful when violated (useful-viol). Furthermore, norms can also be either most likely obeyed when the objectives are not achieved (likely-ob for brevity) or most likely violated (likely-viol). In order to distinguish between useful-ob and useful-viol we calculate the combined synergy $\mathbf{n}_{d}$ (as per Eq. (5)). Norms with value $o b$ in $\mathbf{n}_{d}$ are $u$ seful-ob, norms with value viol in $\mathbf{n}_{d}$ are useful-viol. In order to distinguish between likely-ob and likely-viol, instead, we determine the most likely explanation [36] $\mathbf{m l e}$ for $\mathbf{O}_{\text {false }}$ in context $\mathbf{c}$, as follows (with ov defined as per Eq. (5)).

$$
\mathbf{m l e}=\operatorname{argmax}_{\mathbf{n} \in \mathbf{o v}} P\left(\mathbf{n} \mid \mathbf{O}_{\text {false }} \wedge \mathbf{c}\right)
$$

Norms with value $o b$ in mle are likely-ob, norms with value viol in mle are likely-viol.

Synergy $+M L E$ suggests to increase violations of norms belonging to category usefulviol (more useful when violated); to reduce violations of norms belonging to both categories useful-ob and likely-viol (norms that are more useful when obeyed, but most likely violated when the objectives are not achieved); and to do nothing for, or reduce violations of, norms belonging to both categories useful-ob and likely-ob (norms that are more useful when obeyed, and most likely obeyed when the objectives are not achieved).

The original pureBN strategy [23] included the concept of harmful norm: a norm that is better when disabled. The suggestion of pureBN for harmful norms is to disable them. In this paper we only consider active norms and we focus on the sanction revision, thereby 
Fig. 5 System states (points) in four states (A-D) w.r.t. average norm obedience and objectives achievement probability

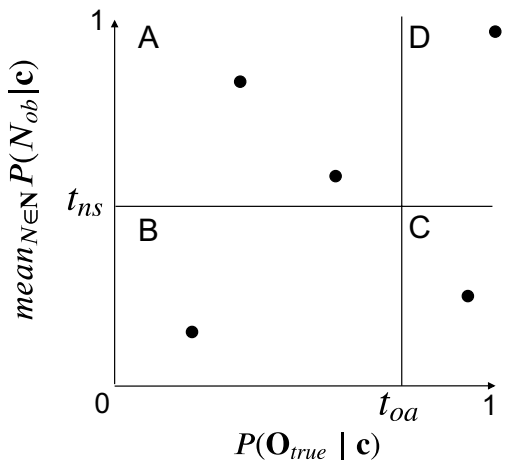

omitting specific suggestions for harmful norms. However, a suggestion of increasing violation of a norm $N$, may lead to enforce $N$ with a sanction equals to 0 . In this paper, enforcing a norm $N$ with a sanction of 0 corresponds to disabling $N$.

Finally, note that Synergy+MLE is a refinement of Combined synergy strategy. In addition to the combined synergy, this strategy also takes into account the most likely explanation for the objectives being not achieved, in terms of obedience or violation of norms.

State-based This strategy, based on the stateBased strategy presented in [23], considers, in addition to the classification of norms described for strategy Synergy $+M L E$, information about the system state in context c. In particular, as illustrated in Fig. 5, the system can be in four states with respect to the average norm obedience, calculated as the mean $n s=\operatorname{mean}_{N \in \mathbf{N}} P\left(N_{o b} \mid \mathbf{c}\right)$, and the objectives achievement probability oa $=P\left(\mathbf{O}_{\text {true }} \mid \mathbf{c}\right)$.

- In state A, norms are sufficiently obeyed, but this does not lead to sufficient objectives achievement (i.e., $n s \geqslant t_{n s}$ and $o a<t_{o a}$ for some given $t_{n s}$ and $t_{o a}$ ).

- In state B, norms are not sufficiently obeyed and also objectives are not achieved (i.e., $n s<t_{n s}$ and $\left.o a<t_{o a}\right)$.

- In state $\mathrm{C}$, the objectives are achieved even though the norms are not obeyed (i.e., $n s<t_{n s}$ and $\left.o a \geqslant t_{o a}\right)$.

- In state D, (the desired state of the system) the norms are satisfied and the objectives are achieved (i.e., $n s \geqslant t_{n s}$ and $\left.o a \geqslant t_{o a}\right)$.

If the system is in state A, State-based suggests to increase violations of norms belonging to both categories useful-viol and likely-ob, i.e., norms that are more useful when violated but most likely obeyed when the objectives are not achieved, if any. Otherwise, Statebased suggests to do nothing for (or to reduce violations of) the current norm set. In this case, there is probably some aspect of the system that has not been considered during its design, for the current norms are mostly obeyed and they are most useful when obeyed, but the system-level objectives are not achieved as desired. If the system is in state B, Statebased suggests to reduce violations of norms belonging to both categories useful-ob and likely-viol, i.e., norms that are more useful when obeyed but most likely violated when the objectives are not achieved. It also suggests to increase violations of norms belonging to category useful-viol, i.e., norms that are more useful when violated. If the system is in state $\mathrm{C}$, finally, State-based suggests to increase violations of norms belonging to both categories useful-viol and likely-viol, if any. Otherwise it suggests to decrease violations of norms belonging to both categories useful-ob and likely-viol. 
While Synergy+MLE suggests for all the norms in $\mathcal{N}$ the most adequate revision to perform, State-based considers the global state of the system and suggests to revise only a specific category of norms at every iteration (for the norms that do not belong to the category above mentioned it is suggested to do nothing). In case of high number of norms enforced, this strategy may significantly reduce the number of revisions that need to be performed at every step.

\subsection{Sanctions revision}

Consider a norm $N=(p, s)$ and a revision of it $N^{\prime}=\left(p, s^{\prime}\right)$, with $s^{\prime} \neq s$. Let $P\left(N_{\text {viol }} \mid \mathbf{c}\right)$ be $N$ 's exhibited norm violation (i.e., the probability of violating $N$ monitored during system's execution) in context c. We call applied revision strength the difference $U B\left(N_{\text {viol }}^{\prime}, \mathcal{N}\right)-P\left(N_{\text {viol }} \mid \mathbf{c}\right)$ between the upper bound $U B$ for violation of $N^{\prime}$ (as per Sect. 4.2) and the $N$ 's exhibited norm violation. For instance, in the example reported in Fig. 4, supposing that when enforcing $N_{1}=\left(s p_{50}, 1\right)$ and $N_{2}=\left(\right.$ dist $\left._{2}, 0\right)$ the exhibited norm violation of $N_{1}$ is 0.3 , the applied revision strength when revising $N_{1}$ into $N_{1}^{\prime}=\left(s p_{50}, 2\right)$ is $0-0.3=-0.3$, while the applied revision strength when revising $N_{1}$ into $N_{1}^{\prime}=\left(s p_{50}, 0\right)$ is $1-0.3=0.7$.

The strategies described in Sect. 5.1, provide, for each norm $N$ in $\mathcal{N}$, a suggestion such as reduce/increase violations of $N$, do nothing with $N$, reduce/increase violations of $N$ of a certain amount RRS (as per Sect. 5.1.2). Given these suggestions, and all possible sets of sanctions $\mathcal{S}$ that can be used to enforce norms in $\mathcal{N}$, we need to find a new set of sanctions such that the applied revision strength satisfies (as much as possible) the given suggestions.

A trivial solution is to systematically go through all elements in $\mathcal{S}$ until the desired sanction set (if it exists) is found. Such solution is however computationally expensive, as the number of possible sanction sets is $(\max (\mathcal{B})+2)^{n}$, with $\max (\mathcal{B})+2$ maximum budget among all agent types $(\max (\mathcal{B}))$ plus sanction 0 and sanction $\max (\mathcal{B})+1$, and $n$ number of norms.

In the following, we propose a simple alternative way to explore the search space $\mathcal{S}$ that can be used in case of a population of consistent agent types as per Definition 5 . With a population of consistent agent types, according to Proposition 3, the upper bound of the probability of violating norms decreases monotonically when any sanction increases. This means that given a sanction set, and the exhibited norm violation for each enforced norm, if we desire to apply a negative revision strength, we need to move towards higher values of sanctions. To apply a positive revision strength, instead, we could change in any way the sanctions (even though typically we should move towards lower values of sanctions), since the currently exhibited norm violation could be lower than the upper bound of norm violation with an higher sanction.

Under the assumption of consistent agent types, we can reduce therefore the exploration of $\mathcal{S}$ by directing the search towards the desired values of sanctions. For instance, suppose to have two norms $N 1=\left(p_{1}, s_{1}\right)$ and $N 2=\left(p_{2}, s_{2}\right)$, and a list of suggestions sugg $=$ (reduce, increase) for a context $\mathbf{c}$ (i.e., it is suggested to reduce violations of norm $N 1$ and to increase violations of norm $N 2$ in context $\mathbf{c})$. Given sugg, we need to look for a new sanction set $\left\{s_{1}^{\prime}, s_{2}^{\prime}\right\}$ such that $U B\left(N 1_{\text {viol }}^{\prime}, \mathcal{N}^{\prime}\right)<P\left(N 1_{\text {viol }} \mid \mathbf{c}\right)$ and $U B\left(N 2_{\text {viol }}^{\prime}, \mathcal{N}\right)>P\left(N 2_{\text {viol }} \mid \mathbf{c}\right)$, with $\mathcal{N}^{\prime}=\left\langle N 1^{\prime}, N 2^{\prime}\right\rangle$. We can therefore reduce the search space to the subset of $\mathcal{S}$ such that $s_{1}^{\prime} \geqslant s_{1}$ and $s_{2}^{\prime} \neq s_{2}$.

Algorithm 1 reports the pseudo-code of a procedure to perform such search. 
Notice that if preferences are not consistent, we have no guarantees that by moving towards higher values of sanctions we will not increase violations of norms, since Proposition 3 does not hold in the general case (i.e., for preferences that are not consistent). Despite this, one may still heuristically explore $\mathcal{S}$ by using Algorithm 1, also when not all preferences are consistent.

Algorithm 1 is invoked when a suggestion of norm revision has been determined with one of the strategies of Sect. 5.1 after a norm revision is triggered, and there is at least one sanction set that has not been tried previously in context $\mathbf{c}$. If a sanction set has already been tried, we know it is not effective (otherwise no further norm revision would have been triggered). If all possible sanction sets have been already tried (omitted from Algorithm 1), then the sanction set that, when enforced, maximizes $P\left(\mathbf{O}_{\text {true }} \mid \mathbf{c}\right)$ is selected.

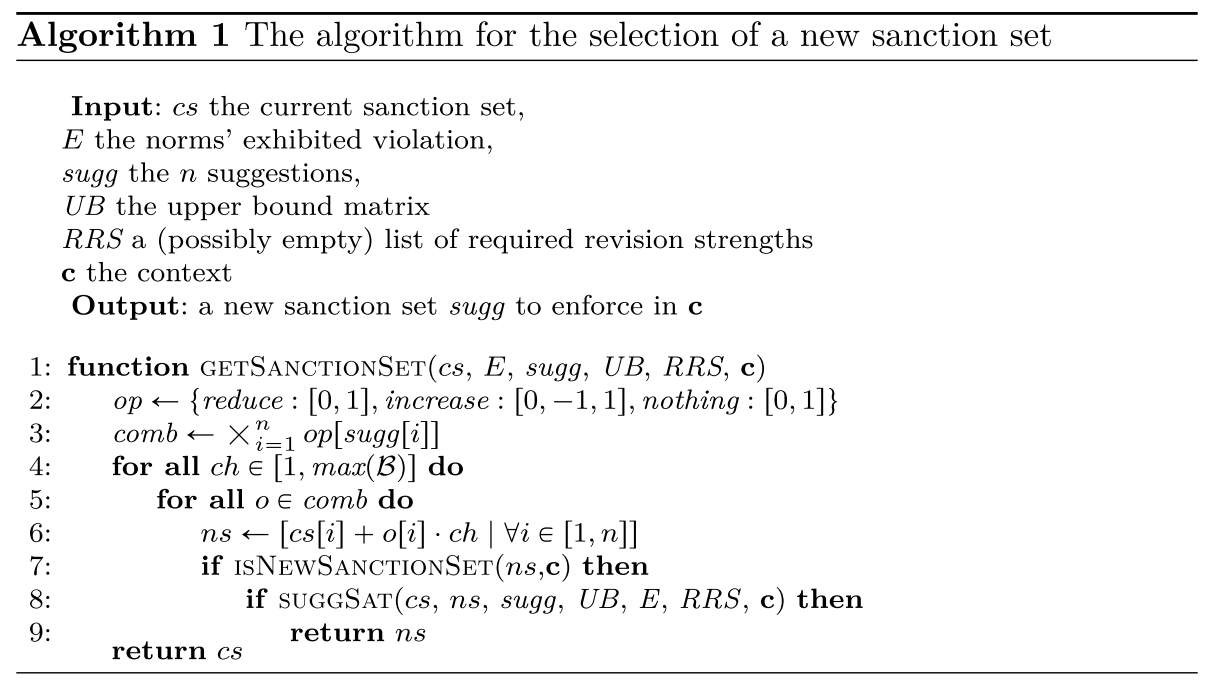

The algorithm takes as input: the list of currently enforced sanctions $c s$; the exhibited violation of the enforced norms $E$; the list sugg of suggestions obtained with one of the strategies of Sect. 5.1 (a value reduce (or increase, or nothing) in sugg[i] corresponds to a suggestion to reduce (increase, or do nothing with) violations of the $i$-th norm); a matrix $U B$ containing the upper bounds for norms violations as per Fig. 4; a list $R R S$ of required revision strengths (empty if no sensitivity-based strategy is used); and the context c. As output, Algorithm 1 returns a (possibly new) list of sanctions to use to enforce norms in context $\mathbf{c}$.

The algorithm explores the possible sanction sets starting from the current sanction set $c s$. The first step is to determine the subset of $\mathcal{S}$ to explore. Notice that, if a reduce suggestion has been given for norm $i$ (i.e., sugg $[i]=$ reduce), the new sanction for norm $i$ must be greater of equal than the current one (i.e., $n s[i] \geqslant c s[i])$. This means that $n s[i]$ has to be equal to $c s[i]+c h$, with $c h \geqslant 0$ amount of change. Conversely, if sugg $[i]=$ increase, $n s[i]$ has to be equal to either $c s[i]+c h$ or $c s[i]-c h$. If we put these cases together (line 6 of Algorithm 1), $n s[i]$ has to be equal to $c s[i]+o[i] \cdot c h$, with $o[i] \in\{0,1\}$ if $\operatorname{sugg}[i]=$ reduce, and $o[i] \in\{0,-1,1\}$ if sugg $[i]=$ increase, and $c h>0$ amount of change. 
Variable comb (line 3) is a set of all possible combinations of operators $o[i]$ for each norm $i$, obtained from their suggestion sugg[i] (see op[sugg[i]], which retrieves from the labeled set $o p$ declared at line 2, the opportune list of operators given suggestion $\operatorname{sugg}[i])$. For instance, supposing to have two norms $N 1=\left(p_{1}, s_{1}\right)$ and $N 2=\left(p_{2}, s_{2}\right)$, and a list of suggestions sugg $=$ (reduce, increase), we have that $\operatorname{op}[\operatorname{sugg}[1]]=[0,1]$ and op $[\operatorname{sugg}[2]]=[0,-1,1]$ and comb is the set of all possible combinations of operators in $o p[\operatorname{sugg}[1]]$ and $o p[\operatorname{sugg}[21]]$, i.e., $\{(0,0),(0,-1),(0,1),(1,0),(1,-1),(1,1)\}$, such that given a certain element $o \in c o m b, o[i]$ is the operator to apply to the change of sanction $c s[i]$.

The algorithm iterates through all possible changes that can be applied to sanctions (line 4). For each possible change, the algorithm iterates through all possible new sanction sets that can be obtained with the combinations of operators in comb (lines 5-6). Notice that, by iteratively increasing the change, we explore the search space at increasing distance from the current sanction set. This means that if the algorithm finds a new (function isNewSanctionSet at line 7) sanction set $n s$ that satisfies the given suggestions (function suggSat at line 8), such sanction set is also the closest possible to the current one.

Finally, if no new sanction set satisfying the suggestions is found, the current sanction set is returned. In this case, in our framework a random sanction set never tried before is enforced in context $\mathbf{c}$.

Notice also that function suggSat (line 8), whose purpose is to verify that a proposed sanction set satisfies the given suggestions, does not need to require that all suggestions are perfectly satisfied. In particular, especially when suggestions include also a required revision strength (i.e., when using sensitivity-based strategies), it may be more useful to search for a good-enough sanction set. For our experiments, described in Sect. 6, when list RRS is not empty, we keep track of the best new sanction set found so far (if not all suggestions are satisfied) and for every new sanction set tested we require at least $80 \%$ of suggestions to be satisfied. Furthermore in case of suggestion nothing, since unlikely, in our experiments, that the exhibited probability of a norm exactly corresponds to a value on its upper bound, we accept also a reduction of the probability of violating the norm of a small $\epsilon$ (we used $\epsilon=0.1$ ).

After enforcing the new norm set $\mathcal{N}$, obtained by revising the sanctions of norms in $\mathcal{N}$ according to the new sanction set obtained from Algorithm 1, we monitor the new behavior of the agents and detect the new exhibited norm violation $P\left(N_{\text {viol }}^{\prime} \mid \mathbf{c}\right)$, for each norm $N^{\prime} \in \mathcal{N}^{\prime}$. We call actual revision strength the difference $P\left(N_{\text {viol }}^{\prime} \mid \mathbf{c}\right)-P\left(N_{\text {viol }} \mid \mathbf{c}\right)$ between the exhibited norm violation of $N^{\prime}$ and $N$, with $N^{\prime}=\left(p, s^{\prime}\right)$ and $N=(p, s)$.

\section{Experimentation}

We report on an experiment that investigates the process through which the normbased supervision mechanism of Sect. 4 identifies an optimal system configuration. The object of our study consists of the strategies for norm revision proposed in Sect. 5. In particular, we study the process through which the norm-based supervision mechanism identifies an optimal system configuration when employing each of the six proposed strategies as possible informed heuristics for defining the neighborhood of a 
configuration, i.e., the configurations where the sanctions of the enforced norms are revised as suggested by the heuristics.

We compare the results in terms of convergence speed. The convergence speed measures the number of steps (i.e., revisions of the sanctions of norms triggered) required by the heuristic strategies to make the norms effective in achieving the system-level objectives. This allows us to study the time efficiency of the norm revision strategies in refining sub-optimal norms at runtime.

\subsection{Experimental setting}

Our experiment is run through a simulation ${ }^{5}$ of the ring road scenario described in Sect. 3. Our implementation of the norm-based supervision mechanism of Sect. 4, as a modified version of hill climbing, is called SASS (Supervisor of Autonomous Software Systems). ${ }^{6}$ The supervisor performs a local search and stops when either (i) all the system configurations have been tried; or (ii) a local optimum (system configuration) is found that has objectives achievement probability $o a=P\left(\mathbf{O}_{\text {true }}\right)$ above the desired threshold $t_{o a}$. The objectives achievement probability of a certain system configuration is not known to SASS before the configuration is actually enforced. Such probability is determined at runtime from simulation data, given the chosen system configuration. In this experimental setting, the last system configuration that is selected before stopping is called optimal, since either the objectives achievement is above the desired threshold or there is no other better configuration.

In the ring road scenario, we consider the two contextual variables Vehicle density, which can be low (40 cars on the ring road) or high (80 cars) ${ }^{7}$ and Obstacle, which is true when an obstacle is placed on the outer lane of the ring road. Each car in the simulation is an agent that acts according to its specific characteristics, beliefs and preferences. At each simulation step, every agent also deliberates about a number of things, including its desired speed and the minimum safety distance, whether and how much to accelerate or decelerate, whether to change lane to surpass or to move back to the outer lane, whether to activate the turn signals. Agents' decisions are based on their own internals, which are specific for each agent and unknown to the norm revision mechanism. In our simulations, when an agent equally prefers two alternatives $x$ and $y$ concerning the speed and safety distance (i.e., $x \sim y$ ), the agent applies a deterministic choice to determine what state of affair to pursue (i.e., simply the first one in the representation of the alternatives), instead of random choice.

In order to define norms and agents' preferences, we consider the set of propositional atoms $L=\left\{s p_{1} 5, s p_{8}, s p_{3}\right.$, dist $_{0.5}$, dist $\left._{1}, s p_{2}\right\}$, with $A L=\left(L_{1}, L_{2}\right)$ and $L_{1}=\left\{s p_{1} 5, s p_{8}, s p_{3},\right\}$ and $L_{2}=\left\{\right.$ dist $_{0.5}$, dist $t_{1}$, dist $\left.t_{2}\right\}$. Each element in $L_{1}$ represents a speed in $\mathrm{m} / \mathrm{s}$ and each element in $L_{2}$ represents a safety distance in meters. Furthermore, we consider a language $\mathcal{B}=\{0,1,2\}$ for defining budgets and a language $\mathcal{S}=\{0,1,2,3\}$ for defining sanctions of norms.

\footnotetext{
${ }^{5}$ For our experiment, we used the SUMO traffic simulator [35] and CrowdNav+RTX [43].

${ }^{6}$ The source code and the material for experiments' replication can be found in [25].

7 The density values have been determined empirically based on the size of the ringroad used for the experiments.
} 


\subsubsection{Agent types}

We experiment with four types of rational agents with consistent preferences (as per Definition 5). In the following we briefly describe such types, and we report in "Appendix 2" the full preferences.

- BraveRich is a consistent basic preference that adheres to Definition 2b, i.e., where alternatives are ordered by propositional atom. It describes an agent type with a maximum budget of 4 , that prefers to drive fast and to keep a short safety distance, and that gives priority to the short safety distance rather than to driving fast.

- BraveMiddleClass is a consistent preference composed by two basic preferences. The first basic preference $\left(A_{1}, \geq_{1}\right)$ adheres to Definition $2 \mathrm{~b}$. The alternatives in $A_{1}$ are such that $A_{1}=\left\{\left(\left\langle p_{1}, b_{1}\right\rangle, \ldots,\left\langle p_{n}, b_{n}\right\rangle\right) \mid p_{i} \in L_{i} \&\left(b_{1}, \ldots, b_{n}\right) \in B L_{1}\right\}$, with $B L_{1}=\{(0,0),(0,1),(1,0),(1,1)\}$. The second basic preference $\left(A_{2}, \geq_{2}\right)$ adheres to Definition 2a. The alternatives in $A_{2}$ are such that $\quad A_{2}=\left\{\left(\left\langle p_{1}, b_{1}\right\rangle, \ldots,\left\langle p_{n}, b_{n}\right\rangle\right) \mid p_{i} \in L_{i} \&\left(b_{1}, \ldots, b_{n}\right) \in B L_{2}\right\}, \quad$ with $B L_{2}=\{(0,2),(1,2),(2,2),(2,1),(2,0)\}$. BraveMiddleClass describes an agent type similar to BraveRich, but that is willing to pay no more than 2 for a certain state of affairs. The alternatives in $A_{2}$ are ordered by required budget and, for consistency, they maintain the same relative order as in $A_{1}$.

- BravePoor is a consistent basic preference ordered by required budget, as per Definition 2a. It describes an agent type that equally prefers to drive fast or slow and to keep a short or long safety distance, but is not willing to pay anything to reach any state of affairs.

- Cautious is a consistent basic preference ordered by required budget, as per Definition 2a. It describes an agent type that equally prefers to drive slow or fast and to keep a long or short safety distance, and is not willing to pay anything to reach any state of affairs. Notice that this preference is equivalent to BravePoor, however due to the deterministic mechanism of choice of an alternative that our agents employ (i.e., the first one in the representation of the alternatives), these two agent types will exhibit different behaviors at runtime. For instance, even though states of affairs where $s p_{1} 5$ and dist $t_{0.5}$ hold are equally preferred to state of affairs where $s p_{3}$ and $d i s t_{0.5}$ hold, in both preferences, and they could both be chosen in the case of random choice, in our simulation, given enough budget, BravePoor will aim at a state of affair where $s p_{1} 5$ and dist ${ }_{0.5}$ hold, while Cautious will aim at a state of affair where $s p_{3}$ and $d i s t_{0.5}$ hold.

We consider three distributions of types of agents:

- uniform the entire population of agents is uniformly distributed across the four types above described.

- mostly compliant $75 \%$ of agents belongs to type Cautious and the rest is uniformly distributed across the remaining types.

- mostly violating 75\% of agents belongs to type BraveRich and the rest is uniformly distributed across the remaining types.

Note that despite our estimation of the preferences of the agents concerning speed and safety distance, we do not have any control on the exact speed or safety distance of the 


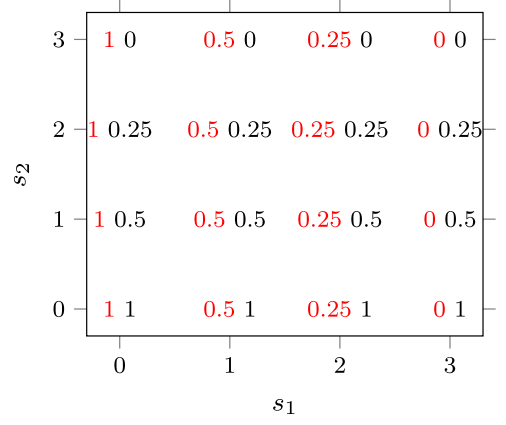

(a) uniform

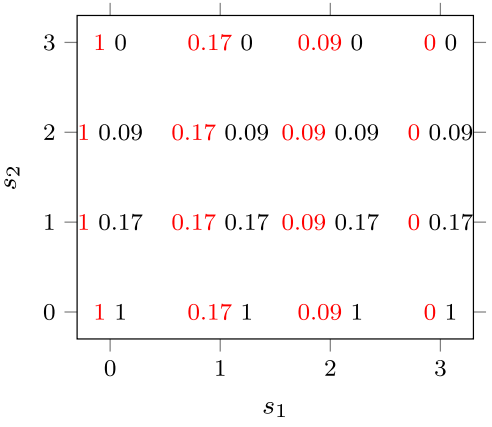

(b) mostly compliant

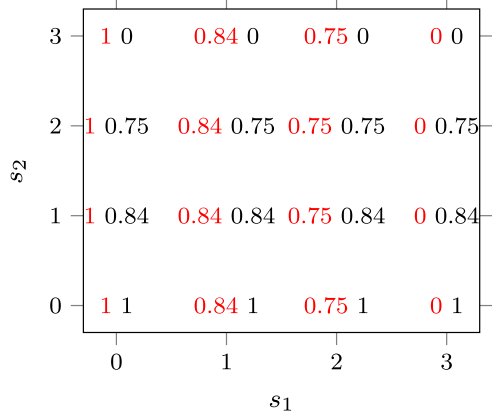

(c) mostly violating

Fig. 6 Upper bound of the probability of violating norms $\operatorname{SpdLim}_{x}=\left(s p_{x}, s_{1}\right)$ (red) and $\operatorname{SafDst}_{y}=\left(\right.$ dist $\left._{y}, s_{2}\right)$ (black) with different agent type distributions. In each subfigure the X-axis represents the sanction $s_{1}$ of norm SpdLim ${ }_{x}$, while the y-axis represents the sanction $s_{2}$ of norm SafDst

agents, which is internally and opaquely set by the agents, together with the rest of their behaviors.

\subsubsection{Norms}

We consider four ordered norm sets: $\mathcal{N}_{31}=\left\langle\operatorname{SpdLim}_{3}, \operatorname{SafDst}_{1}\right\rangle, \mathcal{N}_{32}=\left\langle\operatorname{SpdLim}_{3}\right.$, SafDst $\left._{2}\right\rangle$, $\mathcal{N}_{81}=\left\langle\operatorname{SpdLim}_{8}\right.$, SafDst $\left._{1}\right\rangle, \quad \mathcal{N}_{82}=\left\langle\operatorname{SpdLim}_{8}\right.$, SafDst $\left._{2}\right\rangle, \quad$ with $\quad \operatorname{SpdLim}_{x}=\left(s_{x}, s_{1}\right)$, SafDst $_{y}=\left(\right.$ dist $\left._{y}, s_{2}\right), x \in\{3,8\}, y \in\{1,2\}$ and $s_{1}$ and $s_{2}$ sanctions in $\mathcal{S}$.

Figure 6 illustrates the upper bounds of the probability of violating the two norms SpdLim $_{x}$ and SafDst $t_{y}$ above defined (as per Sect. 4.2) for the three agent type distributions.

Notice that the reported upper bounds hold for all combinations of the values $x$ and $y$ above defined (i.e., values of speed limit and minimum safety distance). This is due to the types of agents that we considered for our experiments. BraveRich prefers to keep a speed of $15 \mathrm{~m} / \mathrm{s}$ by maintaining a short safety distance and it is willing to pay a sanction of 2 for each of these aspects. When the sanction of a norm is above 2 this agent is compliant with the norm, regardless of the value of the speed limit, because the agent has no budget for violating the norm. BraveMiddleClass is analogous to BraveRich but with a maximum budget of 1 for the violation of a norm: up to sanction 1 BraveMiddleClass has reason 
to violate a norm (it also prefers to go at a speed of $15 \mathrm{~m} / \mathrm{s}$ by maintaining a short safety distance), while when the sanction of a norm is above 1 BraveMiddleClass is compliant. Finally BravePoor and Cautious have reason to violate the norms only when their sanctions is 0 . With higher sanctions, these agent types are compliant.

Furthermore notice that, since all the agent types that we considered are consistent as per Definition 5, the upper bounds reported in Fig. 6 satisfy Proposition 3: when increasing the sanction of only one norm the upper bound of violating the other norm never increases. This allows us to take advantage, in our experiments, of Algorithm 1 for the selection of a new sanction set.

\subsection{Experiments}

By combining the three distributions of agents of Sect. 6.1.1 with the four norm sets of Sect. 6.1.2, we derived 12 different experiments. We ran a simulation of the ring road for each of the 12 experiments and we collected data about norm obedience and objective achievement in the four different operating contexts $\mathbf{c} 1=$ VehicleDensity $_{\text {low }} \wedge$ Obstacle $_{\text {false }}$, c2 $=$ VehicleDensity $_{\text {low }} \wedge$ Obstacle $_{\text {true }}, \quad \quad \mathbf{c} 3=$ VehicleDensity $_{\text {high }} \wedge$ Obstacle $_{\text {false }}$, $\mathbf{c} 4=$ VehicleDensity $_{\text {high }} \wedge$ Obstacle $_{\text {true }}$. This means that during a simulation, the contexts in which the cars on the ring road operate changes three times (for a total of fours different operating contexts in each simulation). During the simulations, we monitored the behavior of the cars and sanctioned each car that violated one of the enforced norms. A car sanctioned for the violation of a norm $N$ was not sanctioned anymore for violations of norm $N$ until it completed a full loop of the ring road. The Boolean value of the system-level objectives was measured every 25 simulation steps. The objective TripDur was considered achieved if, on average in the 25 steps, the cars on the ring road took less than 2.5 times the theoretical average trip time ${ }^{8}$ to complete a loop of the ring road. The objective Halted was considered achieved if, on average in the 25 steps, less than $x \%$ of cars were halted on the ring road, with $x=25$ if the density of vehicles on the ring road is high, and $x=5$ if the density of vehicles is low. ${ }^{9}$ A car in SUMO is considered halted if its speed is below 0.1 $\mathrm{m} / \mathrm{s}$. Cars could be halted on the ring road for several reasons. For example, the presence of an obstacle may force them to stop and wait for the right moment to surpass the obstacle or breaking waves may force cars to temporary slow down significantly to avoid collisions.

In every experiment that we perform, the system has $n^{m}$ possible configurations, with $n$ possible sanction sets and $m$ different operating contexts. Since the speed of convergence to an optimal solution depends on the initial system configuration (i.e., a different amount of revisions may be required starting from different initial configurations), we execute each strategy starting from each possible configuration and we calculate statistics information (i.e., median, maximum, mean and standard deviation) concerning the convergence speed in the different executions. To keep our experimentation's time manageable, in our experiments we considered only 2 of the 4 operating contexts: $\mathbf{c 2}$ and $\mathbf{c 3}$. This allowed us to reduce the number of possible configurations from $16^{4}$ to $16^{2}=256$ : 16 possible sanction sets for the enforced norms in any of the 2

\footnotetext{
8 The theoretical trip time is $\sum_{t_{i} \in \mathcal{T}} d_{i} \times t_{i, \mathcal{N}}$, with $\mathcal{T}$ being the set of agent types, $d_{i}$ being the percentage of agents of type $t_{i}$, and $t_{i, \mathcal{N}}$ being the theoretical time needed by $t_{i}$ to complete a loop in case of free ring road when norm set $\mathcal{N}$ is enforced.

9 Values for the evaluation of the objectives were determined based on some preliminary experimentation with the ring road simulation in order to retrieve a variegate set of experiments.
} 

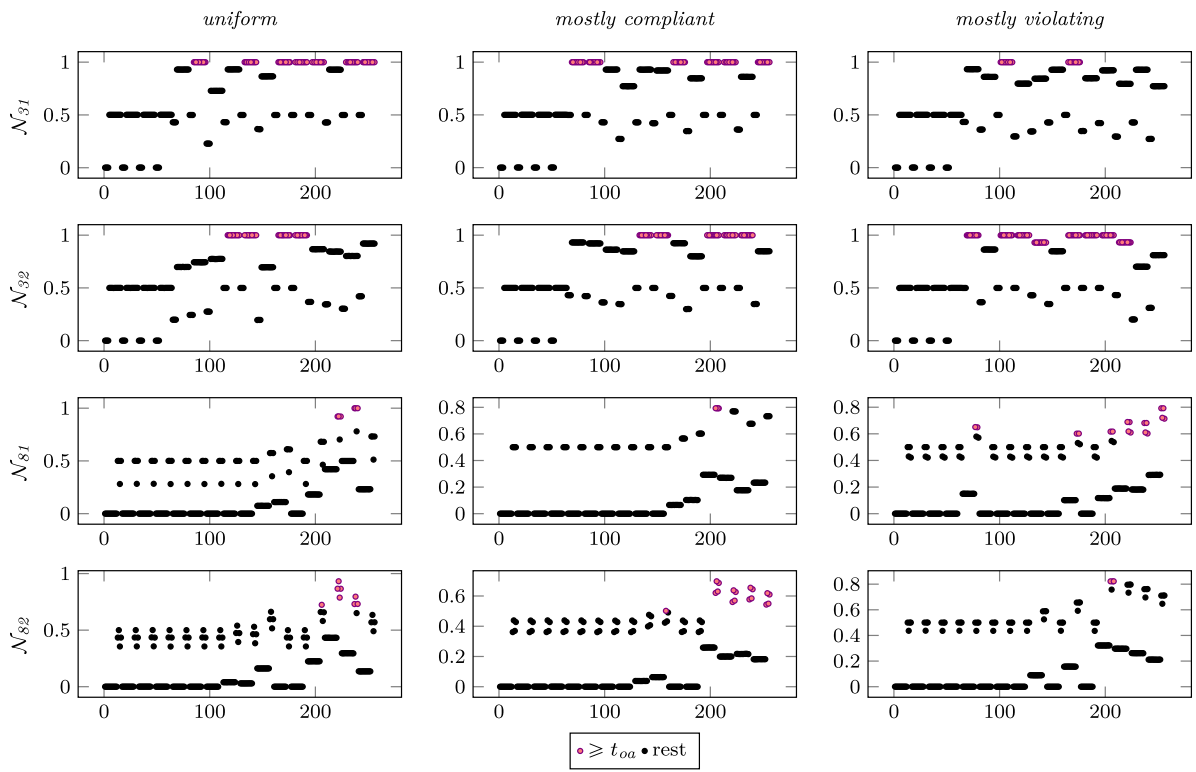

Fig. 7 Probability of objectives achievement (y-axis) for the 256 tried configurations (x-axis) in the 12 experiments

Table 2 The setting of the 12 experiments

\begin{tabular}{|c|c|c|c|c|c|c|}
\hline Exp. & ID & Agents distribution & Norm set & Contexts & $t_{o a}$ & Optimal \\
\hline 1 & DUN31 & uniform & $\mathcal{N}_{31}$ & $\mathbf{c} 2, \mathbf{c} 3$ & 0.99 & $84 / 256(32.8 \%)$ \\
\hline 2 & DUN32 & uniform & $\mathcal{N}_{32}$ & $\mathbf{c} 2, \mathbf{c} 3$ & 0.99 & $48 / 256(18.7 \%)$ \\
\hline 3 & DUN81 & uniform & $\mathcal{N}_{81}$ & $\mathbf{c} 2, \mathbf{c} 3$ & 0.9 & $6 / 256(2.3 \%)$ \\
\hline 4 & DUN82 & uniform & $\mathcal{N}_{82}$ & $\mathbf{c} 2, \mathbf{c} 3$ & 0.7 & $8 / 256(3.1 \%)$ \\
\hline 5 & DCN31 & mostly compliant & $\mathcal{N}_{31}$ & $\mathbf{c} 2, \mathbf{c} 3$ & 0.99 & $72 / 256(28.1 \%)$ \\
\hline 6 & $D C N 32$ & mostly compliant & $\mathcal{N}_{32}$ & $\mathbf{c} 2, \mathbf{c} 3$ & 0.99 & $60 / 256(23.4 \%)$ \\
\hline 7 & DCN81 & mostly compliant & $\mathcal{N}_{81}$ & $\mathbf{c} 2, \mathbf{c} 3$ & 0.79 & $4 / 256(1.6 \%)$ \\
\hline 8 & DCN82 & mostly compliant & $\mathcal{N}_{82}$ & $\mathbf{c} 2, \mathbf{c} 3$ & 0.5 & $17 / 256(6.6 \%)$ \\
\hline 9 & $D V N 31$ & mostly violating & $\mathcal{N}_{31}$ & $\mathbf{c} 2, \mathbf{c} 3$ & 0.99 & $24 / 256(9.4 \%)$ \\
\hline 10 & $D V N 32$ & mostly violating & $\mathcal{N}_{32}$ & $\mathbf{c} 2, \mathbf{c} 3$ & 0.9 & $96 / 256(37.5 \%)$ \\
\hline 11 & $D V N 81$ & mostly violating & $\mathcal{N}_{81}$ & $\mathbf{c} 2, \mathbf{c} 3$ & 0.6 & $18 / 256(7 \%)$ \\
\hline 12 & $D V N 82$ & mostly violating & $\mathcal{N}_{82}$ & $\mathbf{c} 2, \mathbf{c} 3$ & 0.8 & $3 / 256(1.2 \%)$ \\
\hline
\end{tabular}

contexts. Figure 7 shows the probability $P\left(\mathbf{O}_{\text {true }}\right)$ obtained with the 256 configurations in each of the 12 experiments and highlights the optimal configurations (the configurations s.t. $\left.P\left(\mathbf{O}_{\text {true }}\right) \geqslant t_{\text {oa }}\right)$. Every dot in Fig. 7 represents the probability of achieving the objectives during a simulation with a certain system configuration (i.e., $P\left(\mathbf{O}_{\text {true }}\right)$ ), considering both the contexts $\mathbf{c 2}$ and $\mathbf{c 3}$. In each sub-figure (one per experiment) we see therefore 256 dots, one per system configuration. Notice that in the 12 experiments, 


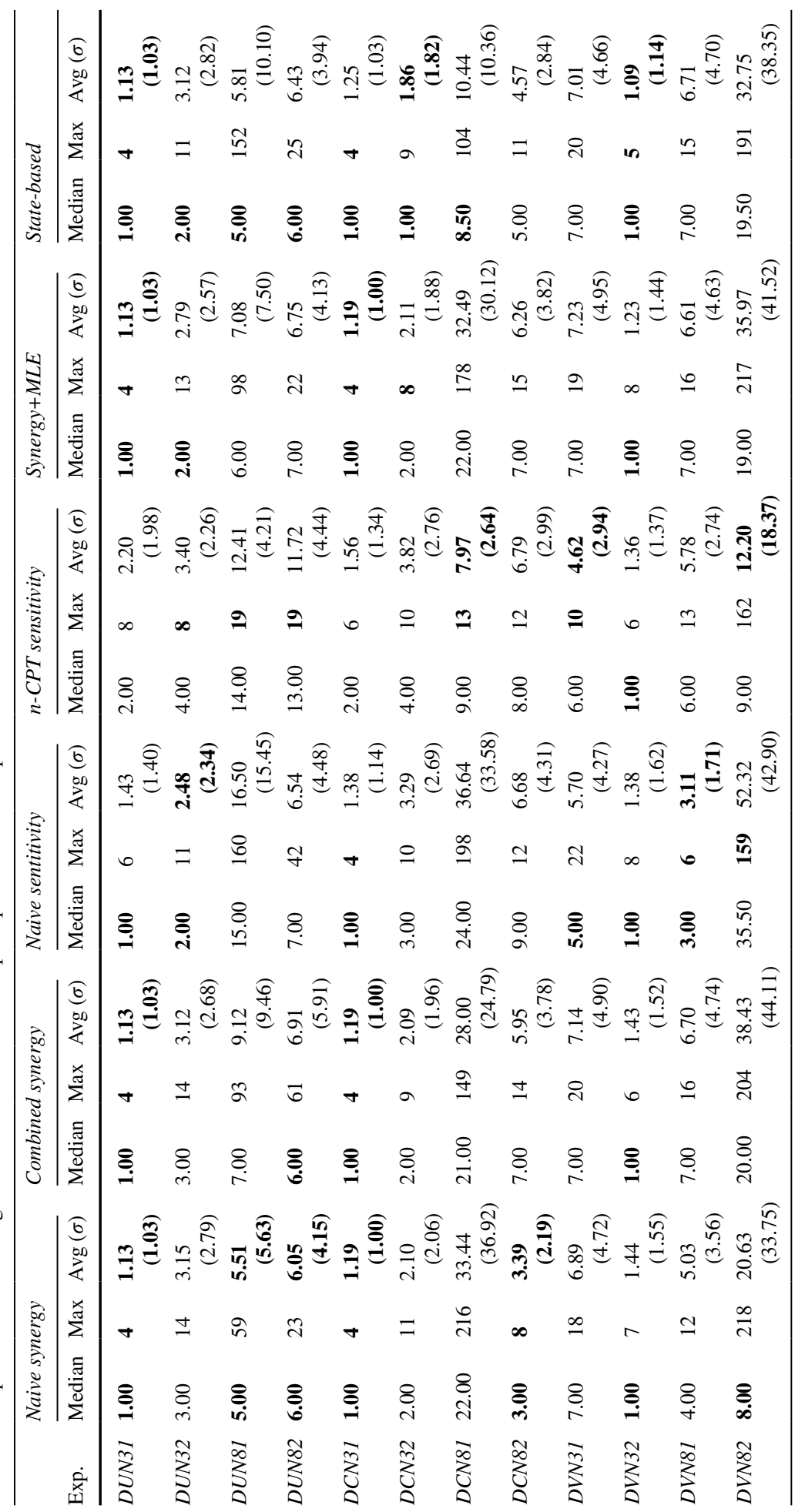


the distribution of the 256 configurations w.r.t. the probability of achieving the system-level objectives is different. In other words, a certain system configuration $c$ (i.e., enforcing norms with certain sanctions in the two contexts $\mathbf{c 2}$ and $\mathbf{c 3}$ ) can be effective in an experiment but ineffective in another experiment. This makes the 12 experiments independent, thereby increasing the generality of our results.

For each of the 12 experiments, we defined a different $t_{\text {oa }}$ as indicated in Table 2, which summarizes the entire experimental setting. The different thresholds allow us to test our strategies with different degrees of difficulty (i.e., number of optimal configurations to be found).

\subsection{Analysis of the results}

Table 3 reports the results concerning the steps required by the supervision mechanism to find an optimal configuration in the 12 experiments when employing each of the six proposed revision strategies. In particular, we report the median, the maximum, the average, and the standard deviation of the number of steps. We highlight in bold the values of the best performing strategies in each experiment.

On average, all the strategies required a limited number of steps to find an optimal configuration in almost all experiments. In the 12 experiments, while the number of optimal configurations to be found ranges from 3 to 96 out of 256 configurations, on average the strategies never required more than 52 steps to find one of those configurations (see columns Avg ( $\sigma$ ) in Table 3, where $\sigma$ is the standard deviation), with a minimum of 0 for all strategies (trivially in the cases the initial configuration is optimal, not reported in Table 3), a maximum of 218 in the most difficult scenario (see columns Max of experiment DVN82), and a median value never above 35 steps.

If we look at the average values, the strategy that performed less well in the 12 experiments is Naive sensitivity analysis, which, in order to find an optimal configuration among the 256 possible configurations, required an average number of steps between 1 and 52 . The strategy that, on average, performed best, instead, is $n$ - $C P T$ sensitivity analysis, requiring an average number of steps between 2 and 12. In particular, these results show that when using $n-C P T$ sensitivity analysis, on average, about 6 norm revisions were triggered by the norm-based supervision mechanism before finding a configuration where the system-level objectives were achieved as desired.

Despite $n$-CPT sensitivity analysis performed, on average, better than the other strategies in the 12 experiments, the results show that using that strategy was mostly advantageous when very few configurations were optimal among all the possible ones. In particular, $n$-CPT sensitivity analysis appeared to be more effective than the other strategies when the number of optimal configurations was lower than $2 \%$ of all the configurations. For instance, in experiment DCN81 (1.6\% of configuration are optimal), never more than 13 steps were required to find an optimal configuration when employing $n$-CPT sensitivity analysis, while the other strategies required a maximum number of steps between 104 and 216. Furthermore, while the median number of step is 9 with $n$-CPT sensitivity analysis, the median number of steps with the other strategies is more than twice. One exception is State-based, which in such experiment required an average number of steps similar to $n$-CPT sensitivity analysis and an even lower median. State-based, however, exhibited an higher variance, requiring in some executions up to 104 steps. In experiment DVN82 $(1.2 \%$ of configuration are optimal), while all other strategies (including State-based) required an 
$\square$ Naive synergy $\square$ Combined synergy $\square$ Naive sensitivity analysis n-CPT sensitivity analysis Synergy $+M L E$ State-based

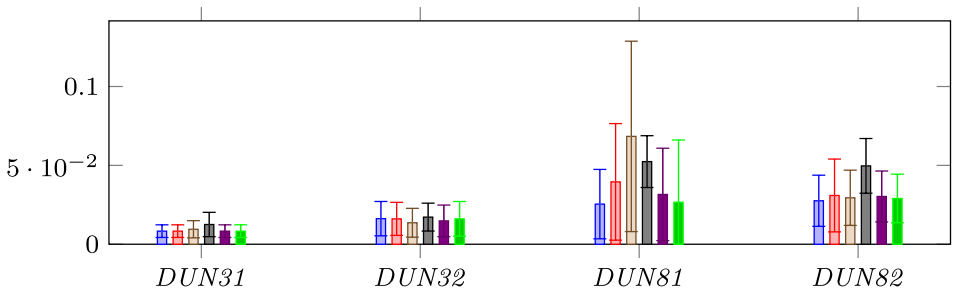

(a) uniform

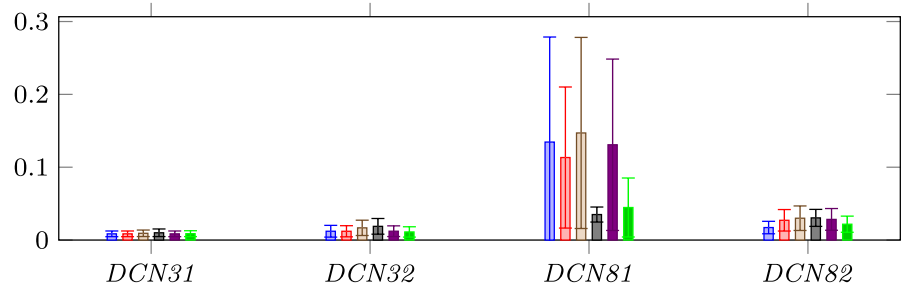

(b) mostly compliant

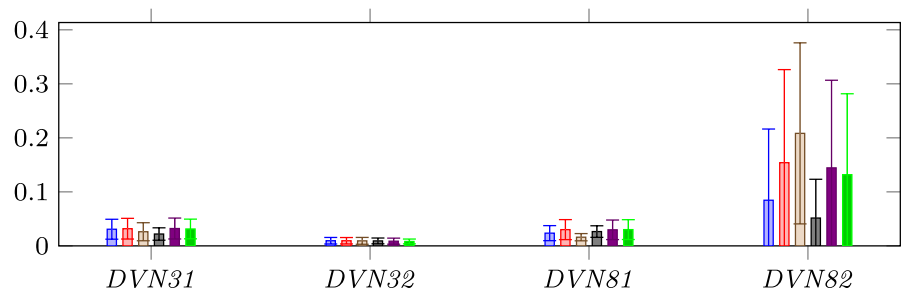

(c) mostly violating

Fig. 8 Average percentage of explored configurations before finding an optimal one

average number of steps between 20 and 52,n-CPT sensitivity analysis was able to find on average an optimal configuration in about 12 steps.

If we consider, instead, simpler experiments (e.g., DUN31 or DCN31), $n$-CPT sensitivity analysis did not outperform significantly the other strategies. In fact, if we consider the average number of steps, among all the strategies, Naive synergy outperformed (even though by few steps) all the others in 5 experiments, requiring in all of them less then 6 steps to find an optimal configuration. Furthermore in 8 experiments the average number of steps required by Naive synergy was below the average between the different algorithms. State-based had similar performances to Naive synergy and, even though it was the absolute best strategy in only 3 experiments in terms of average number of steps, in 8 experiments out of 12 it exhibited the lowest median value.

Figure 8 plots the percentage of configurations explored in the 12 experiments by the six strategies before finding an optimal one. In most experiments, all algorithms required to explore less than $10 \%$ of all configurations. The only cases that required to 


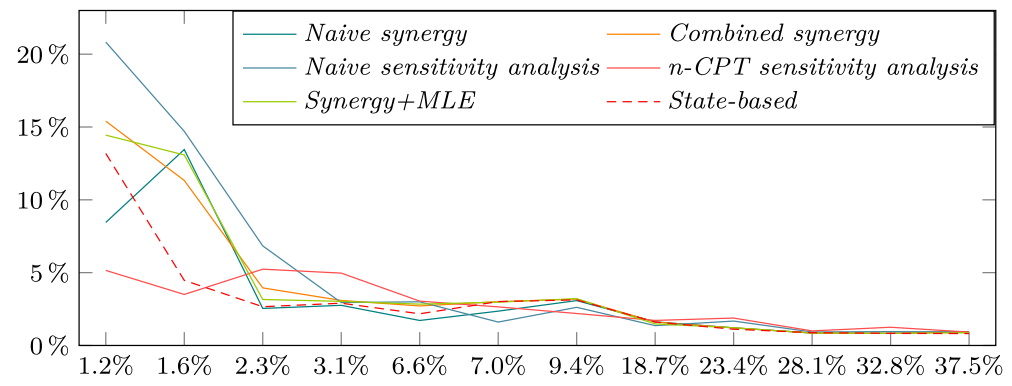

Fig. 9 Average percentage of explored configurations (y-axis) compared to the percentage of optimal configurations in the 12 different experiments (x-axis)

explore more than $10 \%$ of configurations were experiments $D C N 81$ and $D V N 82$, where the number of optimal configuration to be found was less than $2 \%$. Figure 8 emphasizes that all proposed strategies performed similarly, with the exception of $n$-CPT sensitivity analysis, which did not show a degradation in the cases of very few optimal configurations and required to explore a significantly lower number of configurations.

The values in Table 3 and in Fig. 8 concern the absolute number of steps required, and configurations explored, to find one of the optimal configurations among the total amount of 256 configurations. They provide an overview of the behaviour of the strategies proposed in this paper in problems of different difficulty with a search space of 256 possible solutions. Figure 9 compares the percentage of explored configurations by the different strategies with the percentage of optimal configurations to be found.

Note that, in problems with more than $6 \%$ of optimal configuration, the strategies did not exhibit significant differences. In more difficult problems (less than $3 \%$ of optimal configurations), the number of configurations to explore increased up to $20 \%$ with Synergy $+M L E$, Combined synergy and in particular with Naive sensitivity analysis. Naive synergy and State-based, instead, as reported above, exhibited a similar behavior in most of the cases. In problems with less than $2 \%$ of optimal configurations, however, they also required to explore a higher number (up to $\sim 15 \%$ ) of configurations. Finally, the figure shows the robustness of $n$-CPT sensitivity analysis: despite performing slightly worse than other strategies in some experiments, $n$-CPT sensitivity analysis never required to explore more than $5 \%$ of all configurations, even in problems with about $1 \%$ of optimal configurations.

\section{Discussion}

The results reported in Sect. 6 show that our proposed strategies can be employed to effectively revise at runtime the sanctions of the enforced norms to quickly improve the performance of the system (in terms of achievement of the system-level objectives). In particular, on 12 problems of different difficulty, our strategies reached optimal system's configurations after very few norm revisions. Starting with no initial knowledge about the effectiveness of the possible configurations, all the strategies explored on average less than $10 \%$ of all possible configurations before finding an optimal one. In the simplest experiment (DVN32), all strategies required to explore on average less than $1 \%$ of all possible configurations. In the same experiment, an uninformed strategy that does not consider runtime information and randomly tries a new configuration when the current one is not optimal 
would explore, on average, $62.5 \%$ of the configurations. In the most difficult experiment (DVN82), while a random strategy would explore on average $98.8 \%$ of the configurations to find one of the $1.2 \%$ optimal ones, our best performing strategy $n$-CPT sensitivity analysis explored, on average, only $5 \%$ of all possible configurations.

Our experiments identified three best-performing strategies: Naive synergy, State-based and $n$-CPT sensitivity analysis. We discuss each of these strategies and interpret the results and the conditions for their applicability.

Naive synergy determines, for each of the enforced norms, what type of synergy exists between the norm and the system-level objectives. Based on the identified synergy, Naive synergy increases or decreases the sanction for violating the norm. This strategy suits well cases where the observed data from MAS execution clearly highlights that a norm is better when either obeyed or violated. In experiment DUN81, for instance, in both contexts $\mathbf{c 2}$ and $\mathbf{c 3}$ the speed limit norm is effective only when fully obeyed by all agents (i.e., system configurations where some agents violate the speed limit are not optimal). In such experiment, and also in similar experiments such as DUN82 and DCN82, the results confirmed that Naive synergy outperforms the other strategies.

The State-based strategy extends Combined synergy. Just like the latter, it considers the synergy between norms and objectives. Unlike Combined synergy, it also considers the most likely explanation for the objectives being not achieved. Furthermore, Statebased takes also into account the global state of the system (the average norm obedience and objectives achievement) and suggests to revise only a certain type of norms at every iteration. This strategy is suitable for cases where many norms are enforced and where the obedience of agents to a norm is likely to affect also the obedience to other norms. In our experiments, State-based performed well in most of the cases, with the exception of the most difficult ones DCN81 and DVN82, where, similarly to Naive synergy it required a higher number of revisions.

Note that, in experiments $D C N 81$ and $D V N 82$, the optimal configurations are only 4 and 3, respectively, out of 256. To find the few optimal configurations quickly, it is necessary to have a strategy that precisely directs the norm revision. For this reason, synergy-based or category-based strategies, which only provide a direction for the revision (i.e., they simply suggest to either increase or decrease violations), were not the best in these experiments.

$n$-CPT sensitivity analysis, instead, provides a quantitative measure of how much change in the violations of each norm is required. This strategy is more precise, and, although it performed slightly worse than other strategies in a few cases, it showed a consistent convergence speed in all the experiments, including complex ones such as DCN81 and DVN82. Thus, this strategy proved to be the most robust in terms of convergence speed. It is worth noting, however, that in cases where the desired achievement of the system-level objectives is not particularly restrictive and where many norms are enforced, $n-C P T$ sensitivity analysis may be less adequate due to the higher computational effort it requires, especially if compared to simpler strategies like Naive synergy.

The worst-performing strategy, on average, is Naive sensitivity analysis. This strategy performed particularly bad (compared to the others) especially in the most difficult experiments, where, as explained above, very few configurations were needed to be found. This result, which may seem surprising since sensitivity-based strategies are generally more precise than the others, can be explained by the naive approach of the strategy in determining the amount of change in the violations of norms that is required to achieve the system-level objectives. In doing so, unlike $n$-CPT sensitivity analysis, 
this strategy considers the changes for only one norm at a time, assuming that the other parameters of the Bayesian Network (i.e., the amount of violations of other norms) would not change. After providing a suggestion, however, the strategy applies a sanctions revision to all norms together (i.e., it changes all the parameters of the network together), creating a discrepancy between the way the suggestions are provided and the implementation of such suggestions. This discrepancy appears evident in cases where the precision of the suggestions is essential to identify one of the few optimal solutions (e.g., $D C N 81$ and $D V N 82$ ). Note, however, that all the proposed strategies are heuristics. Therefore, there is no guarantee that one strategy will always perform better or worse than the others. This is visible in the results: every strategy that we proposed, including Naive sensitivity analysis, performed better than the others in at least one experiment.

\subsection{Limitations and possible extensions}

In the following, we provide a discussion of some of the limitations and assumptions related to our framework and to the revision strategies that we proposed, outlining some possible future directions.

\subsubsection{Preferences changing over time and context}

We considered agents with same preferences in all operating contexts. This simplification does not affect the generality of our approach. Our framework supports agents with different preferences in multiple operating contexts. In Sect. 4.2, we have shown how to use the estimation of the preferences of agents to determine an upper bound of the probability of violating a norm. In Sect. 5, we used such upper bound to guide the revision of the sanctions of the enforced norms in a certain operating context $c$. In order to use different preferences in varying operating contexts, it is possible to explicitly model the different contexts (as proposed, for example, in context-aware systems such as Ambient Intelligence systems [44]), and use an adequate upper bound in each of them.

This is made possible by the assumption that the preferences of agents (and therefore our estimation) do not change over time, i.e., we assumed that the behavior of the agent is consistent over time. We did not study the case of preferences changing over time. Preferences may change over time due to external factors inducing changes in the enduser's preferences, the introduction of new norms in the MAS, or changes in agents' own evaluation of states of affairs due to the acquisition of new experience $[40,58]$.

To support preferences that change over time, our framework needs to be adapted in a number of ways, briefly listed below. First, depending on the type of system, mechanisms for the dynamic elicitation of preferences should be employed and the estimation of the preferences should be dynamically replaced or updated (see, for example, mechanisms to learn and update dynamic preferences [19, 49]). Given the new preferences, the upper bound of the probability of violating a norm should be recomputed. System configurations that are ineffective when certain behaviours are exhibited by the agents, may be instead effective when different behaviors are exhibited, and vice-versa. When the preferences of the agents are changed, therefore, the knowledge acquired during the norm revision process about the effectiveness of the norms and about the relationship between norm violation and system-level objectives should be reconsidered and opportunely weighted. If the preferences of the agents change very quickly and repeatedly 
over time, the use of a static Norm Bayesian Network as the one described in Sect. 4 may be unfavourable and the use of different more dynamic learning techniques, e.g., Dynamic Bayesian Networks [42], may be necessary. Supporting partial and inaccurate preferences of agents, as briefly discussed in Sect. 7.1.2, could also help to cope with preferences changing over time.

\subsubsection{Partial or inaccurate information}

When looking for a new sanction set, we assumed not to have any knowledge about the norm violations that will be actually exhibited when a never-tried-before sanction set is used to enforce norms. To guide the norm revision, we used the upper bound of a norm violation, a "safe" estimation of the actual norm violation that will be exhibited by agents. To calculate such upper bound we assumed an accurate (i.e., perfect) estimation of the preferences of the agents concerning the aspects of the system we aim to regulate.

The advantage of having an accurate estimation of the preferences of the agents is that we can define an upper bound for the probability of violating a (well defined) norm that is not too coarse-grained (e.g., a trivial upper bound is obviously a probability of 1 , but this provides little information). As shown in Sect. 6, such an estimation, combined with our revision strategies, allows us to efficiently revise ineffective norms.

In some MASs, however, it is not possible to ensure a correct estimation of the agents' preferences [26]. Extending our work to support partial and/or inaccurate information about the agents' preferences requires an in-depth investigation. Based on the amount and type of information available, the accuracy and usefulness of the upper bound could significantly change. For partial information (e.g., we know that an agent type prefers a state of affairs over another, but we do not have information about all possible comparisons of alternative states of affairs), it is still possible to estimate a possibly more coarse-grained upper bound. For example, a trivial estimation could be obtained by assuming that agents always prefer to violate the norms related to aspects for which we do not have information. Less trivial estimations could be obtained for example by approximating the complete preferences by expressing the uncertain information as a belief function and leveraging the rationality principles of the preferences [17]. The estimated upper bound could be then refined over time by monitoring the behavior (i.e., the number of violations) of the agents. In case of inaccurate information (e.g., some of the available information about the preferences of agents is wrong, or the information available is only obtained from statistical data about the behavior of typical agents, or by learning the preferences from observed agents' choices [26]), the estimation of the probability of violating a norm should be treated more as a prediction, rather than an upper bound. In this case, techniques such as Bayesian Optimization [45], which attempts to find the minimum value of an unknown function, could be used for selecting new sanction sets and to refine over time the current estimation.

Nevertheless, a correct estimation of the preferences of the agents, as used in this paper, does not imply perfect revision strategies. This is because the trend of the upper bound may be different from the trend of the actual norm violation, which is unknown a priori. The consequence of this can be illustrated on the example of Fig. 10, which reports a comparison between an upper bound (red dashed line) of the probability of violating a norm $N$, and $N$ 's exhibited violation (blue solid line), w.r.t. the sanction associated to $N$. Suppose the current sanction for a norm $N$ is 0 , with an exhibited norm violation $P\left(N_{\text {viol }}\right)=0.3$, and the employed revision algorithm (e.g., Naive synergy) suggests to reduce violations of $N$. Here, the only possible choice for Algorithm 1, which relies on the estimation of the upper bound 


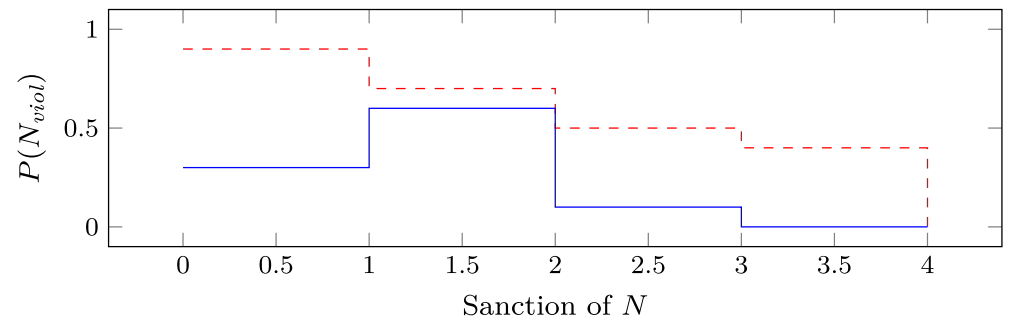

Fig. 10 Comparison between the upper bound (red dashed line) of the probability of violating a norm $N$, and $N$ 's exhibited violation (blue solid line), w.r.t. the sanction associated to $N$

of violating a norm, is to select sanction 4 as new sanction, since for all other sanctions the upper bound is higher than the currently exhibited norm violation. Although sanction 2 would also satisfy the suggestion, this will remain unknown until such sanction is tried. If the optimal value of $P\left(N_{\text {viol }}\right)$ for the achievement of the system-level objectives is, for instance, around 0.1 , our supervision framework will need to perform additional revision steps to select sanction 2 .

\subsubsection{Complexity of preferences representation}

In this paper, we introduced several types of preferences of rational agents as lists of tuples ordered according to different rational criteria. In our discussion and experiments, we considered complete preferences, i.e., we explicitly represented all possible alternative states of affairs. Such representation, however, grows exponentially with the number of norms and budgets. In real world scenarios, doing so may be possible only in restricted domains where the number of norms and the possible budgets of the agents is limited. In the general case, however, representing the complete preferences of agents may be infeasible. In this work we attempted to lay down well founded principles for understanding the interplay between norms and the preferences of rational agents. For this reason, we provided a formal definition of different types of rational agents and we studied the properties of their preferences in relation with the chances to violate the enforced norms. We consider this as a necessary starting point for approaches to the runtime supervision of normative multiagent systems involving rational agents. In Sect. 7.1.2, we outlined some guidelines for our framework to support also partial (and inaccurate) preferences, which is one obvious way to reduce the complexity of explicitly representing the complete preferences. We leave this as future work, together with the integration of automated preferences elicitation techniques within our framework.

\subsubsection{Norms importance}

Our strategies do not make any distinction between norms: revisions are applied to all the norms. This approach can be extended to support a selective revision that takes into account of the importance of a certain norm for the achievement of the objectives. Consider the derivative in Eq. (7), which describes the impact of changes in $P\left(N_{\text {viol }}\right)$ on $P\left(\mathbf{O}_{\text {true }}\right)$ in a context $\mathbf{c}$. High values of such derivatives imply that changes in the violations of norm $N$ have bigger impact on $P\left(\mathbf{O}_{\text {true }}\right)$. We call such derivative for a norm $N$ the importance 
[54] of norm $N$ in context c. By computing the importance of all norms, we obtain an ordering between norms w.r.t. the system-level objectives. The strategies of Sect. 5.1 could be then applied to the $k$ most important norms. Although there is no guarantee that this approach will be more effective, it applies to cases in which revising norms comes at a cost, and therefore minimizing the number of revisions is important.

In addition to the importance of a norm, the observed data from MAS execution allows to analyze the relationship between pairs of norms and to detect weather some of the following properties hold.

Additive synergy between two norms This property, based on the concept of additive synergies in qualitative probabilistic networks [55], describes a situation where it is more likely to achieve the objectives when two norms are either both obeyed or both violated. Formally, two norms $N 1$ and $N 2$ exhibit an additive synergy when $P\left(\mathbf{O}_{\text {true }} \mid N 1_{o b} N 2_{o b}\right)+P\left(\mathbf{O}_{\text {true }} \mid N 1_{\text {viol }} N 2_{\text {viol }}\right) \geqslant P\left(\mathbf{O}_{\text {true }} \mid N 1_{o b} N 2_{\text {viol }}\right)+P\left(\mathbf{O}_{\text {true }} \mid N 1_{\text {viol }} N 2_{o b}\right)$. The norms that exhibit an additive synergy with some of the $k$ most important ones, could also be considered among the norms to be revised.

Product synergy between two norms This property, based on the concept of product synergies in qualitative probabilistic networks [56], expresses how the value of one norm (e.g., $N 1$ obeyed) influences the probability of the values of another norm (e.g., $N 2$ obeyed), upon knowing the value for a common child (e.g., $\mathbf{O}$ true). For instance a negative product synergy says that observing $N 1$ obeyed makes less likely to observe $N 2$ being obeyed. Formally, two norms $N 1$ and $N 2$ exhibit a negative product synergy when $P\left(\mathbf{O}_{\text {true }} \mid N 1_{o b} N 2_{o b}\right) \cdot P\left(\mathbf{O}_{\text {true }} \mid N 1_{\text {viol }} N 2_{\text {viol }}\right) \geqslant(\leqslant) P\left(\mathbf{O}_{\text {true }} \mid N 1_{o b} N 2_{\text {viol }}\right) \cdot P\left(\mathbf{O}_{\text {true }} \mid N 1_{\text {viol }} N 2_{o b}\right)$. This property can be used to choose between two norms to revise: it is enough to revise one of them to obtain an effect on the other.

\subsubsection{Conflicting norms}

In this paper we assumed that the norms that are enforced are not conflicting, i.e., obeying a norm does not prevent a priori agents to obey other norms. This work focuses on regulative norms: norms enforced by an institution in order to regulate the behaviour of the agents so to achieve desired system-level properties. In this context, we believe that an institution should not enforce conflicting norms, and we rely on normative conflict resolution mechanisms [51]. Despite this, our framework currently supports conflicting norms as long as the agents are aware of such conflicts, i.e., as long as the preferences of agents already take into account the conflicts. If two norms $N_{1}$ and $N_{2}$ are conflicting, obeying $N_{1}$ prevents the agents to obey $N_{2}$ and vice-versa. The preference of an agent that is aware of the conflict, determines whether the agent prefers to obey $N_{1}$ and pay a sanction for $N_{2}$, or vice-versa. This information is sufficient in our framework to estimate the upper bound for the violation of the norms and revise the sanctions of the norms when needed. Additionally, the information of the conflict could also be explicitly used to improve the performance of our revision strategies, similarly to the use of the product synergies described in Sect. 7.1.4: if obeying a norm agents cannot obey another norm, then it is sufficient to revise one sanction to obtain an effect also on the violation of the other norm.

\subsubsection{Neighborhood expansion}

When a norm revision is triggered, our supervision mechanism searches for a new sanction set that satisfies the suggestions provided by one of the heuristic strategies. The neighborhood 
of a configuration, in the current hill climbing implementation of the supervisor, is composed by exactly one sanction set (configuration): the one that best satisfies the suggestions. An immediate extension of this approach is to expand the neighborhood definition, by including not only the best satisfying configuration, but also sub-optimal ones: those configurations that "almost" satisfy the suggestions provided. This extension is easily supported by our supervisor, and it better fits the typical usage of the hill climbing optimization technique. By expanding the neighborhood, the number of revision steps required by the supervision mechanism to find an optimal configuration could possibly further decrease. The challenge in expanding the neighborhood is in appropriately defining almost-satisfying suggestions. Different distance metrics and criteria could be considered in order to do so. Adopting a neighborhood composed only by the best satisfying configuration allowed us, however, to analyze the quality of the suggestions provided by our algorithms without further overloading the experimentation with additional parameters. Experiments with different neighborhood definitions will be carried on in future work, considering also a bigger case study.

\section{Conclusions}

In a MAS, the complexity and unpredictability of the agent interactions and of the environment must be taken into account to maximize the achievement of the system-level objectives. When engineering such systems, the available knowledge of these dynamics is only partial and incomplete. As a consequence, MASs need to be supervised and regulated at runtime.

In this paper, we proposed a supervision mechanism that relies on norms with sanction to influence agent behavior and regulate a MAS [11]. We considered MASs where agents are rational, i.e., they always choose to achieve their most preferred state of affairs. We characterized rational agents through their preferences and we made use of an estimation of the agents' preferences to guide the supervision of the MAS. Our mechanism automatically revises the sanctions that are employed to enforce the norms. To do so, it first interprets - through a Bayesian Network — observed data from MAS execution in terms of how well certain norms contribute to the achievement of the system-level objectives in different operating contexts. Then, it suggests how to revise the sanctions based on the knowledge learned at runtime and on the agents' preferences. We proposed six heuristics for the suggestion of sanction revisions.

An evaluation of the strategies through a traffic regulation simulation shows that our heuristics quickly identify optimal norm sets. We performed 12 different experiments on a ring-road traffic simulation, differing for the difficulty of the problem: the number of optimal norm sets to be found among all the possible ones ranged from $1.2 \%$ to $37.5 \%$. All the proposed strategies explored a small number of norm sets before finding an optimal one. In particular, the strategy $n$-CPT sensitivity analysis, based on the sensitivity analysis technique from probabilistic reasoning [14], on average never required to explore more than 5\% of all possible norm sets in order to find one of the optimal ones.

This work paves the way for numerous future directions, some of which are sketched in Sect. 7.1. An in-depth evaluation of the scalability and computational complexity of the presented approach is necessary to assess its suitability for MASs with many norms and sanctions. Our simple language for representing norms and agents' preferences can be extended to consider complex norm types beyond atomic propositions. Our agent population was defined according to specific types. Future work should study the effect of agents 
that deviate from the prototypical agent types. Finally, we are planning to extend our strategies to support, in addition to the revision of the sanctions, also the revision of the norm proposition, and to synthesize new norms.

Acknowledgements We thank Dr. Silja Renooij for her advice and support on the issues related to sensitivity analysis.

Open Access This article is licensed under a Creative Commons Attribution 4.0 International License, which permits use, sharing, adaptation, distribution and reproduction in any medium or format, as long as you give appropriate credit to the original author(s) and the source, provide a link to the Creative Commons licence, and indicate if changes were made. The images or other third party material in this article are included in the article's Creative Commons licence, unless indicated otherwise in a credit line to the material. If material is not included in the article's Creative Commons licence and your intended use is not permitted by statutory regulation or exceeds the permitted use, you will need to obtain permission directly from the copyright holder. To view a copy of this licence, visit http://creativecommons.org/licenses/by/4.0/.

\section{Appendix 1: Properties of rational agents' preferences}

We report here a formal definition of the properties of the rational agents' preferences described in Sects. 3.3 and 4.2.

Proposition 1 A basic preference Pref $(a)=(A, \succeq)$ for an agent $a \in A g$ is

- transitive $\forall x, y, z \in A$ if $x \geq y$ and $y \geq z$ then $x \geq z$; and

- complete $\forall x, y \in A$ either $x \geq y$ or $y \geq x$ or $x \sim y$.

Proof Consider a list $A L=\left(L_{1}, \ldots, L_{n}\right)$, a set $\mathcal{B} \subset \mathbb{N}$, a set $B L \subseteq \mathcal{B}^{n}$ and an ordered set $\mathcal{N}$ of $n$ norms. Let $\operatorname{Pref}(a)=(A, \geq)$ be a basic preference and $x, y, z$ be alternatives in $A$.

(Transitivity) Assume that $x \geq y$ and $y \geq z$. We prove the transitivity for two cases: either the preference adheres to Definition 2a (case 1) or the preference adheres to Definition $2 \mathrm{~b}$ (case 2). For both cases, we show that $x \geq z$.

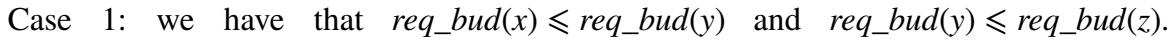

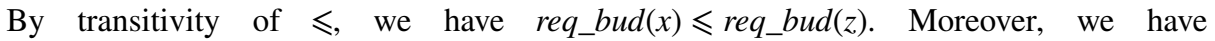
$\forall k, l \in A, \forall B, B^{\prime} \in B L: k[B]>l[B] \Rightarrow k\left[B^{\prime}\right]>l\left[B^{\prime}\right]$ for both $x \geq y$ and $y \geq z$, such that we have it also for $x \geq z$. Therefore, we conclude that $x \geq z$.

Case 2: we have either (i) $\operatorname{prop}(x)=\operatorname{prop}(y)=\operatorname{prop}(z)$ or (ii) $\operatorname{prop}(x) \neq \operatorname{prop}(y)$ and either $\operatorname{prop}(y)=\operatorname{prop}(z)$ or $\operatorname{prop}(y) \neq \operatorname{prop}(z)$. In case (i), we have that req_bud $(x) \leqslant r e q \_b u d(y)$ and $r e q \_b u d(y) \leqslant r e q \_b u d(z)$, and, by transitivity of $\leqslant$, we have that the first condition of Definition $2 \mathrm{~b}$ is also satisfied by $x$ and $z$. In case (ii), instead, we have that $\forall B, B^{\prime} \in B L: x[B] \geq y\left[B^{\prime}\right]$. If $\operatorname{prop}(y)=\operatorname{prop}(z)$, we have that $\operatorname{prop}(x) \neq \operatorname{prop}(z)$ and $\forall B, B^{\prime} \in B L: x[B] \geq z\left[B^{\prime}\right]$, so the second condition of Definition $2 \mathrm{~b}$ is satisfied also by $x$ and $z$. If instead also $\operatorname{prop}(y) \neq \operatorname{prop}(z)$, then we have that $\forall B, B^{\prime} \in B L: x[B] \geq y\left[B^{\prime}\right]$ and $\forall B, B^{\prime} \in B L: y[B] \geq z\left[B^{\prime}\right]$. Take three arbitrary alternatives $x[B], y\left[B^{\prime}\right], z\left[B^{\prime \prime}\right]$. We have that $x[B] \geq y\left[B^{\prime}\right]$ and $y\left[B^{\prime}\right] \geq z\left[B^{\prime \prime}\right]$. Since the choice of $B, B^{\prime}$ and $B^{\prime \prime}$ was arbitrary, this holds for any possible budget list. Therefore there is no $B, B^{\prime \prime} \in B L$ such that alternative $z\left[B^{\prime \prime}\right]$ is strictly preferred to an alternative $x[B]$, i.e., $\forall B, B^{\prime \prime} \in B L: x[B] \geq z\left[B^{\prime \prime}\right]$. Again the second condition of Definition $2 \mathrm{~b}$ is satisfied by $x$ and $z$, such that $x \geq z$.

(Completeness) By definition of basic preference, every pair of alternatives $x, y \in A$ has to satisfy either Definition 2a or Definition 2b. Notice that, given $x$ and $y$, if it is not the 
case that $x \geq y$, then we have that $y>x$, therefore for every pair of alternatives $x, y \in A$ either $x \geq y$ or $y>x$.

\section{Proposition 2 A preference Pref $(a)=(A, \geq)$ for an agent $a \in A g$ is}

- transitive $\forall x, y, z \in A$ if $x \geq y$ and $y \geq z$ then $x \geq z$; and

- complete $\forall x, y \in A$ either $x \geq y$ or $y \geq x$ or $x \sim y$.

Proof Consider a list $A L=\left(L_{1}, \ldots, L_{n}\right)$, a set $\mathcal{B} \subset \mathbb{N}$, a set $B L \subseteq \mathcal{B}^{n}$ and an ordered set $\mathcal{N}$ of $n$ norms. Let $A=\left\{\left(\left\langle p_{1}, b_{1}\right\rangle, \ldots,\left\langle p_{n}, b_{n}\right\rangle\right) \mid p_{i} \in L_{i} \&\left(b_{1}, \ldots, b_{n}\right) \in B L\right\}$ be the set of alternatives over which agents have preferences. Let $A_{1}, \ldots, A_{k}$ be $k$ disjoint subsets of $A$ as per Definition 4, and $x, y, z$ be alternatives in $A$.

(Transitivity) Assume that $x \geq y$ and $y \geq z$. If both $x, y$ and $z$ belong to the same $A_{i}$ for $1 \leqslant i \leqslant k$ then, by Proposition $1, x \geq z$. Otherwise, if $x \in A_{i}, y \in A_{j}$ and $z \in A_{l}$ with $i<j<l$, then, by Definition 4, given $i<l, \forall v \in A_{i} \forall w \in A_{l}: v \geq w$, therefore $x \geq z$.

(Completeness) By Proposition 1, for every pair of alternatives $x, y \in A_{i}$ for $1 \leqslant i \leqslant k$, either $x \succeq_{i} y$ or $y \succeq_{i} x$. Furthermore, by definition of preference, for all $x \in A_{j}$ and $y \in A_{i}$, we have $x \geq y$, for $1 \leqslant j<i \leqslant k$. We have therefore that for every pair of alternatives $x, y \in A$ either $x \geq y$ or $y \geq x$.

Proposition 3 Given an ordered set of norms $\mathcal{N}=\left\langle N_{1}, \ldots, N_{n}\right\rangle$, and a set of t agent types $\mathcal{T}$, each type corresponding to a consistent preference (as per Definition 5), increasing the sanction of a norm $N_{j}$ in $\mathcal{N}$ without changing the sanctions of other norms, does not increase the upper bound of the probability $P\left(N_{\text {viol }}\right)$, i.e., $U B\left(N_{\text {viol }}, \mathcal{N}\right)$, for all $N$ in $\mathcal{N}$.

Proof In this paper the agent's preferences are not affected by the preferences of other agents. Since the upper bound $U B\left(N_{\text {viol }}, \mathcal{N}\right)$ of the probability of violating a norm $N$ in the context of a norm set $\mathcal{N}$ is determined by the number of agents with reason to violate $N$, as per Sect. 3.2, if Proposition 3 holds for one agent type, then Proposition 3 must hold also for all agent types. In the following we consider, therefore, one agent type $T$. Furthermore we assume $\mathcal{N}$ composed by at least two different norms (if only one norm is enforced, Proposition 3 is trivially satisfied).

We prove Proposition 3 by contradiction.

Let $M$ be the set of most preferred alternatives to act upon for agent type $T$ in the context of $\mathcal{N}=\left\langle N_{1}, \ldots, N_{j}, \ldots, N_{n}\right\rangle$ (as per Definition 6). Suppose we increase the sanction of norm $N_{j}=\left(p_{j}, s_{j}\right)$, obtaining $N_{j}^{\prime}=\left(p_{j}, s_{j}^{\prime}>s_{j}\right)$. Let now $M^{\prime}$ be the set of most preferred alternatives to act upon for agent type $T$ in the context of $\mathcal{N}^{\prime}=\left\langle N_{1}, \ldots, N_{j}^{\prime}, \ldots, N_{n}\right\rangle$.

Suppose, by contradiction, that $U B\left(N_{\text {viol }}, \mathcal{N}^{\top}\right)>U B\left(N_{\text {viol }}, \mathcal{N}\right)$ for $N=(p, s) \neq N_{j}$, with $p \in L_{i}$ and $L_{i}$ in $A L$. This means that, in the context of $\mathcal{N}, T$ has no reason to violate $N$, while in the context of $\mathcal{N}, T$ has reason to violate $N$ (i.e., there exists no alternative $c \in M$ with $\operatorname{viol}(c, N)$, while there exists an alternative $c^{\prime} \in M^{\prime}$ such that $\left.\operatorname{viol}\left(c^{\prime}, N\right)\right)$.

In order to modify the set of most preferred alternatives $M$ when increasing the sanction from $s_{j}$ to $s_{j}^{\prime}$, it must be the case that there exists at least one alternative $c \in M$ s.t. $\operatorname{viol}\left(c, N_{j}\right)$ and $s_{j} \leqslant b_{j}<s_{j}^{\prime}$ (with $b_{j}$ budget of the $j$-th pair in $c$ ). If it is not the case increasing $s_{j}$ to $s_{j}^{\prime}$ does not affect $T$ 's most preferred alternatives and thus the proposition holds.

Consider the alternative from $M$ with highest budget $b_{j}$ in the $j$-th pair. Consider also an $i \neq j$. Let $\bar{a} w x[B]=\left(\left\langle p_{1}, b_{1}\right\rangle, \ldots,\left\langle w, b_{w}\right\rangle, \ldots,\left\langle x, b_{x}\right\rangle, \ldots,\left\langle p_{n}, b_{n}\right\rangle\right)$ be such alternative, with 
$w \in L_{j}, x \in L_{i}$ and $s_{j} \leqslant b_{w}<s_{j}^{\prime}$. Let $\bar{b} z y\left[B^{\prime}\right]=\left(\left\langle p_{1}^{\prime}, b_{1}^{\prime}\right\rangle, \ldots,\left\langle z, b_{z}\right\rangle, \ldots,\left\langle y, b_{y}\right\rangle, \ldots,\left\langle p_{n}^{\prime}, b_{n}^{\prime}\right\rangle\right)$ be an alternative $c^{\prime} \in M^{\prime}$ such that $c^{\prime} \notin M$ and $\operatorname{viol}\left(c^{\prime}, N\right)$ with $z \in L_{j}, y \in L_{i}$ and $b_{z} \geqslant s_{j}^{\prime}$. Notice that $\bar{a} w x[B]$ is compliant w.r.t. $N$ and $\bar{b} z y\left[B^{\prime}\right]$ is not compliant w.r.t. $N$, hence $y \neq x$. Notice also that $\bar{a} w x[B]>\bar{b} z y\left[B^{\prime}\right]$. This is because by Definition 6 we have that, since $\bar{b} z y\left[B^{\prime}\right] \notin M, \bar{b} z y\left[B^{\prime}\right] \geq \bar{a} w x[B]$ iff $\bar{b} z y\left[B^{\prime}\right]$ violates a norm $N_{k}$ but the budget is not enough to pay the sanction. Such $N_{k}$ cannot be $N_{j}$, since $b_{z} \geqslant s_{j}^{\prime}$, and if it's another $N_{k}$ then $\underline{b} z y\left[B^{\prime}\right]$ cannot be also in $M^{\prime}$ because we only increased the sanction of norm $N_{j}$. Therefore $\bar{b} z y\left[B^{\prime}\right]$ must be strictly less preferred than $\bar{a} w x[B]$. Furthermore, let $\bar{c}$ be a fully compliant alternative. $^{10}$

We first consider the case of $T=(A, \geq)$ basic preference as per Definition 2, which can adhere to either Definition 2a or Definition 2b, then we uplift the proof to the preference as per Definition 4.

\section{Basic preference}

(Case Definition 2a) Since $\bar{a} w x[B]>\bar{b} z y\left[B^{\prime}\right]$, it holds that, due to Definition 2a, $c[B]>\bar{b} z y\left[B^{\prime}\right]$ for all alternatives $c$ in $A$. This means that also a fully compliant alternative $\bar{c}[B]$ is such that $\bar{c}[B]>\bar{b} z y\left[B^{\prime}\right]$. However, since $s_{j}^{\prime}>b_{w}$, after revising $s_{j}$ into $s_{j}^{\prime}$, there is at least one alternative in $M^{\prime}$ (i.e., $\bar{c}[B]$ ) that is strictly preferred to $\bar{b} z y\left[B^{\prime}\right]$, because already present in $M$, and that is compliant to $N$. Therefore, $\bar{b} z y\left[B^{\prime}\right]$ cannot be among the most preferred alternatives to act upon, i.e., $\bar{b} z y\left[B^{\prime}\right] \notin M^{\prime}$ (contradiction).

(Case Definition 2b) Since $\bar{a} w x[B] \in M>\bar{b} z y\left[B^{\prime}\right] \in M^{\prime}$, by Definition $2 \mathrm{~b}$ it holds that $\bar{a} w x\left[B^{\prime}\right] \geq \bar{a} w x[B]>\bar{b} z y\left[B^{\prime}\right] \geq \bar{b} z y[B]$ for all $B^{\prime} \in B L$, i.e., $\bar{a} w x>\bar{b} z y$ regardless of the required budget, including the maximum possible budget in $\mathcal{B}, \max (\mathcal{B})$. Therefore, since $\bar{a} w x$ is the alternative in $M$ with highest budget in the $j$-th pair, we have that $s_{j}^{\prime}>\max (\mathcal{B})$, and no alternative that violates $N_{j}^{\prime}$ can be chosen in the context of $\mathcal{N} . \bar{b} z y\left[B^{\prime}\right]$ is then compliant w.r.t. $N_{j}^{\prime}$, hence $z \neq w$. By Definition 2, $T$ contains at least another alternative $\bar{a} w y\left[B^{\prime}\right]$. If $\bar{a} w y\left[B^{\prime}\right] \geq \bar{a} w x\left[B^{\prime}\right]$, then, according to Definition $2 \mathrm{~b}, \bar{a} w y\left[B^{\prime}\right] \geq \bar{a} w x\left[B^{\prime}\right]$ for all $B^{\prime} \in B L$. But if this is the case, we have that $s_{j}>\max (\mathcal{B})$ (otherwise at least one alternative $\bar{a} w y$ with $b_{w}=\max (\mathcal{B})$ is in $M$ and, in contradiction with out hypothesis, $T$ has reason to violate $N$ since $\operatorname{viol}(y, N))$. But if $s_{j}>\max (\mathcal{B})$ we have $\bar{a} w x[B] \notin M$ (contradiction). If, instead, $\bar{a} w x\left[B^{\prime}\right]>\bar{a} w y\left[B^{\prime}\right]$ then $\bar{a} w x>\bar{a} w y$ regardless of the budget. By consistency (Definition 5), then, we also have $\bar{b} z x>\bar{b} z y$. We distinguish 2 cases: (a) $\bar{a} w x>\bar{b} z x$, this implies $\bar{a} w x>\bar{b} z x>\bar{b} z y$, which contradicts $\bar{b} z y\left[B^{\prime}\right] \in M^{\prime}$, since alternatives $\bar{b} z x$ (compliant w.r.t $N_{j}^{\prime}$ ) are strictly preferred to $\bar{b} z y\left[B^{\prime}\right]$; (b) $\bar{b} z x>\bar{a} w x$, this implies that, since $\bar{a} w x[B] \in M$ and $\bar{b} z x$ is compliant w.r.t. both $L_{j}$ and $L_{i}$, then for every other norm violated by $\bar{b} z x$, the sanction associated to such norm is bigger than $\max (\mathcal{B})$ (otherwise $\bar{a} w x \notin M$ and at least one alternative $\bar{b} z x \in M)$. But if this is the case, also $\bar{b} z y\left[B^{\prime}\right] \notin M^{\prime}$, since the only sanction that we change is $s_{j}$, and again we have a contradiction.

\section{Preference}

In the case of preference $T=(A, \succeq)$, the $k$ basic preferences composing $A$ adhere to either Definition $2 \mathrm{a}$ or Definition $2 \mathrm{~b}$. The only case non considered above is when $\bar{a} w x[B] \in A_{i}, \bar{b} z y\left[B^{\prime}\right] \in A_{p}$ and it does not exists an alternative $c \in A_{p}$ s.t. $\bar{a} w x[B]>c$, and it does not exists an alternative $c^{\prime} \in A_{q}$ s.t. $c^{\prime}>\bar{b} z y\left[B^{\prime}\right]$, for two basic preferences $A_{p}, A_{q}$ composing $A$, with $1 \leqslant p<q \leqslant k$. Since all alternatives in $A_{i}$ have required budget lower or equal than $\max \left(\mathcal{B}_{i}\right)$, then, due to Definition 2 , among $A_{p}$ there is at least one fully compliant alternative with required budget $\leqslant \max \left(\mathcal{B}_{i}\right)$. Therefore, even if sanction for $N_{j}$ is increased

\footnotetext{
${ }_{10}$ We call $\bar{a}$ and $\bar{b}$ the list of propositional atoms that are different from $w, x, y, z$ respectively in $\bar{a} w x$ and $\bar{b} z y$. Also, we use notation $c[B]$ to indicate an alternative $c$ with list of budgets $B$, as described in Sect. 3.2.
} 
to a value $s>\max (\mathcal{B}), M^{\prime}$ does not contain any alternative from $A_{q}$ that was not already in $M$, therefore $\bar{b} z y\left[B^{\prime}\right] \notin M^{\prime}$, and again we have a contradiction.

\section{Appendix 2: Experiments agent types}

We report here the full preferences of the four types of agents considered in our experimentation, described in Sect. 6.

\section{- BraveRich}

$$
\begin{aligned}
& \left.\left(\left\langle s p_{15}, 0\right\rangle,\left\langle\text { dist }_{0.5}, 0\right\rangle\right)\right\rangle\left(\left\langle s p_{15}, 0\right\rangle,\left\langle\text { dist }_{0.5}, 1\right\rangle\right) \geq\left(\left\langle s p_{15}, 1\right\rangle,\left\langle\text { dist }_{0.5}, 0\right\rangle\right) \\
& >\left(\left\langle s p_{15}, 1\right\rangle,\left\langle\text { dist }_{0.5}, 1\right\rangle\right) \geq\left(\left\langle s p_{15}, 0\right\rangle,\left\langle\text { dist }_{0.5}, 2\right\rangle\right) \geq\left(\left\langle s p_{15}, 2\right\rangle,\left\langle\text { dist }_{0.5}, 0\right\rangle\right) \\
& >\left(\left\langle s p_{15}, 1\right\rangle,\left\langle\text { dist }_{0.5}, 2\right\rangle\right) \geq\left(\left\langle s p_{15}, 2\right\rangle,\left\langle\text { dist }_{0.5}, 1\right\rangle\right) \\
& >\left(\left\langle s p_{15}, 2\right\rangle,\left\langle\text { dist }_{0.5}, 2\right\rangle\right) \\
& >\left(\left\langle s p_{8}, 0\right\rangle,\left\langle\text { dist }_{0.5}, 0\right\rangle\right)>\left(\left\langle s p_{8}, 0\right\rangle,\left\langle\text { dist }_{0.5}, 1\right\rangle\right) \geq\left(\left\langle s p_{8}, 1\right\rangle,\left\langle\text { dist }_{0.5}, 0\right\rangle\right) \\
& >\left(\left\langle s p_{8}, 1\right\rangle,\left\langle\text { dist }_{0.5}, 1\right\rangle\right) \geq\left(\left\langle s p_{8}, 0\right\rangle,\left\langle\text { dist }_{0.5}, 2\right\rangle\right) \geq\left(\left\langle s p_{8}, 2\right\rangle,\left\langle\text { dist }_{0.5}, 0\right\rangle\right) \\
& \left.>\left(\left\langle s p_{8}, 1\right\rangle,\left\langle\text { dist }_{0.5}, 2\right\rangle\right) \geq\left(\left\langle s p_{8}, 2\right\rangle,\left\langle\text { dist }_{0.5}, 1\right\rangle\right)\right\rangle\left(\left\langle s p_{8}, 2\right\rangle,\left\langle\text { dist }_{0.5}, 2\right\rangle\right) \\
& \left.>\left(\left\langle s p_{3}, 0\right\rangle,\left\langle\text { dist }_{0.5}, 0\right\rangle\right)\right\rangle\left(\left\langle s p_{3}, 0\right\rangle,\left\langle\text { dist }_{0.5}, 1\right\rangle\right) \geq\left(\left\langle s p_{3}, 1\right\rangle,\left\langle\text { dist }_{0.5}, 0\right\rangle\right) \\
& >\left(\left\langle s p_{3}, 1\right\rangle,\left\langle\text { dist }_{0.5}, 1\right\rangle\right) \geq\left(\left\langle s p_{3}, 0\right\rangle,\left\langle\text { dist }_{0.5}, 2\right\rangle\right) \geq\left(\left\langle s p_{3}, 2\right\rangle,\left\langle\text { dist }_{0.5}, 0\right\rangle\right) \\
& >\left(\left\langle s p_{3}, 1\right\rangle,\left\langle\text { dist }_{0.5}, 2\right\rangle\right) \geq\left(\left\langle s p_{3}, 2\right\rangle,\left\langle\text { dist }_{0.5}, 1\right\rangle\right) \\
& \left.\left.>\left(\left\langle s p_{3}, 2\right\rangle,\left\langle\text { dist }_{0.5}, 2\right\rangle\right)\right\rangle\left(\left\langle s p_{15}, 0\right\rangle,\left\langle\text { dist }_{1}, 0\right\rangle\right)\right\rangle\left(\left\langle s p_{15}, 0\right\rangle,\left\langle\text { dist }_{1}, 1\right\rangle\right) \\
& \left.\geq\left(\left\langle s p_{15}, 1\right\rangle,\left\langle\text { dist }_{1}, 0\right\rangle\right)\right\rangle\left(\left\langle s p_{15}, 1\right\rangle,\left\langle\text { dist }_{1}, 1\right\rangle\right) \geq\left(\left\langle s p_{15}, 0\right\rangle,\left\langle\text { dist }_{1}, 2\right\rangle\right) \\
& \left.\geq\left(\left\langle s p_{15}, 2\right\rangle,\left\langle\text { dist }_{1}, 0\right\rangle\right)\right\rangle\left(\left\langle s p_{15}, 1\right\rangle,\left\langle\text { dist }_{1}, 2\right\rangle\right) \geq\left(\left\langle s p_{15}, 2\right\rangle,\left\langle\text { dist }_{1}, 1\right\rangle\right) \\
& \left.\left.>\left(\left\langle s p_{15}, 2\right\rangle,\left\langle\text { dist }_{1}, 2\right\rangle\right)\right\rangle\left(\left\langle s p_{8}, 0\right\rangle,\left\langle\text { dist }_{1}, 0\right\rangle\right)\right\rangle\left(\left\langle s p_{8}, 0\right\rangle,\left\langle\text { dist }_{1}, 1\right\rangle\right) \\
& \left.\geq\left(\left\langle s p_{8}, 1\right\rangle,\left\langle\text { dist }_{1}, 0\right\rangle\right)\right\rangle\left(\left\langle s p_{8}, 1\right\rangle,\left\langle\text { dist }_{1}, 1\right\rangle\right) \geq\left(\left\langle s p_{8}, 0\right\rangle,\left\langle\text { dist }_{1}, 2\right\rangle\right) \\
& \left.\geq\left(\left\langle s p_{8}, 2\right\rangle,\left\langle\text { dist }_{1}, 0\right\rangle\right)\right\rangle\left(\left\langle s p_{8}, 1\right\rangle,\left\langle\text { dist }_{1}, 2\right\rangle\right) \geq\left(\left\langle s p_{8}, 2\right\rangle,\left\langle\text { dist }_{1}, 1\right\rangle\right) \\
& \left.\left.>\left(\left\langle s p_{8}, 2\right\rangle,\left\langle\text { dist }_{1}, 2\right\rangle\right)\right\rangle\left(\left\langle s p_{3}, 0\right\rangle,\left\langle\text { dist }_{1}, 0\right\rangle\right)\right\rangle\left(\left\langle s p_{3}, 0\right\rangle,\left\langle\text { dist }_{1}, 1\right\rangle\right) \\
& \left.\geq\left(\left\langle s p_{3}, 1\right\rangle,\left\langle\text { dist }_{1}, 0\right\rangle\right)\right\rangle\left(\left\langle s p_{3}, 1\right\rangle,\left\langle\text { dist }_{1}, 1\right\rangle\right) \geq\left(\left\langle s p_{3}, 0\right\rangle,\left\langle\text { dist }_{1}, 2\right\rangle\right) \\
& \left.\geq\left(\left\langle s p_{3}, 2\right\rangle,\left\langle\text { dist }_{1}, 0\right\rangle\right)\right\rangle\left(\left\langle s p_{3}, 1\right\rangle,\left\langle\text { dist }_{1}, 2\right\rangle\right) \geq\left(\left\langle s p_{3}, 2\right\rangle,\left\langle\text { dist }_{1}, 1\right\rangle\right) \\
& \left.\left.>\left(\left\langle s p_{3}, 2\right\rangle,\left\langle\text { dist }_{1}, 2\right\rangle\right)\right\rangle\left(\left\langle s p_{15}, 0\right\rangle,\left\langle\text { dist }_{2}, 0\right\rangle\right)\right\rangle\left(\left\langle s p_{15}, 0\right\rangle,\left\langle\text { dist }_{2}, 1\right\rangle\right) \\
& \left.\geq\left(\left\langle s p_{15}, 1\right\rangle,\left\langle\text { dist }_{2}, 0\right\rangle\right)\right\rangle\left(\left\langle s p_{15}, 1\right\rangle,\left\langle\text { dist }_{2}, 1\right\rangle\right) \geq\left(\left\langle s p_{15}, 0\right\rangle,\left\langle\text { dist }_{2}, 2\right\rangle\right) \\
& \left.\geq\left(\left\langle s p_{15}, 2\right\rangle,\left\langle\text { dist }_{2}, 0\right\rangle\right)\right\rangle\left(\left\langle s p_{15}, 1\right\rangle,\left\langle\text { dist }_{2}, 2\right\rangle\right) \geq\left(\left\langle s p_{15}, 2\right\rangle,\left\langle\text { dist }_{2}, 1\right\rangle\right) \\
& \left.\left.>\left(\left\langle s p_{15}, 2\right\rangle,\left\langle\text { dist }_{2}, 2\right\rangle\right)\right\rangle\left(\left\langle s p_{8}, 0\right\rangle,\left\langle\text { dist }_{2}, 0\right\rangle\right)\right\rangle\left(\left\langle s p_{8}, 0\right\rangle,\left\langle\text { dist }_{2}, 1\right\rangle\right) \\
& \left.\geq\left(\left\langle s p_{8}, 1\right\rangle,\left\langle\text { dist }_{2}, 0\right\rangle\right)\right\rangle\left(\left\langle s p_{8}, 1\right\rangle,\left\langle\text { dist }_{2}, 1\right\rangle\right) \geq\left(\left\langle s p_{8}, 0\right\rangle,\left\langle\text { dist }_{2}, 2\right\rangle\right) \\
& \left.\geq\left(\left\langle s p_{8}, 2\right\rangle,\left\langle\text { dist }_{2}, 0\right\rangle\right)\right\rangle\left(\left\langle s p_{8}, 1\right\rangle,\left\langle\text { dist }_{2}, 2\right\rangle\right) \geq\left(\left\langle s p_{8}, 2\right\rangle,\left\langle\text { dist }_{2}, 1\right\rangle\right) \\
& \left.\left.>\left(\left\langle s p_{8}, 2\right\rangle,\left\langle\text { dist }_{2}, 2\right\rangle\right)\right\rangle\left(\left\langle s p_{3}, 0\right\rangle,\left\langle\text { dist }_{2}, 0\right\rangle\right)\right\rangle\left(\left\langle s p_{3}, 0\right\rangle,\left\langle\text { dist }_{2}, 1\right\rangle\right) \\
& \left.\geq\left(\left\langle s p_{3}, 1\right\rangle,\left\langle\text { dist }_{2}, 0\right\rangle\right)\right\rangle\left(\left\langle s p_{3}, 1\right\rangle,\left\langle\text { dist }_{2}, 1\right\rangle\right) \geq\left(\left\langle s p_{3}, 0\right\rangle,\left\langle\text { dist }_{2}, 2\right\rangle\right) \\
& \left.\geq\left(\left\langle s p_{3}, 2\right\rangle,\left\langle\text { dist }_{2}, 0\right\rangle\right)\right\rangle\left(\left\langle s p_{3}, 1\right\rangle,\left\langle\text { dist }_{2}, 2\right\rangle\right) \geq\left(\left\langle s p_{3}, 2\right\rangle,\left\langle\text { dist }_{2}, 1\right\rangle\right) \\
& >\left(\left\langle s p_{3}, 2\right\rangle,\left\langle\text { dist }_{2}, 2\right\rangle\right)
\end{aligned}
$$


- BraveMiddleClass

$$
\begin{aligned}
& \left(\left\langle s p_{15}, 0\right\rangle,\left\langle\text { dist }_{0.5}, 0\right\rangle\right)>\left(\left\langle s p_{15}, 0\right\rangle,\left\langle\text { dist }_{0.5}, 1\right\rangle\right) \geq\left(\left\langle s p_{15}, 1\right\rangle,\left\langle\text { dist }_{0.5}, 0\right\rangle\right) \\
& \quad>\left(\left\langle s p_{15}, 1\right\rangle,\left\langle\text { dist }_{0.5}, 1\right\rangle\right)>\left(\left\langle s p_{8}, 0\right\rangle,\left\langle\text { dist }_{0.5}, 0\right\rangle\right)>\left(\left\langle s p_{8}, 0\right\rangle,\left\langle\text { dist }_{0.5}, 1\right\rangle\right) \\
& \quad \geq\left(\left\langle s p_{8}, 1\right\rangle,\left\langle\text { dist }_{0.5}, 0\right\rangle\right)>\left(\left\langle s p_{8}, 1\right\rangle,\left\langle\text { dist }_{0.5}, 1\right\rangle\right)>\left(\left\langle s p_{3}, 0\right\rangle,\left\langle\text { dist }_{0.5}, 0\right\rangle\right) \\
& \quad>\left(\left\langle s p_{3}, 0\right\rangle,\left\langle\text { dist }_{0.5}, 1\right\rangle\right) \geq\left(\left\langle s p_{3}, 1\right\rangle,\left\langle\text { dist }_{0.5}, 0\right\rangle\right)>\left(\left\langle s p_{3}, 1\right\rangle,\left\langle\text { dist }_{0.5}, 1\right\rangle\right) \\
& \quad>\left(\left\langle s p_{15}, 0\right\rangle,\left\langle\text { dist }_{1}, 0\right\rangle\right)>\left(\left\langle s p_{15}, 0\right\rangle,\left\langle\text { dist }_{1}, 1\right\rangle\right) \geq\left(\left\langle s p_{15}, 1\right\rangle,\left\langle\text { dist }_{1}, 0\right\rangle\right) \\
& \quad>\left(\left\langle s p_{15}, 1\right\rangle,\left\langle\text { dist }_{1}, 1\right\rangle\right)>\left(\left\langle s p_{8}, 0\right\rangle,\left\langle\text { dist }_{1}, 0\right\rangle\right)>\left(\left\langle s p_{8}, 0\right\rangle,\left\langle\text { dist }_{1}, 1\right\rangle\right) \\
& \geq\left(\left\langle s p_{8}, 1\right\rangle,\left\langle\text { dist }_{1}, 0\right\rangle\right)>\left(\left\langle s p_{8}, 1\right\rangle,\left\langle\text { dist }_{1}, 1\right\rangle\right)>\left(\left\langle s p_{3}, 0\right\rangle,\left\langle\text { dist }_{1}, 0\right\rangle\right) \\
& \quad>\left(\left\langle s p_{3}, 0\right\rangle,\left\langle\text { dist }_{1}, 1\right\rangle\right) \geq\left(\left\langle s p_{3}, 1\right\rangle,\left\langle\text { dist }_{1}, 0\right\rangle\right)>\left(\left\langle s p_{3}, 1\right\rangle,\left\langle\text { dist }_{1}, 1\right\rangle\right) \\
& \quad>\left(\left\langle s p_{15}, 0\right\rangle,\left\langle\text { dist }_{2}, 0\right\rangle\right)>\left(\left\langle s p_{15}, 0\right\rangle,\left\langle\text { dist }_{2}, 1\right\rangle\right) \geq\left(\left\langle s p_{15}, 1\right\rangle,\left\langle\text { dist }_{2}, 0\right\rangle\right) \\
& \quad>\left(\left\langle s p_{15}, 1\right\rangle,\left\langle\text { dist }_{2}, 1\right\rangle\right)>\left(\left\langle s p_{8}, 0\right\rangle,\left\langle\text { dist }_{2}, 0\right\rangle\right)>\left(\left\langle s p_{8}, 0\right\rangle,\left\langle\text { dist }_{2}, 1\right\rangle\right) \\
& \quad \geq\left(\left\langle s p_{8}, 1\right\rangle,\left\langle\text { dist }_{2}, 0\right\rangle\right)>\left(\left\langle s p_{8}, 1\right\rangle,\left\langle\text { dist }_{2}, 1\right\rangle\right)>\left(\left\langle s p_{3}, 0\right\rangle,\left\langle\text { dist }_{2}, 0\right\rangle\right) \\
& \left.\quad>\left(\left\langle s p_{3}, 0\right\rangle,\left\langle\text { dist }_{2}, 1\right\rangle\right) \geq\left(\left\langle s p_{3}, 1\right\rangle,\left\langle\text { dist }_{2}, 0\right\rangle\right)>\left(\left\langle s p_{3}, 1\right\rangle,\left\langle\text { dist }_{2}, 1\right\rangle\right)\right\rangle
\end{aligned}
$$

\#from here ordered by budget

$$
\begin{aligned}
& \left(\left\langle s p_{15}, 0\right\rangle,\left\langle\text { dist }_{0.5}, 2\right\rangle\right) \geq\left(\left\langle s p_{15}, 2\right\rangle,\left\langle\text { dist }_{0.5}, 0\right\rangle\right) \geq\left(\left\langle s p_{8}, 0\right\rangle,\left\langle\text { dist }_{0.5}, 2\right\rangle\right) \\
& \quad \geq\left(\left\langle s p_{8}, 2\right\rangle,\left\langle\text { dist }_{0.5}, 0\right\rangle\right) \geq\left(\left\langle s p_{3}, 0\right\rangle,\left\langle\text { dist }_{0.5}, 2\right\rangle\right) \geq\left(\left\langle s p_{3}, 2\right\rangle,\left\langle\text { dist }_{0.5}, 0\right\rangle\right) \\
& \quad \geq\left(\left\langle s p_{15}, 0\right\rangle,\left\langle\text { dist }_{1}, 2\right\rangle\right) \geq\left(\left\langle s p_{15}, 2\right\rangle,\left\langle\text { dist }_{1}, 0\right\rangle\right) \geq\left(\left\langle s p_{8}, 0\right\rangle,\left\langle\text { dist }_{1}, 2\right\rangle\right) \\
& \quad \geq\left(\left\langle s p_{8}, 2\right\rangle,\left\langle\text { dist }_{1}, 0\right\rangle\right) \geq\left(\left\langle s p_{3}, 0\right\rangle,\left\langle\text { dist }_{1}, 2\right\rangle\right) \geq\left(\left\langle s p_{3}, 2\right\rangle,\left\langle\text { dist }_{1}, 0\right\rangle\right) \\
& \quad \geq\left(\left\langle s p_{15}, 0\right\rangle,\left\langle\text { dist }_{2}, 2\right\rangle\right) \geq\left(\left\langle s p_{15}, 2\right\rangle,\left\langle\text { dist }_{2}, 0\right\rangle\right) \geq\left(\left\langle s p_{8}, 0\right\rangle,\left\langle\text { dist }_{2}, 2\right\rangle\right) \\
& \quad \geq\left(\left\langle s p_{8}, 2\right\rangle,\left\langle\text { dist }_{2}, 0\right\rangle\right) \geq\left(\left\langle s p_{3}, 0\right\rangle,\left\langle\text { dist }_{2}, 2\right\rangle\right) \geq\left(\left\langle s p_{3}, 2\right\rangle,\left\langle\text { dist }_{2}, 0\right\rangle\right) \\
& \quad \geq\left(\left\langle s p_{15}, 1\right\rangle,\left\langle\text { dist }_{0.5}, 2\right\rangle\right) \geq\left(\left\langle s p_{15}, 2\right\rangle,\left\langle\text { dist }_{0.5}, 1\right\rangle\right) \geq\left(\left\langle s p_{8}, 1\right\rangle,\left\langle\text { dist }_{0.5}, 2\right\rangle\right) \\
& \quad \geq\left(\left\langle s p_{8}, 2\right\rangle,\left\langle\text { dist }_{0.5}, 1\right\rangle\right) \geq\left(\left\langle s p_{3}, 1\right\rangle,\left\langle\text { dist }_{0.5}, 2\right\rangle\right) \geq\left(\left\langle s p_{3}, 2\right\rangle,\left\langle\text { dist }_{0.5}, 1\right\rangle\right) \\
& \quad \geq\left(\left\langle s p_{15}, 1\right\rangle,\left\langle\text { dist }_{1}, 2\right\rangle\right) \geq\left(\left\langle s p_{15}, 2\right\rangle,\left\langle\text { dist }_{1}, 1\right\rangle\right) \geq\left(\left\langle s p_{8}, 1\right\rangle,\left\langle\text { dist }_{1}, 2\right\rangle\right) \\
& \quad \geq\left(\left\langle s p_{8}, 2\right\rangle,\left\langle\text { dist }_{1}, 1\right\rangle\right) \geq\left(\left\langle s p_{3}, 1\right\rangle,\left\langle\text { dist }_{1}, 2\right\rangle\right) \geq\left(\left\langle s p_{3}, 2\right\rangle,\left\langle\text { dist }_{1}, 1\right\rangle\right) \\
& \quad \geq\left(\left\langle s p_{15}, 1\right\rangle,\left\langle\text { dist }_{2}, 2\right\rangle\right) \geq\left(\left\langle s p_{15}, 2\right\rangle,\left\langle\text { dist }_{2}, 1\right\rangle\right) \geq\left(\left\langle s p_{8}, 1\right\rangle,\left\langle\text { dist }_{2}, 2\right\rangle\right) \\
& \quad \geq\left(\left\langle s p_{8}, 2\right\rangle,\left\langle\text { dist }_{2}, 1\right\rangle\right) \geq\left(\left\langle s p_{3}, 1\right\rangle,\left\langle\text { dist }_{2}, 2\right\rangle\right) \geq\left(\left\langle s p_{3}, 2\right\rangle,\left\langle\text { dist }_{2}, 1\right\rangle\right) \\
& \quad \geq\left(\left\langle s p_{15}, 2\right\rangle,\left\langle\text { dist }_{0.5}, 2\right\rangle\right) \geq\left(\left\langle s p_{8}, 2\right\rangle,\left\langle\text { dist }_{0.5}, 2\right\rangle\right) \geq\left(\left\langle s p_{3}, 2\right\rangle,\left\langle\text { dist }_{0.5}, 2\right\rangle\right) \\
& \quad \geq\left(\left\langle s p_{15}, 2\right\rangle,\left\langle\text { dist }_{1}, 2\right\rangle\right) \geq\left(\left\langle s p_{8}, 2\right\rangle,\left\langle\text { dist }_{1}, 2\right\rangle\right) \geq\left(\left\langle s p_{3}, 2\right\rangle,\left\langle\text { dist }_{1}, 2\right\rangle\right) \\
& \quad \geq\left(\left\langle s p_{15}, 2\right\rangle,\left\langle\text { dist }_{2}, 2\right\rangle\right) \geq\left(\left\langle s p_{8}, 2\right\rangle,\left\langle\text { dist }_{2}, 2\right\rangle\right) \geq\left(\left\langle s p_{3}, 2\right\rangle,\left\langle\text { dist }_{2}, 2\right\rangle\right)
\end{aligned}
$$


- BravePoor

$$
\begin{aligned}
& \left.\left\langle s p_{15}, 0\right\rangle,\left\langle\text { dist }_{0.5}, 0\right\rangle\right) \geq\left(\left\langle s p_{8}, 0\right\rangle,\left\langle\text { dist }_{0.5}, 0\right\rangle\right) \geq\left(\left\langle s p_{3}, 0\right\rangle,\left\langle\text { dist }_{0.5}, 0\right\rangle\right) \\
& \geq\left(\left\langle s p_{15}, 0\right\rangle,\left\langle\text { dist }_{1}, 0\right\rangle\right) \geq\left(\left\langle s p_{8}, 0\right\rangle,\left\langle\text { dist }_{1}, 0\right\rangle\right) \geq\left(\left\langle s p_{3}, 0\right\rangle,\left\langle\text { dist }_{1}, 0\right\rangle\right) \\
& \geq\left(\left\langle s p_{15}, 0\right\rangle,\left\langle\text { dist }_{2}, 0\right\rangle\right) \geq\left(\left\langle s p_{8}, 0\right\rangle,\left\langle\text { dist }_{2}, 0\right\rangle\right) \geq\left(\left\langle s p_{3}, 0\right\rangle,\left\langle\text { dist }_{2}, 0\right\rangle\right) \\
& >\left(\left\langle s p_{15}, 0\right\rangle,\left\langle\text { dist }_{0.5}, 1\right\rangle\right) \geq\left(\left\langle s p_{15}, 1\right\rangle,\left\langle\text { dist }_{0.5}, 0\right\rangle\right) \geq\left(\left\langle s p_{8}, 0\right\rangle,\left\langle\text { dist }_{0.5}, 1\right\rangle\right) \\
& \geq\left(\left\langle s p_{8}, 1\right\rangle,\left\langle\text { dist }_{0.5}, 0\right\rangle\right) \geq\left(\left\langle s p_{3}, 0\right\rangle,\left\langle\text { dist }_{0.5}, 1\right\rangle\right) \geq\left(\left\langle s p_{3}, 1\right\rangle,\left\langle\text { dist }_{0.5}, 0\right\rangle\right) \\
& \geq\left(\left\langle s p_{15}, 0\right\rangle,\left\langle\text { dist }_{1}, 1\right\rangle\right) \geq\left(\left\langle s p_{15}, 1\right\rangle,\left\langle\text { dist }_{1}, 0\right\rangle\right) \geq\left(\left\langle s p_{8}, 0\right\rangle,\left\langle\text { dist }_{1}, 1\right\rangle\right) \\
& \geq\left(\left\langle s p_{8}, 1\right\rangle,\left\langle\text { dist }_{1}, 0\right\rangle\right) \geq\left(\left\langle s p_{3}, 0\right\rangle,\left\langle\text { dist }_{1}, 1\right\rangle\right) \geq\left(\left\langle s p_{3}, 1\right\rangle,\left\langle\text { dist }_{1}, 0\right\rangle\right) \\
& \geq\left(\left\langle s p_{15}, 0\right\rangle,\left\langle\text { dist }_{2}, 1\right\rangle\right) \geq\left(\left\langle s p_{15}, 1\right\rangle,\left\langle\text { dist }_{2}, 0\right\rangle\right) \geq\left(\left\langle s p_{8}, 0\right\rangle,\left\langle\text { dist }_{2}, 1\right\rangle\right) \\
& \geq\left(\left\langle s p_{8}, 1\right\rangle,\left\langle\text { dist }_{2}, 0\right\rangle\right) \geq\left(\left\langle s p_{3}, 0\right\rangle,\left\langle\text { dist }_{2}, 1\right\rangle\right) \geq\left(\left\langle s p_{3}, 1\right\rangle,\left\langle\text { dist }_{2}, 0\right\rangle\right) \\
& >\left(\left\langle s p_{15}, 1\right\rangle,\left\langle\text { dist }_{0.5}, 1\right\rangle\right) \geq\left(\left\langle s p_{15}, 0\right\rangle,\left\langle\text { dist }_{0.5}, 2\right\rangle\right) \geq\left(\left\langle s p_{15}, 2\right\rangle,\left\langle\text { dist }_{0.5}, 0\right\rangle\right) \\
& \geq\left(\left\langle s p_{8}, 1\right\rangle,\left\langle\text { dist }_{0.5}, 1\right\rangle\right) \geq\left(\left\langle s p_{8}, 0\right\rangle,\left\langle\text { dist }_{0.5}, 2\right\rangle\right) \geq\left(\left\langle s p_{8}, 2\right\rangle,\left\langle\text { dist }_{0.5}, 0\right\rangle\right) \\
& \geq\left(\left\langle s p_{3}, 1\right\rangle,\left\langle\text { dist }_{0.5}, 1\right\rangle\right) \geq\left(\left\langle s p_{3}, 0\right\rangle,\left\langle\text { dist }_{0.5}, 2\right\rangle\right) \geq\left(\left\langle s p_{3}, 2\right\rangle,\left\langle\text { dist }_{0.5}, 0\right\rangle\right) \\
& \succeq\left(\left\langle s p_{15}, 1\right\rangle,\left\langle\text { dist }_{1}, 1\right\rangle\right) \geq\left(\left\langle s p_{15}, 0\right\rangle,\left\langle\text { dist }_{1}, 2\right\rangle\right) \geq\left(\left\langle s p_{15}, 2\right\rangle,\left\langle\text { dist }_{1}, 0\right\rangle\right) \\
& \succeq\left(\left\langle s p_{8}, 1\right\rangle,\left\langle\text { dist }_{1}, 1\right\rangle\right) \geq\left(\left\langle s p_{8}, 0\right\rangle,\left\langle\text { dist }_{1}, 2\right\rangle\right) \geq\left(\left\langle s p_{8}, 2\right\rangle,\left\langle\text { dist }_{1}, 0\right\rangle\right) \\
& \geq\left(\left\langle s p_{3}, 1\right\rangle,\left\langle\text { dist }_{1}, 1\right\rangle\right) \geq\left(\left\langle s p_{3}, 0\right\rangle,\left\langle\text { dist }_{1}, 2\right\rangle\right) \geq\left(\left\langle s p_{3}, 2\right\rangle,\left\langle\text { dist }_{1}, 0\right\rangle\right) \\
& \succeq\left(\left\langle s p_{15}, 1\right\rangle,\left\langle\text { dist }_{2}, 1\right\rangle\right) \geq\left(\left\langle s p_{15}, 0\right\rangle,\left\langle\text { dist }_{2}, 2\right\rangle\right) \geq\left(\left\langle s p_{15}, 2\right\rangle,\left\langle\text { dist }_{2}, 0\right\rangle\right) \\
& \geq\left(\left\langle s p_{8}, 1\right\rangle,\left\langle\text { dist }_{2}, 1\right\rangle\right) \geq\left(\left\langle s p_{8}, 0\right\rangle,\left\langle\text { dist }_{2}, 2\right\rangle\right) \geq\left(\left\langle s p_{8}, 2\right\rangle,\left\langle\text { dist }_{2}, 0\right\rangle\right) \\
& \geq\left(\left\langle s p_{3}, 1\right\rangle,\left\langle\text { dist }_{2}, 1\right\rangle\right) \geq\left(\left\langle s p_{3}, 0\right\rangle,\left\langle\text { dist }_{2}, 2\right\rangle\right) \geq\left(\left\langle s p_{3}, 2\right\rangle,\left\langle\text { dist }_{2}, 0\right\rangle\right) \\
& >\left(\left\langle s p_{15}, 1\right\rangle,\left\langle\text { dist }_{0.5}, 2\right\rangle\right) \geq\left(\left\langle s p_{15}, 2\right\rangle,\left\langle\text { dist }_{0.5}, 1\right\rangle\right) \geq\left(\left\langle s p_{8}, 1\right\rangle,\left\langle\text { dist }_{0.5}, 2\right\rangle\right) \\
& \geq\left(\left\langle s p_{8}, 2\right\rangle,\left\langle\text { dist }_{0.5}, 1\right\rangle\right) \geq\left(\left\langle s p_{3}, 1\right\rangle,\left\langle\text { dist }_{0.5}, 2\right\rangle\right) \geq\left(\left\langle s p_{3}, 2\right\rangle,\left\langle\text { dist }_{0.5}, 1\right\rangle\right) \\
& \geq\left(\left\langle s p_{15}, 1\right\rangle,\left\langle\text { dist }_{1}, 2\right\rangle\right) \geq\left(\left\langle s p_{15}, 2\right\rangle,\left\langle\text { dist }_{1}, 1\right\rangle\right) \geq\left(\left\langle s p_{8}, 1\right\rangle,\left\langle\text { dist }_{1}, 2\right\rangle\right) \\
& \succeq\left(\left\langle s p_{8}, 2\right\rangle,\left\langle\text { dist }_{1}, 1\right\rangle\right) \geq\left(\left\langle s p_{3}, 1\right\rangle,\left\langle\text { dist }_{1}, 2\right\rangle\right) \geq\left(\left\langle s p_{3}, 2\right\rangle,\left\langle\text { dist }_{1}, 1\right\rangle\right) \\
& \geq\left(\left\langle s p_{15}, 1\right\rangle,\left\langle\text { dist }_{2}, 2\right\rangle\right) \geq\left(\left\langle s p_{15}, 2\right\rangle,\left\langle\text { dist }_{2}, 1\right\rangle\right) \geq\left(\left\langle s p_{8}, 1\right\rangle,\left\langle\text { dist }_{2}, 2\right\rangle\right) \\
& \geq\left(\left\langle s p_{8}, 2\right\rangle,\left\langle\text { dist }_{2}, 1\right\rangle\right) \geq\left(\left\langle s p_{3}, 1\right\rangle,\left\langle\text { dist }_{2}, 2\right\rangle\right) \geq\left(\left\langle s p_{3}, 2\right\rangle,\left\langle\text { dist }_{2}, 1\right\rangle\right) \\
& >\left(\left\langle s p_{15}, 2\right\rangle,\left\langle\text { dist }_{0.5}, 2\right\rangle\right) \geq\left(\left\langle s p_{8}, 2\right\rangle,\left\langle\text { dist }_{0.5}, 2\right\rangle\right) \geq\left(\left\langle s p_{3}, 2\right\rangle,\left\langle\text { dist }_{0.5}, 2\right\rangle\right) \\
& \geq\left(\left\langle s p_{15}, 2\right\rangle,\left\langle\text { dist }_{1}, 2\right\rangle\right) \geq\left(\left\langle s p_{8}, 2\right\rangle,\left\langle\text { dist }_{1}, 2\right\rangle\right) \geq\left(\left\langle s p_{3}, 2\right\rangle,\left\langle\text { dist }_{1}, 2\right\rangle\right) \\
& \geq\left(\left\langle s p_{15}, 2\right\rangle,\left\langle\text { dist }_{2}, 2\right\rangle\right) \geq\left(\left\langle s p_{8}, 2\right\rangle,\left\langle\text { dist }_{2}, 2\right\rangle\right) \geq\left(\left\langle s p_{3}, 2\right\rangle,\left\langle\text { dist }_{2}, 2\right\rangle\right)
\end{aligned}
$$




\section{- Cautious}

$$
\begin{aligned}
& \left(\left\langle s p_{3}, 0\right\rangle,\left\langle\text { dist }_{2}, 0\right\rangle\right) \geq\left(\left\langle s p_{8}, 0\right\rangle,\left\langle\text { dist }_{2}, 0\right\rangle\right) \geq\left(\left\langle s p_{15}, 0\right\rangle,\left\langle\text { dist }_{2}, 0\right\rangle\right) \\
& \geq\left(\left\langle s p_{3}, 0\right\rangle,\left\langle\text { dist }_{1}, 0\right\rangle\right) \geq\left(\left\langle s p_{8}, 0\right\rangle,\left\langle\text { dist }_{1}, 0\right\rangle\right) \geq\left(\left\langle s p_{15}, 0\right\rangle,\left\langle\text { dist }_{1}, 0\right\rangle\right) \\
& \geq\left(\left\langle s p_{3}, 0\right\rangle,\left\langle\text { dist }_{0.5}, 0\right\rangle\right) \geq\left(\left\langle s p_{8}, 0\right\rangle,\left\langle\text { dist }_{0.5}, 0\right\rangle\right) \geq\left(\left\langle s p_{15}, 0\right\rangle,\left\langle\text { dist }_{0.5}, 0\right\rangle\right) \\
& >\left(\left\langle s p_{3}, 0\right\rangle,\left\langle\text { dist }_{2}, 1\right\rangle\right) \geq\left(\left\langle s p_{3}, 1\right\rangle,\left\langle\text { dist }_{2}, 0\right\rangle\right) \geq\left(\left\langle s p_{8}, 0\right\rangle,\left\langle\text { dist }_{2}, 1\right\rangle\right) \\
& \geq\left(\left\langle s p_{8}, 1\right\rangle,\left\langle\text { dist }_{2}, 0\right\rangle\right) \geq\left(\left\langle s p_{15}, 0\right\rangle,\left\langle\text { dist }_{2}, 1\right\rangle\right) \geq\left(\left\langle s p_{15}, 1\right\rangle,\left\langle\text { dist }_{2}, 0\right\rangle\right) \\
& \geq\left(\left\langle s p_{3}, 0\right\rangle,\left\langle\text { dist }_{1}, 1\right\rangle\right) \geq\left(\left\langle s p_{3}, 1\right\rangle,\left\langle\text { dist }_{1}, 0\right\rangle\right) \geq\left(\left\langle s p_{8}, 0\right\rangle,\left\langle\text { dist }_{1}, 1\right\rangle\right) \\
& \geq\left(\left\langle s p_{8}, 1\right\rangle,\left\langle\text { dist }_{1}, 0\right\rangle\right) \geq\left(\left\langle s p_{15}, 0\right\rangle,\left\langle\text { dist }_{1}, 1\right\rangle\right) \geq\left(\left\langle s p_{15}, 1\right\rangle,\left\langle\text { dist }_{1}, 0\right\rangle\right) \\
& \geq\left(\left\langle s p_{3}, 0\right\rangle,\left\langle\text { dist }_{0.5}, 1\right\rangle\right) \geq\left(\left\langle s p_{3}, 1\right\rangle,\left\langle\text { dist }_{0.5}, 0\right\rangle\right) \geq\left(\left\langle s p_{8}, 0\right\rangle,\left\langle\text { dist }_{0.5}, 1\right\rangle\right) \\
& \geq\left(\left\langle s p_{8}, 1\right\rangle,\left\langle\text { dist }_{0.5}, 0\right\rangle\right) \geq\left(\left\langle s p_{15}, 0\right\rangle,\left\langle\text { dist }_{0.5}, 1\right\rangle\right) \geq\left(\left\langle s p_{15}, 1\right\rangle,\left\langle\text { dist }_{0.5}, 0\right\rangle\right) \\
& >\left(\left\langle s p_{3}, 1\right\rangle,\left\langle\text { dist }_{2}, 1\right\rangle\right) \geq\left(\left\langle s p_{3}, 0\right\rangle,\left\langle\text { dist }_{2}, 2\right\rangle\right) \geq\left(\left\langle s p_{3}, 2\right\rangle,\left\langle\text { dist }_{2}, 0\right\rangle\right) \\
& \geq\left(\left\langle s p_{8}, 1\right\rangle,\left\langle\text { dist }_{2}, 1\right\rangle\right) \geq\left(\left\langle s p_{8}, 0\right\rangle,\left\langle\text { dist }_{2}, 2\right\rangle\right) \geq\left(\left\langle s p_{8}, 2\right\rangle,\left\langle\text { dist }_{2}, 0\right\rangle\right) \\
& \geq\left(\left\langle s p_{15}, 1\right\rangle,\left\langle\text { dist }_{2}, 1\right\rangle\right) \geq\left(\left\langle s p_{15}, 0\right\rangle,\left\langle\text { dist }_{2}, 2\right\rangle\right) \geq\left(\left\langle s p_{15}, 2\right\rangle,\left\langle\text { dist }_{2}, 0\right\rangle\right) \\
& \geq\left(\left\langle s p_{3}, 1\right\rangle,\left\langle\text { dist }_{1}, 1\right\rangle\right) \geq\left(\left\langle s p_{3}, 0\right\rangle,\left\langle\text { dist }_{1}, 2\right\rangle\right) \geq\left(\left\langle s p_{3}, 2\right\rangle,\left\langle\text { dist }_{1}, 0\right\rangle\right) \\
& \geq\left(\left\langle s p_{8}, 1\right\rangle,\left\langle\text { dist }_{1}, 1\right\rangle\right) \geq\left(\left\langle s p_{8}, 0\right\rangle,\left\langle\text { dist }_{1}, 2\right\rangle\right) \geq\left(\left\langle s p_{8}, 2\right\rangle,\left\langle\text { dist }_{1}, 0\right\rangle\right) \\
& \geq\left(\left\langle s p_{15}, 1\right\rangle,\left\langle\text { dist }_{1}, 1\right\rangle\right) \geq\left(\left\langle s p_{15}, 0\right\rangle,\left\langle\text { dist }_{1}, 2\right\rangle\right) \geq\left(\left\langle s p_{15}, 2\right\rangle,\left\langle\text { dist }_{1}, 0\right\rangle\right) \\
& \geq\left(\left\langle s p_{3}, 1\right\rangle,\left\langle\text { dist }_{0.5}, 1\right\rangle\right) \geq\left(\left\langle s p_{3}, 0\right\rangle,\left\langle\text { dist }_{0.5}, 2\right\rangle\right) \geq\left(\left\langle s p_{3}, 2\right\rangle,\left\langle\text { dist }_{0.5}, 0\right\rangle\right) \\
& \geq\left(\left\langle s p_{8}, 1\right\rangle,\left\langle\text { dist }_{0.5}, 1\right\rangle\right) \geq\left(\left\langle s p_{8}, 0\right\rangle,\left\langle\text { dist }_{0.5}, 2\right\rangle\right) \geq\left(\left\langle s p_{8}, 2\right\rangle,\left\langle\text { dist }_{0.5}, 0\right\rangle\right) \\
& \geq\left(\left\langle s p_{15}, 1\right\rangle,\left\langle\text { dist }_{0.5}, 1\right\rangle\right) \geq\left(\left\langle s p_{15}, 0\right\rangle,\left\langle\text { dist }_{0.5}, 2\right\rangle\right) \geq\left(\left\langle s p_{15}, 2\right\rangle,\left\langle\text { dist }_{0.5}, 0\right\rangle\right) \\
& >\left(\left\langle s p_{3}, 1\right\rangle,\left\langle\text { dist }_{2}, 2\right\rangle\right) \geq\left(\left\langle s p_{3}, 2\right\rangle,\left\langle\text { dist }_{2}, 1\right\rangle\right) \geq\left(\left\langle s p_{8}, 1\right\rangle,\left\langle\text { dist }_{2}, 2\right\rangle\right) \\
& \geq\left(\left\langle s p_{8}, 2\right\rangle,\left\langle\text { dist }_{2}, 1\right\rangle\right) \geq\left(\left\langle s p_{15}, 1\right\rangle,\left\langle\text { dist }_{2}, 2\right\rangle\right) \geq\left(\left\langle s p_{15}, 2\right\rangle,\left\langle\text { dist }_{2}, 1\right\rangle\right) \\
& \geq\left(\left\langle s p_{3}, 1\right\rangle,\left\langle\text { dist }_{1}, 2\right\rangle\right) \geq\left(\left\langle s p_{3}, 2\right\rangle,\left\langle\text { dist }_{1}, 1\right\rangle\right) \geq\left(\left\langle s p_{8}, 1\right\rangle,\left\langle\text { dist }_{1}, 2\right\rangle\right) \\
& \geq\left(\left\langle s p_{8}, 2\right\rangle,\left\langle\text { dist }_{1}, 1\right\rangle\right) \geq\left(\left\langle s p_{15}, 1\right\rangle,\left\langle\text { dist }_{1}, 2\right\rangle\right) \geq\left(\left\langle s p_{15}, 2\right\rangle,\left\langle\text { dist }_{1}, 1\right\rangle\right) \\
& \geq\left(\left\langle s p_{3}, 1\right\rangle,\left\langle\text { dist }_{0.5}, 2\right\rangle\right) \geq\left(\left\langle s p_{3}, 2\right\rangle,\left\langle\text { dist }_{0.5}, 1\right\rangle\right) \geq\left(\left\langle s p_{8}, 1\right\rangle,\left\langle\text { dist }_{0.5}, 2\right\rangle\right) \\
& \geq\left(\left\langle s p_{8}, 2\right\rangle,\left\langle\text { dist }_{0.5}, 1\right\rangle\right) \geq\left(\left\langle s p_{15}, 1\right\rangle,\left\langle\text { dist }_{0.5}, 2\right\rangle\right) \geq\left(\left\langle s p_{15}, 2\right\rangle,\left\langle\text { dist }_{0.5}, 1\right\rangle\right) \\
& >\left(\left\langle s p_{3}, 2\right\rangle,\left\langle\text { dist }_{2}, 2\right\rangle\right) \geq\left(\left\langle s p_{8}, 2\right\rangle,\left\langle\text { dist }_{2}, 2\right\rangle\right) \geq\left(\left\langle s p_{15}, 2\right\rangle,\left\langle\text { dist }_{2}, 2\right\rangle\right) \\
& \succeq\left(\left\langle s p_{3}, 2\right\rangle,\left\langle\text { dist }_{1}, 2\right\rangle\right) \geq\left(\left\langle s p_{8}, 2\right\rangle,\left\langle\text { dist }_{1}, 2\right\rangle\right) \geq\left(\left\langle s p_{15}, 2\right\rangle,\left\langle\text { dist }_{1}, 2\right\rangle\right) \\
& \geq\left(\left\langle s p_{3}, 2\right\rangle,\left\langle\text { dist }_{0.5}, 2\right\rangle\right) \geq\left(\left\langle s p_{8}, 2\right\rangle,\left\langle\text { dist }_{0.5}, 2\right\rangle\right) \geq\left(\left\langle s p_{15}, 2\right\rangle,\left\langle\text { dist }_{0.5}, 2\right\rangle\right)
\end{aligned}
$$

\section{References}

1. Alechina, N., Bulling, N., Dastani, M., \& Logan, B. (2015). Practical run-time norm enforcement with bounded lookahead. In Proceedings of AAMAS (pp. 443-451). https://doi.org/10.5555/2772879.27729 37.

2. Alechina, N., Dastani, M., \& Logan, B. (2013). Reasoning about normative update. In Proceedings of IJCAI (pp. 20-26). https://doi.org/10.5555/2540128.2540134.

3. Alechina, N., Dastani, M., \& Logan, B. (2014). Norm approximation for imperfect monitors. In Proceedings of AAMAS (pp. 117-124).

4. Aucher, G., Grossi, D., Herzig, A., \& Lorini, E. (2009). Dynamic context logic. In Proceedings of LORI (pp. 15-26). 
5. Baarslag, T., Kaisers, M., Gerding, E. H., Jonker, C. M., \& Gratch, J. (2017). When will negotiation agents be able to represent us? The challenges and opportunities for autonomous negotiators. In Sierra, C. (Ed.), Proceedings of the twenty-sixth international joint conference on artificial intelligence, IJCAI 2017, Melbourne, Australia, 19-25 August, 2017 (pp. 4684-4690). ijcai.org. https:// doi.org/10.24963/ijcai.2017/653.

6. Baier, J. A., \& McIlraith, S. A. (2007). On domain-independent heuristics for planning with qualitative preferences. In Logical formalizations of commonsense reasoning, papers from the 2007 AAAI spring symposium, Technical Report SS-07-05, Stanford, California, USA, 26-28 March 2007 (pp. 7-12). http://www.aaai.org/Library/Symposia/Spring/2007/ss07-05-003.php.

7. Balke, T., da Costa Pereira, C., Dignum, F., Lorini, E., Rotolo, A., Vasconcelos, W. W., et al. (2013). Norms in MAS: Definitions and related concepts. In G. Andrighetto, G. Governatori, P. Noriega, \& L. W. N. van der Torre (Eds.), Normative multi-agent systems, Dagstuhl follow-ups (Vol. 4, pp. 1-31). Wadern: Schloss Dagstuhl - Leibniz-Zentrum für Informatik. https://doi. org/10.4230/DFU.Vol4.12111.1.

8. Benabbou, N., \& Perny, P. (2017). Adaptive elicitation of preferences under uncertainty in sequential decision making problems. In Proceedings of the twenty-sixth international joint conference on artificial intelligence, IJCAI 2017, Melbourne, Australia, 19-25 August 2017 (pp. 4566-4572). https://doi.org/10.24963/ijcai.2017/637.

9. Bienvenu, M., Fritz, C., \& McIlraith, S. A. (2006). Planning with qualitative temporal preferences. In Proceedings, tenth international conference on principles of knowledge representation and reasoning, Lake District of the United Kingdom, 2-5 June 2006 (pp. 134-144). http://www.aaai.org/ Library/KR/2006/kr06-016.php.

10. Boutilier, C., Patrascu, R., Poupart, P., \& Schuurmans, D. (2006). Constraint-based optimization and utility elicitation using the minimax decision criterion. Artificial Intelligence, 170(8-9), 686713. https://doi.org/10.1016/j.artint.2006.02.003.

11. Bulling, N., \& Dastani, M. (2016). Norm-based mechanism design. Artificial Intelligence, 239, 97-142. https://doi.org/10.1016/j.artint.2016.07.001.

12. Cardoso, H. L., \& Oliveira, E. C. (2009). Adaptive deterrence sanctions in a normative framework. In Proceedings of the 2009 IEEE/WIC/ACM international conference on intelligent agent technology, IAT 2009, Milan, Italy, 15-18 September 2009 (pp. 36-43). https://doi.org/10.1109/ WI-IAT.2009.123.

13. Castelfranchi, C., Dignum, F., Jonker, C. M., \& Treur, J. (1999). Deliberative normative agents: Principles and architecture. In Intelligent agents VI, agent theories, architectures, and languages (ATAL), 6th international workshop, ATAL '99, Orlando, Florida, USA, 15-17 July 1999, Proceedings (pp. 364-378). https://doi.org/10.1007/10719619_27.

14. Chan, H., \& Darwiche, A. (2004). Sensitivity analysis in Bayesian networks: From single to multiple parameters. In UAI '04, Proceedings of the 20th conference in uncertainty in artificial intelligence, Banff, Canada, 7-11 July 2004 (pp. 67-75).

15. Chen, L., \& Pu, P. (2004). Survey of preference elicitation methods. Technical report.

16. Chopra, A. K., Dalpiaz, F., Giorgini, P., \& Mylopoulos, J. (2010). Reasoning about agents and protocols via goals and commitments. In Proceedings of the 9th international conference on autonomous agents and multiagent systems (pp. 457-464).

17. Coletti, G., Petturiti, D., \& Vantaggi, B. (2015). Rationality principles for preferences on belief functions. Kybernetika, 51(3), 486-507. https://doi.org/10.14736/kyb-2015-3-0486.

18. Cranefield, S., Meneguzzi, F., Oren, N., \& Savarimuthu, B. T. R. (2016). A Bayesian approach to norm identification. In Proceeding of ECAI (pp. 622-629). https://doi.org/10.3233/978-1-61499-672-9-622.

19. Crawford, E., \& Veloso, M. M. (2005). Learning dynamic preferences in multi-agent meeting scheduling. In A. Skowron, J. A. Barthès, L. C. Jain, R. Sun, P. Morizet-Mahoudeaux, J. Liu, \& N. Zhong (Eds.), Proceedings of the 2005 IEEE/WIC/ACM international conference on intelligent agent technology, Compiegne, France, 19-22 September 2005 (pp. 487-490). IEEE Computer Society. https://doi.org/10.1109/IAT.2005.94.

20. Dasgupta, A., \& Ghose, A. K. (2010). BDI agents with objectives and preferences. In Declarative agent languages and technologies VIII-8th international workshop, DALT 2010, Toronto, Canada, 10 May 2010, Revised, Selected and Invited Papers (pp. 22-39). https://doi.org/10.1007/978-3642-20715-0_2.

21. Dastani, M., Dignum, F., \& Meyer, J. C. (2003). Autonomy and agent deliberation. In M. Nickles, M. Rovatsos, \& G. Weiß (Eds.), Agents and computational autonomy-Potential, risks, and solutionsPostproceedings of the 1st international workshop on computational autonomy-Potential, risks, solutions (AUTONOMY 2003), held at the 2nd international joint conference on autonomous agents and 
multi-agent systems (AAMAS 2003), 14 July 2003, Melbourne, Australia, Lecture Notes in Computer Science (Vol. 2969, pp. 114-127). Springer. https://doi.org/10.1007/978-3-540-25928-2_10.

22. Dastani, M., Grossi, D., Meyer, J. C., \& Tinnemeier, N. A. M. (2009). Normative multi-agent programs and their logics. In Normative multi-agent systems. http://drops.dagstuhl.de/opus/volltexte/2009/1907/.

23. Dell'Anna, D., Dastani, M., \& Dalpiaz, F. (2018). Runtime norm revision using Bayesian networks. In Proceedings of the 21st international conference on principles and practice of multi-agent systems (PRIMA2018).

24. Dell'Anna, D., Dastani, M., \& Dalpiaz, F. (2019). Runtime revision of norms and sanctions based on agent preferences. In Proceedings of the 18th international conference on autonomous agents and multiagent systems, AAMAS '19, Montreal, QC, Canada, 13-17 May 2019 (pp. 1609-1617). https:// doi.org/10.5555/3306127.3331881.

25. Dell'Anna, D., Dastani, M., \& Dalpiaz, F. (2020). Runtime revision of sanctions in normative multiagent systems-Supplementary Material-SASS. https://doi.org/10.5281/zenodo.3712045.

26. Evans, O., Stuhlmüller, A., \& Goodman, N. D. (2016). Learning the preferences of ignorant, inconsistent agents. In D. Schuurmans \& M. P. Wellman (Eds.), Proceedings of the thirtieth AAAI conference on artificial intelligence, 12-17 February 2016, Phoenix, Arizona, USA (pp. 323-329). AAAI Press. http://www.aaai.org/ocs/index.php/AAAI/AAAI16/paper/view/12476.

27. Fishburn, P. C. (1975). Axioms for lexicographic preferences. The Review of Economic Studies, 42(3), 415-419.

28. Governatori, G., \& Rotolo, A. (2010). Changing legal systems: Legal abrogations and annulments in defeasible logic. Logic Journal of the IGPL, 18(1), 157-194. https://doi.org/10.1093/jigpal/jzp075.

29. Hausman, D. M. (2011). Preference, value, choice, and welfare. Cambridge: Cambridge University Press.

30. Jiang, J., Aldewereld, H., Dignum, V., \& Tan, Y. (2012). Norm contextualization. In Coordination, organizations, institutions, and norms in agent systems VIII-14th international workshop, COIN 2012, Held Co-located with AAMAS 2012, Valencia, Spain, 5 June 2012, Revised Selected Papers (pp. 141-157). https://doi.org/10.1007/978-3-642-37756-3_9.

31. Jiang, J., Thangarajah, J., Aldewereld, H., \& Dignum, V. (2014). Reasoning with agent preferences in normative multi-agent systems. In International conference on autonomous agents and multi-agent systems, AAMAS '14, Paris, France, 5-9 May 2014 (pp. 1373-1374). https://doi.org/10.5555/26157 31.2617479 .

32. Knobbout, M., \& Dastani, M. (2012). Reasoning under compliance assumptions in normative multiagent systems. In Proceedings of AAMAS (pp. 331-340).

33. Knobbout, M., Dastani, M., \& Meyer, J. C. (2014). Reasoning about dynamic normative systems. In Proceedings of JELIA (pp. 628-636). https://doi.org/10.1007/978-3-319-11558-0_46.

34. Knobbout, M., Dastani, M., \& Meyer, J. C. (2016). A dynamic logic of norm change. In Proceedings of ECAI (pp. 886-894). https://doi.org/10.3233/978-1-61499-672-9-886.

35. Krajzewicz, D., Erdmann, J., Behrisch, M., \& Bieker, L. (2012). Recent development and applications of SUMO-Simulation of Urban MObility. International Journal on Advances in Systems and Measurements, 5(3\&4), 128-138.

36. Kwisthout, J. (2011). Most probable explanations in bayesian networks: Complexity and tractability. International Journal of Approximate Reasoning, 52(9), 1452-1469. https://doi.org/10.1016/j. ijar.2011.08.003.

37. Mas-Colell, A., Whinston, M. D., Green, J. R., et al. (1995). Microeconomic theory (Vol. 1). New York: Oxford University Press.

38. Miralles, J. C., López-Sánchez, M., Salamó, M., Avila, P., \& Rodríguez-Aguilar, J. A. (2013). Robust regulation adaptation in multi-agent systems. TAAS, 8(3), 13:1-13:27. https://doi.org/10.1145/25173 28.

39. Mostafa, S. A., Ahmad, M. S., \& Mustapha, A. (2019). Adjustable autonomy: A systematic literature review. Artificial Intelligence Review, 51(2), 149-186. https://doi.org/10.1007/s10462-017-9560-8.

40. Natarajan, S., \& Tadepalli, P. (2005). Dynamic preferences in multi-criteria reinforcement learning. In L. D. Raedt \& S. Wrobel (Eds.), Machine learning, proceedings of the twenty-second international conference (ICML 2005), Bonn, Germany, 7-11 August 2005, ACM International Conference Proceeding Series (Vol. 119, pp. 601-608). ACM. https://doi.org/10.1145/1102351.1102427.

41. Padgham, L., \& Singh, D. (2013). Situational preferences for BDI plans. In International conference on autonomous agents and multi-agent systems, AAMAS '13, Saint Paul, MN, USA, 6-10 May 2013 (pp. 1013-1020). https://doi.org/10.5555/2484920.2485080.

42. Russell, S. J., \& Norvig, P. (2010). Artificial intelligence-A modern approach (3 internat. ed.). London: Pearson Education. 
43. Schmid, S., Gerostathopoulos, I., Prehofer, C., \& Bures, T. (2017). Self-adaptation based on big data analytics: A model problem and tool. In Proceedings of SEAMS (pp. 102-108). https://doi.org/10.1109/ SEAMS.2017.20.

44. Serral, E., Sernani, P., Dragoni, A. F., \& alpiaz, F., (2017). Contextual requirements prioritization and its application to smart homes. In A. Braun, R. Wichert, \& A. Maña (Eds.), Ambient intelligence-13th European conference, AmI 2017, Malaga, Spain, 26-28 April 2017, Proceedings, Lecture Notes in Computer Science (Vol. 10217, pp. 94-109). https://doi.org/10.1007/978-3-319-56997-0_7.

45. Snoek, J., Larochelle, H., \& Adams, R. P. (2012). Practical Bayesian optimization of machine learning algorithms. In Advances in neural information processing systems 25: 26th annual conference on neural information processing systems 2012. Proceedings of a meeting held 3-6 December 2012, Lake Tahoe, Nevada, USA (pp. 2960-2968). http://papers.nips.cc/paper/4522-practical-bayesian-optimizati on-of-machine-learning-algorithms.

46. Sugiyama, Y., Fukui, M., Kikuchi, M., Hasebe, K., Nakayama, A., Nishinari, K., et al. (2008). Traffic jams without bottlenecks-Experimental evidence for the physical mechanism of the formation of a jam. New Journal of Physics, 10(3), 033001.

47. Testerink, B., Dastani, M., \& Bulling, N. (2016). Distributed controllers for norm enforcement. In Proceedings of ECAI (pp. 751-759). https://doi.org/10.3233/978-1-61499-672-9-751.

48. Tumer, K., Welch, Z. T., \& Agogino, A. (2008). Aligning social welfare and agent preferences to alleviate traffic congestion. In Proceedings of the 7th international joint conference on autonomous agents and multiagent systems (Vol. 2, pp. 655-662). International Foundation for Autonomous Agents and Multiagent Systems

49. van Benthem, J., \& Liu, F. (2007). Dynamic logic of preference upgrade. Journal of Applied NonClassical Logics, 17(2), 157-182. https://doi.org/10.3166/jancl.17.157-182.

50. van Riemsdijk, M. B., Hindriks, K. V., \& Jonker, C. M. (2009). Programming organization-aware agents. In Engineering societies in the agents world X, 10th international workshop, ESAW 2009, Utrecht, The Netherlands, 18-20 November 2009. Proceedings (pp. 98-112). https://doi.org/10.1007/9783-642-10203-5_9.

51. Vasconcelos, W. W., Kollingbaum, M. J., \& Norman, T. J. (2009). Normative conflict resolution in multi-agent systems. Autonomous Agents and Multi-Agent Systems, 19(2), 124-152. https://doi. org/10.1007/s10458-008-9070-9.

52. Vázquez-Salceda, J., Aldewereld, H., Grossi, D., \& Dignum, F. (2008). From human regulations to regulated software agents' behavior. Artificial Intelligence and Law, 16(1), 73-87. https://doi.org/10.1007/ s10506-007-9057-X.

53. Visser, S., Thangarajah, J., Harland, J., \& Dignum, F. (2016). Preference-based reasoning in BDI agent systems. Autonomous Agents and Multi-Agent Systems, 30(2), 291-330. https://doi.org/10.1007/s1045 8-015-9288-2.

54. Wang, H., Rish, I., \& Ma, S. (2002). Using sensitivity analysis for selective parameter update in Bayesian network learning. Palo Alto, CA: Association for the Advancement of Artificial Intelligence (AAAI).

55. Wellman, M. P. (1990). Fundamental concepts of qualitative probabilistic networks. Artificial Intelligence, 44(3), 257-303. https://doi.org/10.1016/0004-3702(90)90026-V.

56. Wellman, M. P., \& Henrion, M. (1993). Explaining 'explaining away'. IEEE Transactions on Pattern Analysis and Machine Intelligence, 15(3), 287-292. https://doi.org/10.1109/34.204911.

57. Wooldridge, M. J. (2009). An Introduction to MultiAgent Systems (2nd ed.). Hoboken, NJ: Wiley.

58. Ziebart, B. D., Dey, A. K., \& Bagnell, J. A. (2008). Fast planning for dynamic preferences. In J. Rintanen, B. Nebel, J. C. Beck, \& E. A. Hansen (Eds.), Proceedings of the eighteenth international conference on automated planning and scheduling, ICAPS 2008, Sydney, Australia, 14-18 September 2008 (pp. 412-419). AAAI. http://www.aaai.org/Library/ICAPS/2008/icaps08-051.php.

Publisher's Note Springer Nature remains neutral with regard to jurisdictional claims in published maps and institutional affiliations. 